\title{
Population Dynamics Approach FOR \\ Resource Allocation Problems
}

by

\author{
Ashkan Pashaie
}

A thesis submitted in conformity with the requirements for the degree of Master of Applied Science Graduate Department of Electrical and Computer Engineering University of Toronto

(C) Copyright 2014 by Ashkan Pashaie 


\author{
Abstract \\ Population Dynamics Approach for Resource Allocation Problems \\ Ashkan Pashaie \\ Master of Applied Science \\ Graduate Department of Electrical and Computer Engineering \\ University of Toronto \\ 2014
}

Resource allocation management has become a challenging problem in many aspects all around the world and can be more complicated by high production costs and scarcity of the resources. In this thesis, a water distribution system (WDS), as an example of resource distribution problems, has been investigated. To achieve an adequate level of service to satisfy the demands, the flow of water should be controlled. Here, we utilize a novel game-theoretic approach along with a convex-optimization-based controller to keep the WDS under control. The feedback interconnection of the WDS and controllers reaches an asymptotically stable equilibrium point where its stability analysis uses passivity concepts and the Lyapunov stability theorem for the closed-loop system. Furthermore, an improvement has been made to controllers to make the system robust to reject a certain type of disturbance and to converge to desired equilibrium points in the presence of disturbances. 


\section{Acknowledgements}

I would like to express my sincere gratitude to my Master supervisors, Profs. Lacra Pavel and Christopher J. Damaren for their continued assistance, guidance and dedication for the duration of my Master program. Our general and technical discussions have been fundamental to my growth as a researcher in the Systems Control Group at the University of Toronto. They have always been willing to share their perspectives, insights and methodologies in several aspects of the project in a thorough manner. Finally, they have spent significant time to thoroughly review my thesis and make useful suggestions.

Furthermore, I would like to acknowledge my friends whose support is much appreciated. Last but not least, I would like to thank my family and my parents for their continued support during my university career. 


\section{Contents}

$\begin{array}{lll}1 & \text { Introduction } & 1\end{array}$

1.1 Motivating Example $\ldots \ldots \ldots \ldots \ldots$. . . . . . . . . . . . . . . 2

1.2 Literature Review . . . . . . . . . . . . . . . . . . . . . . . . . 4

1.3 Thesis Organization. . . . . . . . . . . . . . . . . . . . . . . 6

1.4 Statement of Contributions . . . . . . . . . . . . . . . 8

\begin{tabular}{lll}
\hline I & Preliminaries/Background & 11
\end{tabular}

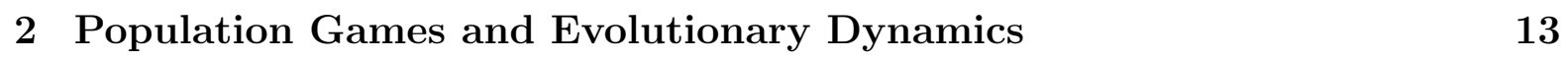

$2.1 \quad$ Population Games $\ldots \ldots \ldots$

2.1 .1 Populations, Strategies and States . . . . . . . . . . . . . . . 13

$2.1 .2 \quad$ Payoffs, Best Responses, Nash Equilibria . . . . . . . . . . . 14

$2.2 \quad$ Evolutionary Dynamics $\ldots \ldots \ldots \ldots$

$2.2 .1 \quad$ Revision Protocols, Mean Dynamics . . . . . . . . . . . . 17

$2.2 .2 \quad$ Positive Correlation, Nash Stationary . . . . . . . . . . 20

2.2 .3 Classes of Evolutionary Dynamics . . . . . . . . . . . . . 21

2.2 .4 Connection of Games and Evolutionary Dynamics . . . . . . . . 29

$\begin{array}{lll}3 & \text { Stability and Passivity } & 33\end{array}$

$3.1 \quad$ Passivity and Lyapunov Stability $\ldots \ldots \ldots$

3.2 Feedback Connection . . . . . . . . . . . . . . . . . 36 
3.3 Positive Real Transfer Functions . . . . . . . . . . . . . . . . . . . . . . . . . . 39

3.4 Summary $\ldots \ldots \ldots \ldots \ldots$

II Modeling and Stability Analysis 43

4 Water Distribution System - Modeling/Control Problem 45

4.1 Preview - Motivation . . . . . . . . . . . . . . . . . . . . . . . 45

$4.2 \quad$ Water Distribution System; $N$-Tank System … . . . . . . . . . . 49

4.3 System Controllers $\ldots \ldots \ldots \ldots \ldots$

$4.3 .1 \quad$ Individual Input Flow Control . . . . . . . . . . . . . . . 51

4.3 .2 Total Input Flow Control $\ldots \ldots \ldots \ldots$

$4.4 \quad$ Feedback Interconnection - Standard Notation . . . . . . . . . . . . . . 54

$\begin{array}{lll}5 & \text { Stability Analysis } & 61\end{array}$

$5.1 \quad$ The Replicator Dynamic . . . . . . . . . . . . . . . . . . . . . . . . 61

$5.1 .1 \quad$ Passivity of the Interconnected System . . . . . . . . . . . . 62

5.2 Convergence to the Interior $N E \ldots \ldots \ldots$

5.2 .1 The BNN Dynamic . . . . . . . . . . . . . . . . . . . 69

5.2 .2 The Smith Dynamic $\ldots \ldots \ldots \ldots \ldots \ldots$

5.3 Summary $\ldots \ldots \ldots \ldots \ldots \ldots$

\begin{tabular}{lll}
\hline 6 & Disturbance Rejection & 77
\end{tabular}

$6.1 \quad$ Modeling . . . . . . . . . . . . . . . . . . . . . . . . . . . . . . . . . . . . . . . . . 77

6.2 Analysis $\ldots \ldots \ldots \ldots \ldots \ldots$

6.3 Summary $\ldots \ldots \ldots \ldots \ldots$

$\begin{array}{lll}7 & \text { Global Convergence; Generalization } & 83\end{array}$

7.1 Globally Asymptotic Stability . . . . . . . . . . . . . . . . . . . 83

7.2 Affine Games . . . . . . . . . . . . . . . . . . . . . . . . 90 
7.3 Summary $\ldots \ldots \ldots \ldots$

III Simulation and Conclusion $\quad 95$

\begin{tabular}{lll}
\hline & Simulation Results & 97
\end{tabular}

$8.1 \quad$ The Replicator, BNN and Smith Dynamics . . . . . . . . . . . . . 97

8.2 Convergence to the Interior $N E \ldots \ldots \ldots \ldots \ldots$

8.3 Disturbance Rejection . . . . . . . . . . . . . . . . . . . . . . 103

$\begin{array}{lll}9 \text { Conclusions } & 109\end{array}$

$9.1 \quad$ Concluding Remarks . . . . . . . . . . . . . . . . . . . . . . . 109

$9.2 \quad$ Future Work . . . . . . . . . . . . . . . . . . . . . . . . . . . . . . . . 111

\begin{tabular}{lll}
\hline IV Appendices & 113 \\
\hline
\end{tabular}

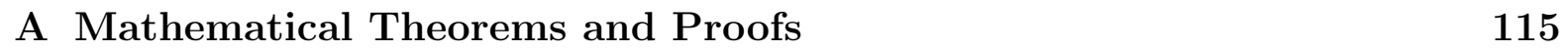

A.1 Positive Definite Symmetric Matrices . . . . . . . . . . . . . . . . . . 115

A.2 Proof of Theorem $5.3 \ldots \ldots \ldots$

A.3 Domain of Attraction . . . . . . . . . . . . . . . . . . . . . . 118

\begin{tabular}{ll}
\hline B Convex Optimization & 123
\end{tabular}

B.1 Gradient Method . . . . . . . . . . . . . . . . . . . . . . . . . . . . . . 123

\begin{tabular}{ll}
\hline Bibliography & 125
\end{tabular} 


\section{List of Figures}

1.1 Water distribution system example. . . . . . . . . . . . . . . . . . . . . 3

$1.2 \quad N$-tank system. $\ldots \ldots \ldots \ldots \ldots$

$2.1 \quad$ Population games as mappings from population state to payoffs. . . . . 14

$2.2 \quad$ Evolutionary dynamics as mappings from payoffs to population state. . 19

$2.3 \quad$ Connection of population games and evolutionary dynamics. . . . . . . 30

2.4 Negative feedback interconnection of two systems. . . . . . . . . . . . . 30

$3.1 \quad$ Negative feedback interconnection of the systems in the form of (3.1). . 36

$3.2 \quad$ Feedback interconnection of three systems of form (3.1). . . . . . . . . . 38

$4.1 \quad$ Connection of dynamical system of games and evolutionary dynamics. . 47

4.2 Water Distribution System. . . . . . . . . . . . . . . . . . . . . . . . 49

$4.3 \quad$ Feedback interconnection of the $N$-tank system with the evolutionary dynamic and the total input flow controllers. . . . . . . . . . . . . . . 55

$5.1 \quad$ Feedback interconnection of the concatenated system $\Sigma_{1}^{\prime}$ and $\Sigma_{2}$. . . . 65

$6.1 \quad$ Added integrator to the feedback interconnected system. . . . . . . . . . 78

6.2 Integrator and the gradient controller, $\Sigma_{3}^{\prime} . \ldots \ldots \ldots$

$7.1 \quad$ Negative feedback interconnection of the game and the evolutionary dynamic. . . . . . . . . . . . . . . . . . . . 84 
8.1 Tank system and the Replicator Dynamic, with initial condition 1. . . . . 99

8.2 Tank system and the BNN dynamic, with initial condition 1. . . . . . . . 99

8.3 Tank system and the Smith dynamic, with initial condition 1. . . . . . . 100

8.4 Tank system and the Replicator Dynamic, with initial condition 2. . . . . 101

8.5 Tank system and the BNN dynamic, with initial condition 2. . . . . . . . 102

8.6 Tank system and the Smith dynamic, with initial condition 2. . . . . . . 102

8.7 Step-like disturbance. . . . . . . . . . . . . . . . . . . . . . . . . . . 103

8.8 Tank system and the BNN dynamic. . . . . . . . . . . . . . . . . . . . . 104

8.9 Total input flow of the tank system in connection with the BNN dynamic. 105

8.10 Tank system and the Smith dynamic. . . . . . . . . . . . . . . . . . . . 106

8.11 Stochastic rectangular disturbance pulses. . . . . . . . . . . . . . . . . . 107

8.12 Tank system and the Smith dynamic. . . . . . . . . . . . . . . . . . . . . 108 


\section{List of Tables}

2.1 Classes of evolutionary dynamics and their properties. . . . . . . . . . 29

8.1 Tank system and total input flow controller parameters. . . . . . . . . . 98

$8.2 \quad$ System initial condition $1 . \ldots \ldots \ldots$ 


\title{
List of Symbols and Acronyms
}

\author{
Sets, Fields, Vectors and Matrices: \\ $\mathbb{R} \quad$ Field of real numbers \\ $\mathbb{R}^{n} \quad$ The set of $n$ tuples of the real numbers \\ $\mathbb{R}^{n \times m} \quad$ The set of $n \times m$ matrices with real elements \\ $\mathbb{R}_{+} \quad$ The set of non-negative real numbers \\ $x \in \mathcal{K} \quad x$ is an element of the set $\mathcal{K}$ \\ $x \notin \mathcal{K} \quad x$ is not an element of the set $\mathcal{K}$ \\ $\mathcal{K}_{1} \subset \mathcal{K}_{2} \quad$ The set $\mathcal{K}_{1}$ is contained in the set $\mathcal{K}_{2}$ \\ $\mathcal{K}_{1} \subseteq \mathcal{K}_{2} \quad$ The set $\mathcal{K}_{1}$ is contained in or is equal to the set $\mathcal{K}_{2}$ \\ $F: \mathcal{D}_{1} \rightarrow \mathcal{D}_{2} \quad F$ maps a vector in domain $\mathcal{D}_{1}$ to a vector in co-domain $\mathcal{D}_{2}$ \\ $A^{T} \quad$ Transpose of $A$ \\ $A^{-1} \quad$ Inverse of the square matrix $A$ \\ $I_{N} \quad$ The $N \times N$ identity matrix \\ II The square matrix of ones \\ 1 The vector of ones
}




$\begin{array}{ll}\|x\|_{2} \text { or }\|x\| & \text { The Euclidean norm of the vector } x \\ \|x\|_{p} & \text { The } p \text {-norm of the vector } x \\ \|A\|_{p} & \text { The induced } p \text {-norm of the matrix } A \\ x \cdot y=x^{T} y & \text { The inner product of the vectors } x \text { and } y\end{array}$

\section{Derivatives:}

$d f / d x \quad$ Derivative of $f$ with respect to $x$

$\partial f / \partial x \quad$ Partial derivative of $f$ with respect to $x$

$\nabla_{x} f \quad$ If $x$ is a vector and $f$ is a differentiable function, this is the directional derivative of $f$ in the direction of $x$.

\section{Acronyms:}

$\begin{array}{ll}\text { WDS } & \text { Water Distribution System } \\ \text { BNN } & \text { Brown-Von Neumann-Nash } \\ \text { EPT } & \text { Excess Payoff Target } \\ \text { LTI } & \text { Ninear Time Invariant } \\ \text { NE } & \text { Positive Correlation } \\ \text { PC } & \text { Nash Stationary } \\ \text { NS } & \text { Single Input Single Output }\end{array}$




$\begin{array}{ll}\text { D.o.A } & \text { Domain of Attraction } \\ \text { RHS } & \text { Right Hand Side } \\ \text { SNR } & \text { Signal to Noise Ratio } \\ \text { OLHP } & \text { Open Left Half Plane } \\ \text { ODE } & \text { Ordinary Differential Equation }\end{array}$




\section{Chapter 1}

\section{Introduction}

Resource allocation management is a subject of growing concern all around the world. Resource distribution as a part of the resource management is used to assign certain amounts of resources to consumers in an economic and efficient way. However, the scarcity of the resources, environmental protection considerations, sustainability and providing satisfactory level of service are some of the existing constraints that make the resource allocation problem a big challenge to deal with.

Having plans and strategies for using scarce resources to achieve appropriate and desirable outcomes of the systems under study while the efficiency and economic considerations are fulfilled is one of the difficulties of this area that inspired us to utilize a novel approach to solve the problem of the resource distribution in this thesis.

The allocation problem can take different forms with respect to type of demands and resources in use. Labor, energy, time, fuel, material, etc. are some examples of the abundant number of resources that need to be controlled. In these problems, even a simple project can turn into a complicated challenging problem to find an efficient solution approach.

However, despite the difference in types of resource allocation, distribution problems have a common goal in their solution approaches that is cost minimization. In fact, 
various proposed methods seek an optimal point where the production, which is the purpose of systems, is maximized while the consumption of resources is minimized. One of the most well-known examples that can be found in literature is the application in wireless communication where the transmitters are supposed to send and receive data to each other and to one or multiple base stations. In this field, it has been tried to supply the transmitters with the minimum power while the signal-to-noise ratio (SNR) remains the maximum possible.

In these problems, diverse methods of convex optimizations are applicable to achieve the cost minimization goal. Usually by defining a cost function to be minimized or maximizing a utility function that is defined based on the system variables, the problem can be partially solved. Some examples of these methods are presented in Section 1.2 .

However, in this thesis, we utilize an evolutionary game-theoretic strategy where multiple strategies are defined based on the variables of the system and a large number of agents are set to play the strategies. In this configuration, not only the system is stabilized and a desirable behavior is achieved, but also optimal equilibrium points of the system where all strategies get maximum utility can be reached. In the next section, a motivating example to this work as well as a game-theoretic solution approach is presented.

\subsection{Motivating Example}

Management of a water distribution network has been one of the most important issues of concern in big cities. Fast growing population, scarcity of drinkable water, high costs of water treatments, etc. are the difficulties that experts in the field of water supply are challenged by. Changes in water demand patterns, quality of service and different types of water consumption such as domestic, industrial, agricultural and etc. have resulted 


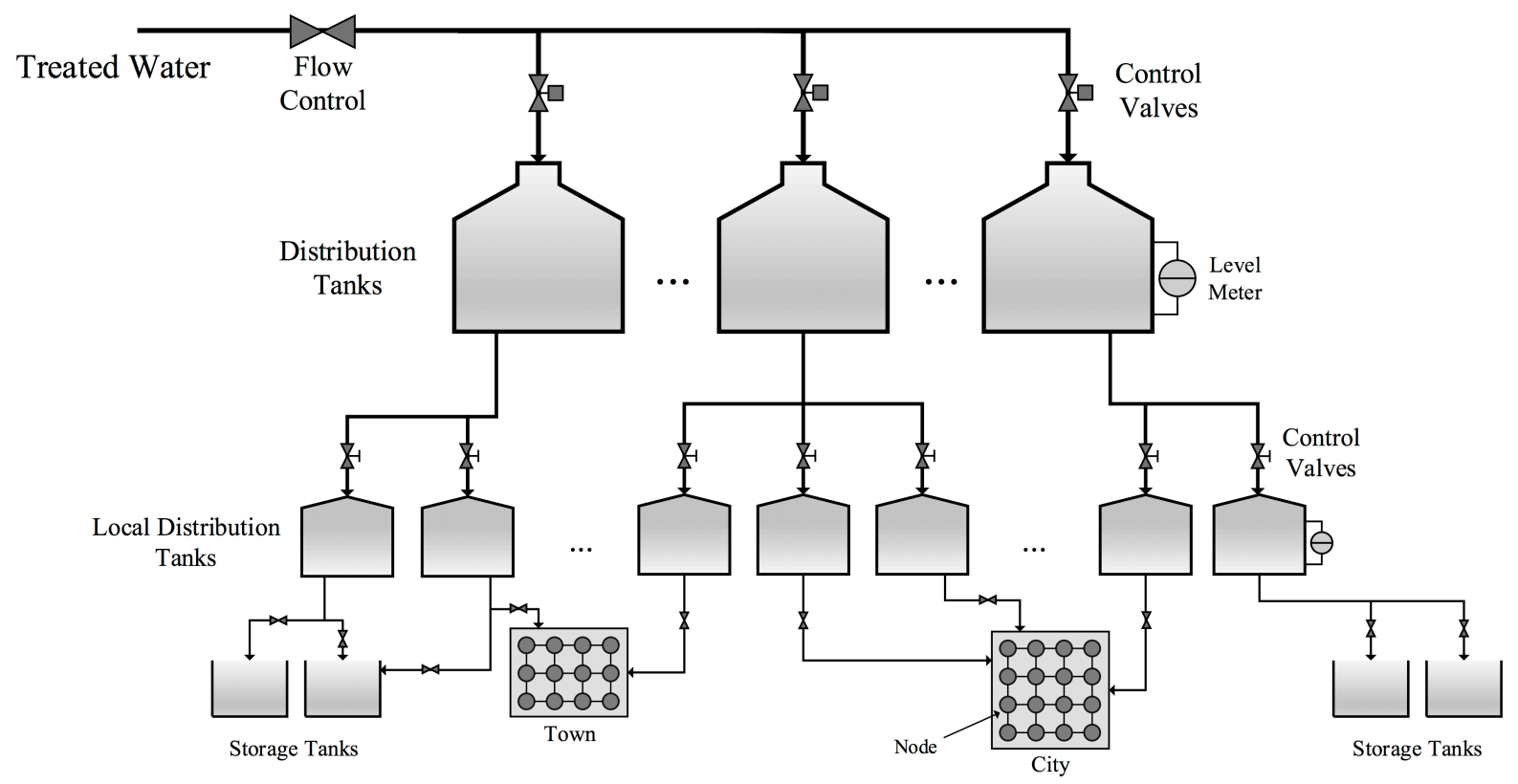

Figure 1.1: Water distribution system example.

in research for finding and establishing the most suitable and reliable system that can adapt to several kinds of demands.

Figure 1.1 illustrates a simple network of a Water Distribution System (WDS) that has different layers of distribution tanks to supply the consumers which consist of domestic in towns and cities and also industrial usages. Finding a system that can meet the demands, which are explained above, is the objective of this thesis.

As a straightforward example of a WDS, consider the $N$-tank system, shown in Figure 1.2, where $N$ tanks are utilized to supply $N$ consumers. In this system, each tank has a separate input and output. To supply the tanks with water, we need to split a total input flow of water, which is considered as the resource here, between the tanks inputs. Also to meet the demands, we are supposed to measure the output of the tanks. As a high quality service, customers expect a continuous flow of water with appropriate pressure at the corresponding node. Therefore, we need to control the flow of water to and from each tank to satisfy each consumer with the supplied water, which means having appropriate volume of water in the tanks. 


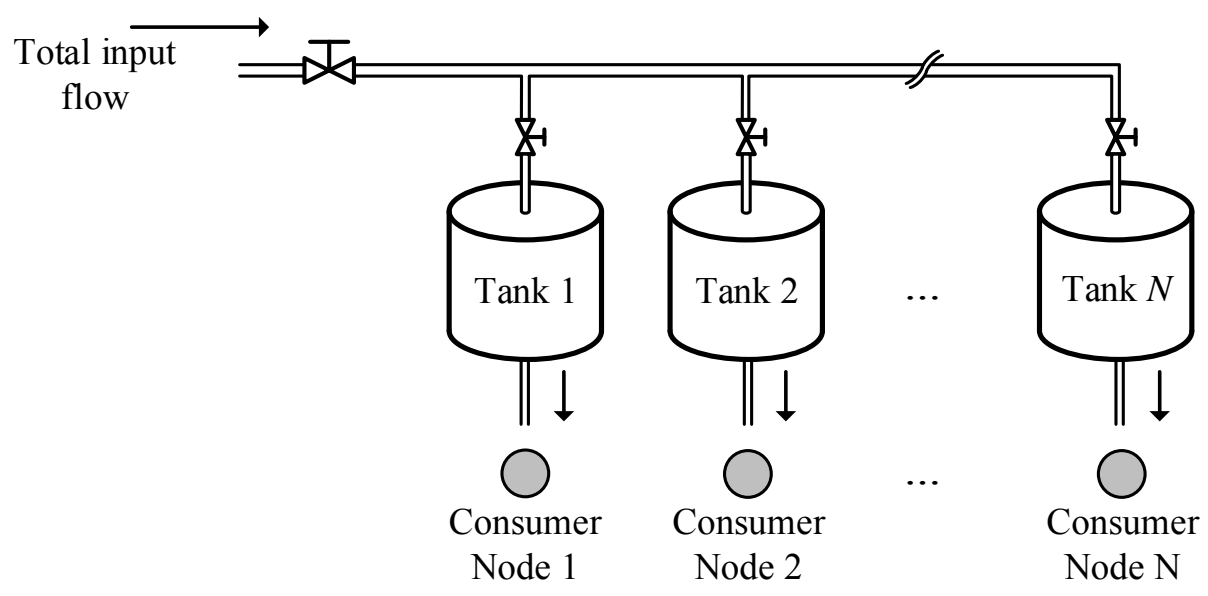

Figure 1.2: $N$-tank system.

In this thesis, we will control the $N$-tank system by using an evolutionary gametheoretic approach and will define a game based on our system. In population games, as one of the categories of games in the concept of game theory, there are a finite number of strategies being played by a large number of agents/players. By defining a payoff for each strategy, agents can decide which strategy to select and play. So to control the $N$-tank system via the population games, we have to set up our game based on the WDS. If we consider each tank as a strategy and introduce a payoff associated with the selected tank, we can assume that the artificial players are selecting between these strategies. By playing each tank, it is assumed that a certain amount of the total input flow is allocated to fill the corresponding tank. Thus, by choosing each tank as a strategy selection by the agents, the $N$-tank system gets filled with water. Therefore, if we make the agents fill the tanks in our desirable way, the goal of the system could be achieved.

\subsection{Literature Review}

Recently, there has been considerable interest in designing optimal control strategies in source allocation problems. High level of demands as well as expectations of good quality service, scarcity of the resources and cost of energy generation have led researchers around the world to investigate the allocation problems in several fields. In wireless com- 
munication networks, authors of many articles tried to minimize the power consumption while customers are satisfied with service quality which could be defined based on signalto-noise ratio (SNR) [1, 2, 3, 4, 13, 37, 41, 42]. In these articles, it is assumed that the power is correlated with the SNR in the way that by increasing power, range will be extended but the noise level will be higher too [22, 26, 27, 28, 31]. Therefore, by defining cost functions with respect to the power and SNR and utilizing a game theoretic approach, the problem of power distribution is solved. Another approach that could be found in the literature, benefits from convex optimization concepts [9] and linear and non-linear programming [7, 23]. In these works, one of the most well-known methods, called the gradient descent algorithm, is used to optimize power consumption [14, 16]. In these articles, usually not all dynamics of the system under study are considered in the calculations, but partial dynamics or the functions that are linked to the power and service quality are characterized. Generally, in these problems, game-theoretic solutions are affected by players interaction and strategy picking based on others' choices.

On the other hand, in other areas such as [29, 30] where the objective is to control the temperature of a building using population games, the authors paid attention to whole dynamics of systems, i.e., the process of how the system under study evolves has been brought into attention. In these articles, a population game is employed not only to optimize the system, but also to stabilize it. The author has provided some results that confirm the stability of the systems and also prove the efficiency of the model in comparison to the controllers that are commonly employed in industry. Typical applications in this area involve a very basic on-off control approach, which is not only efficient but also harmful for the machinery and devices that supply the resources to the systems. Other examples of utilizing a game-theoretic approach for optimization are found in [33, 34] that are the inspiration for this thesis. In these papers, the authors have investigated water distribution systems [6].

By reviewing the literature, we found that not much work has been done on the area of 
the water distribution system. One approach that has been found uses cost minimization of materials such as pipe, valves, tank and etc. [8, 11, 38, 43]. The second approach that has been used is based on minimizing water production cost, so the consumption of water is important in this case [5, 12]. However, in the above mentioned works, no mathematical proofs have been provided. Therefore, the approach of population games with the analytical study, which is presented in [34] is a big step in characterizing, stabilizing and optimizing the water distribution systems. The authors of this work used the Replicator Dynamic as the centralized controller of the tank system to stabilize and make the system converge to the desirable equilibrium points. The Replicator Dynamic is one of the classes of evolutionary dynamics of games that was first introduced in [39] where the author was inspired by evolution of biological system. Also the authors used some stability analysis tools to prove the stability of these systems.

A significant point of this optimization approach that distinguishes between this method and a regulation problem is that by tuning the parameters of the controller

properly, the desired equilibrium points are found through an online optimization by the system itself. In fact, the outputs are not regulated to converge to the desired values.

The evolutionary dynamics that represent dynamical models of population games, monitor the evolution of games with respect to time. Therefore, controlling a dynamical system such as WDS with evolutionary dynamics let us to observe the process under which the systems evolve. Different classes of evolutionary dynamics as well as detailed definitions and analysis can be found in [19, 20, 21, 32, 35, 36, 40. In terms of the stability analysis of the evolutionary dynamics, the authors in $[17,18,19,20,21,32,34,35,36$, used the passivity concepts and Lyapunov stability theorem [25].

\subsection{Thesis Organization}

The body of this thesis is structured into three parts. 
1. Part I; Preliminaries/Background.

We provide required preliminaries for this thesis. This part includes two chapters.

- Chapter 2: Population Games and Evolutionary Dynamics.

This chapter reviews population games, definitions of population and Nash equilibrium points. Also evolutionary dynamics with different classes and properties as well as their dynamical model and the definition of revision protocols are introduced.

- Chapter 3: Stability and Passivity.

In this chapter, the definition of passivity, the Lyapunov stability theorem and the properties of passive systems in negative feedback interconnections are reviewed. Complementary lemmas and the definition of positive real transfer functions are provided.

2. Part II; Modeling and Stability Analysis.

This part consists of modeling and stability analysis of the system under study. Four chapters are listed in this part.

- Chapter 4: Water Distribution System - Modeling/Control Problem.

We introduce the model of the system under study in detail. We introduce a dynamical model of the $N$-tank system as well as the controllers of the system which include the evolutionary dynamics of the population games to control the input flow of each tank. Furthermore, we provide another subsystem to keep the total input flow under control. In this section, we show the system in standard notations along with how the tank system is connected to the controllers.

- Chapter 5: Stability Analysis.

In this chapter, we use the stability analysis material introduced in Chapter 3 to characterize the behavior of the system under study. Also a potential prob- 
lem that can cause some undesirable behavior for the system is investigated and a solution to this problem is suggested.

- Chapter 6: Disturbance Rejection.

This chapter of the thesis, adds another subsystem to the controllers of the system that improves the reliability and robustness when the system is influenced by an external disturbance. This leads to an improvement in the behavior of the system in Nash equilibrium seeking.

- Chapter 7: Global Convergence; Generalization.

Following the stability analysis in the foregoing chapters, an extension of a class of games that includes the WDS system and, in general, resource allocation problems is provided. This chapter shows that resource allocation problems that could be set out in this specified model represent stable behavior. Presenting the dynamical model of the generalized class of games and stability analysis are the objectives of this chapter.

3. Part III; Simulation and Conclusion.

The last two chapters of this thesis contain the simulation results of the system with proposed controllers and a conclusion that sums up the ideas and results of this work.

\subsection{Statement of Contributions}

The following is a list of contributions made in this thesis.

1. Chapter 4 suggests a solution approach for solving the problem of water distribution to $N$ tanks and Chapter 5 proves the stability and convergence of the system to the desired equilibrium points:

- Local convergence of the $N$-tank system to the desirable Nash equilibrium in 
connection with the Replicator Dynamic, Theorem 5.1.

- Improvement in behavior of the system by employing different classes of evolutionary dynamics such as the BNN and Smith dynamics, Theorem 5.2 and Theorem 5.3 .

2. Chapter 6 provides an extra subsystem to reject a certain type of disturbances:

- Disturbance rejection and zero error in converging to desired Nash equilibrium, Theorem 6.1.

3. Chapter 7 presents global convergence and stability as an improvement in system responses:

- Global asymptotic stability of the tank system, Theorem 7.1 , Theorem 7.2 and Theorem 7.3 .

- A generalization for a class of resource allocation problems and asymptotic stability of the suggested system, Theorem 7.4 . 


\section{Part I}

\section{Preliminaries/Background}




\section{Chapter 2}

\section{Population Games and Evolutionary Dynamics}

This chapter reviews preliminary background material in game theory. In Section 2.1 . population games are introduced and some basic definitions such as players, payoffs and equilibrium points of a population game, mainly taken from [36], are discussed. In order to analyze the behavior of a population game, evolutionary dynamics as a model of population games [36] as well as different classes of evolutionary dynamics are presented in Section 2.2 and some of the implications, found in [35, 36, 40], are reviewed.

\subsection{Population Games}

\subsubsection{Populations, Strategies and States}

Consider a large population of decision-makers known as players/agents that are playing a game by selecting among several available choices called strategies. In each game, there is a payoff associated with every strategy that could be gained by the agents who are playing the corresponding strategy.

To formally define a population game, assume there is a very large number of $m$ 
players $(m \rightarrow \infty)$ that have to play a set of available strategies denoted $S=\{1, \ldots, N\}$. During the game play, each player selects a strategy from the set $S$, so if $m_{i}$ players select the strategy $i$, we can show the fraction of $i$-strategists by $p_{i}=m_{i} / m$. Therefore, we can define a positively invariant set known as the simplex of the population as $\Delta=$ $\left\{p=\left[p_{1}, \ldots, p_{i}, \ldots, p_{N}\right]^{T} \in \mathbb{R}_{+}^{N}: \sum_{i \in S} p_{i}=1\right\}$ where $p$ as a vector of fractions of the players for each strategy is called the population state. The population state $p \in \Delta$ is also referred as strategy distribution over the set of strategies.

In the next part, more fundamental definitions of games are presented.

\subsubsection{Payoffs, Best Responses, Nash Equilibria}

We generally identify a game with its payoff function. A payoff function $f: \Delta \longrightarrow \mathbb{R}^{N}$ is a continuous mapping that assigns the population state a vector of payoffs; one payoff for each strategy.

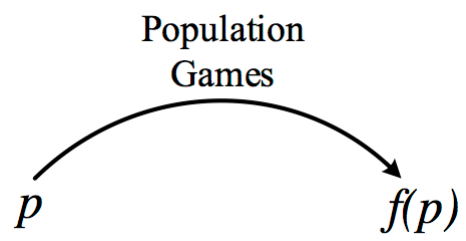

Figure 2.1: Population games as mappings from population state to payoffs.

We denote the vector of payoffs by $f(p)=\left[f_{1}(p), \ldots, f_{N}(p)\right]^{T}$ where $f_{i}(p)$ is a continuous payoff function of playing $i$-th strategy. In addition to continuity of $f$, we impose stronger assumptions such as Lipschitz continuity or continuous differentiability. We will use these properties later in the next chapters of this thesis. We can also define the average payoff of a population by

$$
\bar{f}=\sum_{i \in S} p_{i} f_{i}
$$

To describe the optimality in behavior of the players in a population, we define popula- 
tion's best response $b: \Delta \Longrightarrow S$, which specifies the strategies in $S$ that give the highest payoff to the agents who are playing those strategies

$$
b(p)=\underset{j \in S}{\operatorname{argmax}} f_{j}(p) .
$$

Also considering the simplex of the game, the mixed best response $B: \Delta \Longrightarrow \Delta$ is given by

$$
B(p)=\left\{y \in \Delta: y_{j}>0 \Rightarrow j \in b(p)\right\}
$$

Geometrically, $B(p)$ is the convex hull of the vertices of $\Delta$ corresponding to the elements of $b(p)$. A population state $p \in \Delta$ is a Nash Equilibrium $(N E)$ of the population game $f$ if each agent in the population selects a best response to $p$, i.e.,

$$
N E(f)=\{p \in \Delta: p \in B(p)\}
$$

similarly, if $p \in N E(f)$, every utilized strategy earns the maximum payoff

$$
\begin{aligned}
N E(f) & =\left\{p \in \Delta:\left[p_{i}>0 \Rightarrow f_{i}(p) \geqslant f_{j}(p)\right] \forall i, j \in S\right\} \\
& =\left\{p \in \Delta:\left[p_{i}>0 \Rightarrow f_{i}(p)=\max _{j \in S} f_{j}(p)\right] \forall i, j \in S\right\} .
\end{aligned}
$$

Since every strategy receives the maximum possible payoff for $p \in N E(f)$, if we denote the maximum payoff by $f^{*}$, we can write

$$
f_{i}(p)=f^{*}=\max _{j \in S} f_{j}(p) \quad \forall i \in b(p) \quad \text { and } \quad \forall j \in S
$$

However, in practice, computing the Nash equilibrium points of an ongoing population game is not as easy as the definitions are. In a population game with a large number of agents, it is usual to assume that players gradually revise their strategies to adjust their choices to the current strategic environment, i.e., they keep revising their strategies 
and interacting with other players so by repetitive interactions, they can reach a point where all of them are content. Although, by changing the selections of strategies, the population state varies in time, therefore, the payoff function $f(p)$, which is dependent on $p$, will change. The situation can even get more difficult if the payoff functions are complicated functions highly dependent on $p$, so the natural question is whether or not players' revisions lead to the Nash Equilibria of the game. Therefore, a powerful method that can analyze the behavior of the population and study the evolution of the players' strategies is needed. Evolutionary dynamics, which is described in detail in the next section, is the most comprehensive tool to achieve this goal.

\subsection{Evolutionary Dynamics}

A traditional approach to prediction in games, equilibrium analysis, is based on assumptions about what players know about the game. The assumptions such as full rationality of players - they completely understand the game they are playing and anticipate how others will act - are strong and demanding, particularly in applications in which the number of agents is large.

An alternate method, which is appropriate for repetitive interactions among a large number of players, proceeds through a dynamic, disequilibrium analysis. From this point of view, agents attempt to adjust and revise their strategy selections with respect to their current strategies and the population state as soon as they receive a revision opportunity. To model a game in this setup, another tool, called a revision protocol, is needed to specify the procedure of how and when agents reselect their strategies. This protocol shows what information is available to the agents and how it is used to change the strategies in order to achieve the goal of game playing which is getting a better payoff.

A population game, a population size and a revision protocol together, produce a prediction process on the set of population state, which is distribution over pure strategies, 
that can be approximated by a deterministic trajectory. This trajectory is obtained as a solution of an ordinary differential equation called the mean dynamic that is induced by the game and the protocol.

From a slightly different point of notion, we can view a revision protocol as a map from population games to differential equations and a prediction to the population states as the output of the differential equations. This map is called the evolutionary dynamic that reflects the characteristics of the revision protocol and the population game that generate them.

In the next section, a formal definition of revision protocols and a general model of evolutionary dynamic, the mean dynamic, are presented.

\subsubsection{Revision Protocols, Mean Dynamics}

To specify the process of playing a game, we consider how agents make their decisions. Revision protocols describe how and when agents switch strategies and what information they use in order to revise their selections. In a population game, once in a while, agents are given opportunities to change their choices. Given a payoff vector and population state of a population game, each agent employs a revision protocol to switch to a better strategy. Considering $f: \Delta \longrightarrow \mathbb{R}^{N}$ as a payoff function of a population game (identification of game) and $S$ as an action set, a formal definition of revision protocol, denoted by $\rho$, is presented in the following definition.

Definition 2.1 ([36], p.121). A revision protocol $\rho$ is a map $\rho(f, p): \mathbb{R}^{N} \times \Delta \longrightarrow \mathbb{R}_{+}^{N \times N}$. The scalar protocol $\rho_{i j}$ is called the conditional switch rate from strategy $i \in S$ to strategy $j \in S$ given the population state $p$ and the payoff vector $f$.

Having the definition of revision protocols in mind, the following describes the process of evolution in a population game with a large number of agents. Assume each agent is equipped with a stochastic alarm clock that is independent of others' clocks. Each 
time a clock rings, it shows that the corresponding player is given an opportunity to revise his choice. If an agent is playing strategy $i$, by the arrival of a switching signal, he considers switching to another strategy $j \neq i$ using a conditional switch rate $\rho_{i j}$. Therefore, recalling $m$ as the total number of agents and $m_{i}$ as the number of agents playing $i$-th strategy, the expected number of $i$-strategists that lead to switch to strategy $j$ is $m_{i} \rho_{i j}$ or in terms of the strategy distribution it will be $\frac{m_{i}}{m} \rho_{i j}=p_{i} \rho_{i j}$ which we call outflow from $i$-th strategy. Similarly, the total outflow of $i$-strategists that tend to switch to all other strategies is given by $p_{i} \sum_{j \in S} \rho_{i j}$. Likewise, we can consider the same procedure of strategy selection for the agents who want to migrate from other strategies to strategy $i$. So the total inflow to strategy $i$ will be $\sum_{j \in S} p_{j} \rho_{j i}$. Thus, the expected change in the proportion of agents using $i$-th strategy, denoted $d p_{i}$, in $d t$ time is defined as a difference between the inflow and the outflow as follows

$$
d p_{i}=\left(\sum_{j \in S} p_{j} \rho_{j i}-p_{i} \sum_{j \in S} \rho_{i j}\right) d t
$$

Hence, the mean dynamic induced by population game $f$, describing the growth rate with respect to time of $i$-th strategy, is

$$
\dot{p}_{i}=\sum_{j \in S} p_{j} \rho_{j i}-p_{i} \sum_{j \in S} \rho_{i j} .
$$

The definition below formally defines mean dynamic and evolutionary dynamic.

Definition 2.2 ([36], p.124). The mean dynamic of population $f$ is a set of ordinary differential equations, describing the evolution in time (growth rate) of the population state $p$ under a given revision protocol $\rho$, that is shown by

$$
\dot{p}=V(f, p), \quad \text { where } \quad V_{i}(f, p)=\sum_{j \in S} p_{j} \rho_{j i}(f, p)-p_{i} \sum_{j \in S} \rho_{i j}(f, p)
$$

and, in general, is called the evolutionary dynamic. 
Notice that on condition that $V(f, p): \mathbb{R}^{N} \times \Delta \longrightarrow \mathbb{R}^{N}$ is Lipschitz continuous 1 , existence and uniqueness of solution to equation (2.8) follow from standard results found in [25, 36] . Therefore, we ensure the Lipschitz continuity of $V(f, p)$ by assuming that $f$ and $\rho(f, p)$ are Lipschitz continuous. That being the case, by considering the Definitions 2.1 and 2.2 as well as the description provided in Section 2.2, one can conclude that evolutionary dynamic is a mapping from payoff function $f$ to population state of the game under which the mean dynamics are generated.

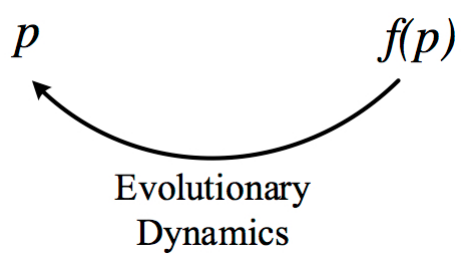

Figure 2.2: Evolutionary dynamics as mappings from payoffs to population state.

Regarding Nash equilibrium points of a population game reviewed in Section 2.1.1, one might be interested in investigating the connection of $N E$ 's of a game and the corresponding mean dynamic that is induced by the game. Evolutionary dynamic is called to be at rest point or to be converged to its equilibrium points if $V(f, p)=0$, therefore, the task to find a link between equilibrium points of evolutionary dynamic and $N E$ 's of the underlying game reduces to the problem of relating equations 2.4 and 2.5 to equation (2.8) when the right hand side of the latter is zero.

In the next section, two main properties regarding the evolutionary dynamic are discussed. These fundamental properties are utilized to make a connection between equilibrium points of evolutionary dynamic and the Nash equilibria of the corresponding population game.

\footnotetext{
${ }^{1}$ The function $V: D \longrightarrow \mathbb{R}^{N},\left(D \subseteq \mathbb{R}^{N}\right)$, is Lipschitz continuous if there exists a constant $K>0$ such that $\|V(y)-V(x)\|_{2} \leqslant K\|y-x\|_{2}$, where $\|\cdot\|_{p}$ denotes $p$-norm defined as $\|x\|_{p}=\left(\left|x_{1}\right|^{p}+\cdots+\left|x_{N}\right|^{p}\right)^{1 / p}$.
} 


\subsubsection{Positive Correlation, Nash Stationary}

Evolutionary dynamic introduced in the previous section provides a way of analyzing the game under which the mean dynamic is produced. However, it is required to determine whether a revision protocol that leads to the corresponding evolutionary dynamic of the underlying game is appropriate for using in a given application or not. A part of this investigation is to evaluate how $N E$ 's of a game are connected to equilibrium points of its mean dynamic. For this reason, the following two properties of evolutionary dynamics, introduced in [20, 35, 36], are needed:

- Nash Stationary (NS): $V(f, p)=0$ if and only if $p \in N E(f)$, and

- Positive Correlation (PC): $V(f, p) \neq 0$ implies that $V(f, p)^{T} f(p)>0$.

Nash stationary requires that rest points of evolutionary dynamics coincide with $N E$ 's of the corresponding population game, but positive correlation asks that whenever evolutionary dynamic is not at rest, population growth rates of the corresponding game are positively correlated with its payoffs.

Nash stationary property can be broken down into two distinct conditions. First, (NS) asks that Nash equilibrium of the game $f$ be the rest point of its evolutionary dynamics $V(f, p)$. If a population state $p$ is a $N E$, no agent benefits from switching strategies, therefore, there is no change in population state, i.e., $\dot{p}=V(f, p)=0$. Second, (NS) requires that every rest point of $V(f, p)$ be a Nash equilibrium of $f$. If population state $p$ is not a $N E$, then there are agents who can gain better payoffs by revising their choices, therefore, they will switch, which means that the game is not at rest. Thus, one can consider (NS) as a restriction on rest points and Nash equilibria of evolutionary dynamics and population games, respectively.

On the other hand, positive correlation can be assumed as a constraint on direction of motion in the process of a game play. The intuition behind $(\mathrm{PC})$ is that when a payoff decreases, the corresponding population growth rate decreases as well and whenever a 
payoff is higher, more players tend to play that strategy, so the corresponding population growth rate increases. Thus, if agents keep playing the game with this logic, only the strategies with the highest payoffs will survive which means that the game will move toward converging to $N E$ 's.

In the next section, different classes of evolutionary dynamic with specified prototypes for the revision protocols along with the discussion about the (PC) and (NS) properties are presented.

\subsubsection{Classes of Evolutionary Dynamics}

In this section, we present different types of revision protocol that lead to different evolutionary dynamics. We introduce some conditions on protocols to get different types of dynamics such as Imitative Dynamics, Excess Payoff Target and Pairwise Comparison Dynamics.

\section{Pairwise Proportional Imitative Protocols and Dynamics}

The revision protocol $\rho$ is pairwise proportional if the conditional switch rate $\rho_{i j}$ under which an agent switches from his current strategy $i$ to strategy $j$, depends on the popularity of $j$-th strategy and the difference between the payoffs of the corresponding strategies. In this type of protocol, switching agents compare their current strategies' payoffs with the payoffs of the strategies they are looking forward to switching to as well as considering their popularity among other agents in the population. So this protocol can be modeled as the following:

$$
\rho_{i j}=p_{j}\left[f_{j}(p)-f_{i}(p)\right]_{+},
$$


where the function $[x]_{+}: \mathbb{R} \longrightarrow \mathbb{R}_{+}$, defined as

$$
[x]_{+}=\left\{\begin{array}{ll}
x & x>0 \\
0 & x \leqslant 0
\end{array},\right.
$$

is a projection that ignores the negative parts of $x$. This type of projection helps agents determine when is the best time to switch to better strategies by comparing payoffs. As mentioned before, the number (or proportion) of the agents who are playing the strategy that is going to be chosen, is important because the switching agents also consider the popularity of that strategy which means that unused strategies $\left(p_{i}=0\right)$, regardless of having higher payoffs, remain untouched.

This type of revision protocol leads to the most studied evolutionary dynamic called the Replicator Dynamic. By substituting equation (2.9) in 2.8), we obtain

$$
\begin{aligned}
\dot{p}_{i} & =\sum_{j \in S} p_{j} p_{i}\left[f_{i}(p)-f_{j}(p)\right]_{+}-p_{i} \sum_{j \in S} p_{j}\left[f_{j}(p)-f_{i}(p)\right]_{+} \\
& =p_{i} \sum_{j \in S} p_{j}\left(\left[f_{i}(p)-f_{j}(p)\right]_{+}-\left[f_{j}(p)-f_{i}(p)\right]_{+}\right) .
\end{aligned}
$$

Due to the fact that $x=[x]_{+}-[-x]_{+}$, we arrive at

$$
\begin{aligned}
\dot{p}_{i} & =p_{i} \sum_{j \in S} p_{j}\left(f_{i}(p)-f_{j}(p)\right)=p_{i}\left(f_{i}(p) \sum_{j \in S} p_{j}-\sum_{j \in S} p_{j} f_{j}(p)\right) \\
& =p_{i}\left(f_{i}(p)-\bar{f}(f, p)\right)=p_{i} \hat{f}_{i}(f, p),
\end{aligned}
$$

where

$$
\bar{f}(f, p)=\sum_{j \in S} p_{j} f_{j}(p) \quad \text { and } \quad \hat{f}_{i}(f, p)=f_{i}(p)-\bar{f}(f, p),
$$

are the average payoff and the excess payoff functions, respectively. Under this dynamic, the percentage growth rate of each strategy $\dot{p}_{i} / p_{i}$ is equal to that strategy's excess payoff. In order to analyze behavior of the Replicator Dynamic in the process of a game play, 
we consider the theorem below that captures the positive correlation property of the corresponding evolutionary dynamics.

Theorem 2.1 ([36], Theorem 5.4.9, p.163). The Replicator Dynamic satisfies (PC).

Proof: To show (PC), we need $V(f, p)^{T} f(p)>0$ for any population state at which $V(f, p) \neq 0$. Let $p \in \Delta$ be the state of a population. It is clear that $\sum_{i \in S} p_{i}=1$, so by taking the derivative with respect to time, we get

$$
\sum_{i \in S} \dot{p}_{i}=\sum_{i \in S} V_{i}(f, p)=0
$$

We define

$$
\begin{array}{lll}
S_{+} & =\left\{i \in S: p_{i}>0 \quad \text { and } \quad\right. & \left.V_{i}(f, p)>0\right\} \quad \text { and } \\
S_{-}=\left\{i \in S: p_{i}>0 \quad\right. \text { and } & \left.V_{i}(f, p)<0\right\}
\end{array}
$$

where $S_{+}$and $S_{-}$are the strategy sets in which the growth rate of the population state is strictly positive and negative, respectively. From equation (2.13), it is clear that

$$
\sum_{i \in S} V_{i}(f, p)=\sum_{i \in S_{+}} V_{i}(f, p)+\sum_{i \in S_{-}} V_{i}(f, p)=0 .
$$

Now starting with the inner product of the population growth rate and the payoff vector, we arrive at

$$
\begin{aligned}
V \cdot f=V(f, p)^{T} f(p) & =\sum_{i \in S_{+}} V_{i} f_{i}+\sum_{i \in S_{-}} V_{i} f_{i} \\
& \geqslant \min _{i \in S_{+}} f_{i} \sum_{i \in S_{+}} V_{i}+\max _{i \in S_{-}} f_{i} \sum_{i \in S_{-}} V_{i},
\end{aligned}
$$

where we can justify the RHS of the above inequality by equations (2.11) and (2.14); it is easy to conclude that $f_{i}(p)>\bar{f}(p)$ for any $i \in S_{+}$, and also $f_{i}(p)<\bar{f}(p)$ for all $i \in S_{-}$. So, $\min _{i \in S_{+}} f_{i}(p)>\max _{i \in S_{-}} f_{i}(p)$. Thus, by equation 2.15,

$$
V(f, p)^{T} f(p) \geqslant\left(\min _{i \in S_{+}} f_{i}-\max _{i \in S_{-}} f_{i}\right) \sum_{i \in S_{+}} V_{i}>0
$$


which proves that the Replicator Dynamic satisfies (PC).

However, to analyze the property of (NS) for the Replicator Dynamic, it is easy to confirm that equation (2.11) admits two different sets of rest points. In the first case, $f_{i}(p)=\bar{f}(p)=f^{*}$ for $p_{i}>0$ only happens when, by equations (2.5) and (2.6), $p$ is a Nash equilibrium. But the second scenario occurs when $p_{i}=0$ (on the boundary of the simplex) that, by definition, is not a $N E$ of the game. So clearly, the Replicator Dynamic can admit non-Nash Equilibria. Therefore, we deduce that the Nash Stationary property is not satisfied by the Replicator Dynamic.

Next we introduce another set of evolutionary dynamics that shows more desirable behavior.

\section{Excess Payoff Target Protocols and Dynamics}

In Excess Payoff Target dynamic (EPT), agents are looking for strategies with higher payoffs in comparison with the average payoff of the population. When a player receives an opportunity to revise his choice, he randomly selects a strategy (called the target strategy) and compares its payoff to the average payoff. In this type of revising procedure, the current payoff of players' strategies and popularity of the target choice are not considered. Therefore, unused strategies could be chosen and played by the agents. If the selected strategy has a higher payoff, agents will switch to and will play it until they receive another chance of strategy revision. Since players' current payoffs are not important, we can rewrite the conditional switch rates as follows:

$$
\rho_{i j}(f, p)=\tau_{j}(f, p)
$$

where $\tau_{j}: \mathbb{R}^{N} \times \Delta \longrightarrow \mathbb{R}_{+}$shows that the protocol is only dependent on the target strategy $j$. To show the mean dynamic generated by this type of protocol, we substitute equation 2.18 in the equation of the mean dynamic 2.8. So we obtain the EPT 
dynamic as follows:

$$
\dot{p}_{i}=\tau_{i}(f, p)-p_{i} \sum_{j \in S} \tau_{j}(f, p)
$$

A prototype of the EPT dynamic is the Brown-Von Neumann-Nash dynamic (BNN) with the revision protocol

$$
\tau_{j}(f, p)=\left[\hat{f}_{j}(f, p)\right]_{+}
$$

where $\hat{f}_{j}$ is defined in equation 2.12 . Considering this protocol, by substituting in equation (2.8) the BNN dynamic will take the form of

$$
\dot{p}_{i}=\left[\hat{f}_{i}(f, p)\right]_{+}-p_{i} \sum_{j \in S}\left[\hat{f}_{j}(f, p)\right]_{+}
$$

The theorem below discusses the positive correlation and Nash stationary property for the BNN dynamic.

Theorem 2.2 ([36], Theorem 5.5.2, p.167). The BNN dynamic satisfies (PC) and (NS).

Proof: Consider the BNN dynamic, presented in equation 2.21). To show the (PC) property for this dynamic, we have to prove that $\dot{p} \neq 0$ implies $f^{T} \dot{p}>0$. So we start with the inner product as follows:

$$
\begin{aligned}
V(f, p)^{T} f(p) & =\sum_{i \in S} V_{i} f_{i}=\sum_{i \in S} f_{i}\left(\left[\hat{f}_{i}\right]_{+}-p_{i} \sum_{j \in S}\left[\hat{f}_{j}\right]_{+}\right) \\
& =\sum_{i \in S} f_{i}\left[\hat{f}_{i}\right]_{+}-\sum_{i \in S} f_{i} p_{i} \sum_{j \in S}\left[\hat{f}_{j}\right]_{+} \\
& =\sum_{i \in S}\left[\hat{f}_{i}\right]_{+}\left(f_{i}-\sum_{j \in S} p_{j} f_{j}\right)=\sum_{i \in S} \hat{f}_{i}\left[\hat{f}_{i}\right]_{+},
\end{aligned}
$$

where the last equality is due to equation 2.12 . Since it is assumed that $\dot{p} \neq 0$, there are agents who are currently switching to other strategies to get better payoffs. Due to the definition of the protocol that allows an agent to switch if and only if he receives a payoff higher than the average, there exists at least a strategy that gains a higher payoff 
than the average payoff, i.e., there exists a $j$ such that $\hat{f}_{j}>0$. Therefore, the equation above is always positive definite whenever $\dot{p} \neq 0$.

For the (NS) property, we need to prove that $V(f, p)=0$ if and only if $p \in N E(f)$. $(\Leftarrow)$ :

Let $p \in N E(f)$ be a population state for game $f$. By the definition of Nash equilibrium in equations (2.5) and (2.6), we have

$$
f_{i}=f^{*}=\max _{j \in S} f_{j}(p), \quad \forall i \in b(p) \quad \text { and } \quad p_{i}>0
$$

and also

$$
\bar{f}=\sum_{i \in S} p_{i} f_{i}=f^{*} \sum_{i \in S} p_{i}=f^{*} \quad \text { and } \quad \hat{f}_{i}=f_{i}-\bar{f}=0, \quad \forall p \in N E
$$

Therefore, by substituting the above equation in equation 2.21 , we obtain $\dot{p}=0$. $(\Rightarrow)$ :

We need to show that $\dot{p}=0$ implies $p \in N E(f)$. Suppose $p \notin N E(f)$ which means that for $p_{i}>0$ there exists a $j \in S$ such that $f_{i}<f_{j}$. Therefore, $i$-strategists find $j$-th strategy more attractive than their own, so they will change their strategies whenever they receive a revision opportunity. This means that $\dot{p}_{i} \neq 0$ and accordingly $\dot{p} \neq 0$, which is a contradiction. Therefore, $p \in N E(f)$ if and only if $\dot{p}=0$, as required.

Considering the Replicator Dynamic in comparison with the BNN dynamic, one will notice that the problem of admitting non-Nash equilibrium points can be solved by utilizing the EPT revision protocol instead of pairwise proportional imitative protocols. Moreover, good unused strategies with higher payoffs, which would remain untouched in the Replicator Dynamic, could be involved in the game-play by choosing the BNN dynamic to characterize a population behavior. However, in analyzing the behavior of a population game, the amount of data required by either the Replicator Dynamic or the BNN dynamic is high. To clarify, consider the dynamics shown in equations 2.11) 
and (2.21). At each time agents change their strategies, they need information about the payoffs of all of the strategies in order to calculate the average payoff of the population. Additionally, in the Replicator Dynamic not only agents ask for the population payoff, but they also need the population state. So playing large population games with these high information demanding protocols is difficult. For this reason, another evolutionary dynamic that facilitates the game-play and addresses these problems is introduced in the next section.

\section{Pairwise Comparison Protocols and Dynamics}

In this type of revision protocol, whenever agents are given opportunities to revise their strategies, they only consider the payoff of the selected strategy. At each revision time, players compare their own payoffs with the payoff of the strategy they are switching to. So the revision protocol of this class of evolutionary dynamic can be written as

$$
\rho_{i j}(f, p)=\rho_{i j}\left(f_{j}-f_{i}\right)
$$

which means that only the payoff of current and target strategies are important to the agents. The Smith dynamic with a revision protocol given by

$$
\rho_{i j}\left(f_{j}-f_{i}\right)=\left[f_{j}(p)-f_{i}(p)\right]_{+},
$$

is a prototype of this class of evolutionary dynamic. As can be seen, neither the average payoff nor the population state is needed in this protocol. By substituting this protocol in equation (2.8), we get the Smith dynamic as follows:

$$
\dot{p}_{i}=\sum_{j \in S} p_{j}\left[f_{i}(p)-f_{j}(p)\right]_{+}-p_{i} \sum_{j \in S}\left[f_{j}(p)-f_{i}(p)\right]_{+} \cdot
$$

The theorem below introduces the (PC) and (NS) properties for the Smith dynamic. 
Theorem 2.3 ([36], Theorem 5.6.2, p.170). The Smith dynamic satisfies (PC) and (NS).

Proof: By starting from the inner product, we get

$$
\begin{aligned}
V(f, p)^{T} f(p) & =\sum_{i \in S} f_{i}\left(\sum_{j \in S} p_{j}\left[f_{i}(p)-f_{j}(p)\right]_{+}-p_{i} \sum_{j \in S}\left[f_{j}(p)-f_{i}(p)\right]_{+}\right) \\
& =\sum_{i \in S} \sum_{j \in S} f_{i} p_{j} \rho_{j i}-\sum_{i \in S} \sum_{j \in S} f_{i} p_{i} \rho_{i j} .
\end{aligned}
$$

By switching $i$ and $j$ in the first term of the RHS, we get

$$
\begin{aligned}
V(f, p)^{T} f(p) & =\sum_{j \in S} \sum_{i \in S} f_{j} p_{i} \rho_{i j}-\sum_{i \in S} \sum_{j \in S} f_{i} p_{i} \rho_{i j} \\
& =\sum_{i \in S} \sum_{j \in S} f_{j} p_{i} \rho_{i j}-\sum_{i \in S} \sum_{j \in S} f_{i} p_{i} \rho_{i j} \\
& =\sum_{i \in S} \sum_{j \in S} p_{i}\left(f_{j}-f_{i}\right) \rho_{i j}=\sum_{i \in S} \sum_{j \in S} p_{j}\left(f_{j}-f_{i}\right)\left[f_{j}-f_{i}\right]_{+}
\end{aligned}
$$

where the last equality is due to equation 2.24). Similar to Theorem 2.2, assume that $\dot{p}_{i} \neq 0$, so there are agents who are currently switching to and from strategy $i$. Due to the definition of the protocol that allows an agent to switch if and only if he receives a payoff higher than his own, there exists at least a $j \in S$ such that $f_{j}>f_{i}$. Therefore, the equation above is always positive definite whenever $\dot{p} \neq 0$, as required.

For (NS), we need to show that $\dot{p}=0$ if and only if $p \in N E(f)$. $(\Leftarrow)$ :

Let $p \in N E(f)$ be a population state for the game $f$. By the definition of Nash equilibrium in equations (2.5) and (2.6), we have

$$
f_{i}=f_{j}=f^{*}=\max _{k \in S} f_{k}(p), \quad \forall i, j \in b(p) \quad \text { and } \quad p_{i}, p_{j}>0 .
$$

Therefore, by substituting the above equation in equation 2.25 , we obtain $\dot{p}=0$. $(\Rightarrow)$ : 
We need to show that $\dot{p}=0$ implies $p \in N E(f)$. Suppose $p \notin N E(f)$ which means that for $p_{i}>0$ there exists a $j \in S$ such that $f_{i}<f_{j}$. Therefore, $i$-strategists find $j$-th strategy more attractive than their own, so they will change their strategies whenever they receive revision opportunities. This means that $\dot{p}_{i} \neq 0$ and accordingly $\dot{p} \neq 0$, which is a contradiction. Therefore, $p \in N E(f)$ if and only if $\dot{p}=0$.

Table 2.1 below summarizes the three models of population game with different revision protocols, described in this section, with respect to different properties (PC), (NS) and the information needed:

\begin{tabular}{|c|c|c|c|c|}
\hline $\begin{array}{c}\text { Evolutionary } \\
\text { Dynamics }\end{array}$ & Prototype & $\mathrm{PC}$ & NS & Less Data \\
\hline $\begin{array}{c}\text { Pairwise Proportional } \\
\text { Imitation }\end{array}$ & $\begin{array}{l}\text { Replicator } \\
\text { Dynamic }\end{array}$ & Yes & No & No \\
\hline Excess Payoff Target & $\begin{array}{c}\text { BNN } \\
\text { Dynamic }\end{array}$ & Yes & Yes & No \\
\hline Pairwise Comparison & $\begin{array}{c}\text { Smith } \\
\text { Dynamic }\end{array}$ & Yes & Yes & Yes \\
\hline
\end{tabular}

Table 2.1: Classes of evolutionary dynamics and their properties.

Next, we present how evolutionary dynamics and population games are connected.

\subsubsection{Connection of Games and Evolutionary Dynamics}

In this section, we discuss how agents gain payoffs by playing a game and guess their future strategy selection by analyzing the game behavior via evolutionary dynamic, i.e., we describe how population games and evolutionary dynamics are connected to make the game playable in a desirable way for agents.

As reviewed in the last section, we know that population games are mappings from population state to payoffs. This means that players are able to calculate their payoffs by selecting their choices and playing them through the population game. On the other hand, recalling evolutionary dynamic as a mapping from payoffs to population state, 
agents with their payoffs in hand can utilize a revision protocol to build a dynamical model (mean dynamic) of the game they are playing, where the solution of this model avails themselves to observe and analyze the process of game evolution with respect to the protocol in use.

Considering these mappings, shown in Figures 2.1 and 2.2, we can view the connection of evolutionary dynamic with the corresponding population game, under which the mean dynamic is derived, as a closed-loop system. The following figure shows this interconnection:

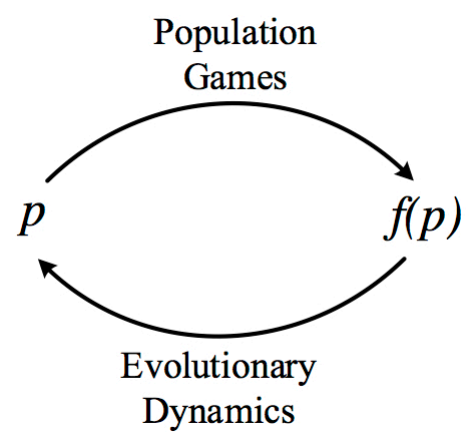

Figure 2.3: Connection of population games and evolutionary dynamics.

This closed loop inspired us to model this interconnection as a negative feedback interconnection of two input-output systems as shown below

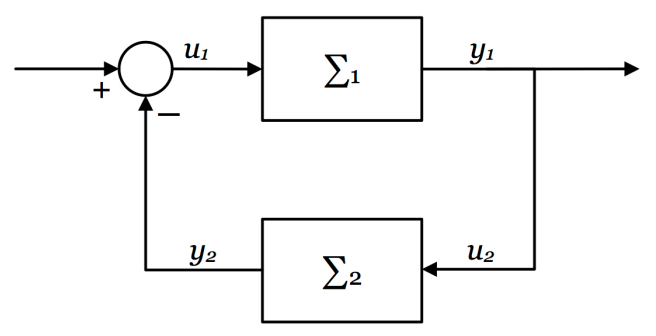

Figure 2.4: Negative feedback interconnection of two systems.

where $\Sigma_{1}$ and $\Sigma_{2}$ are input-output models of population games and evolutionary dynamics, respectively.

In [18], the authors showed that for a class of population games called the stable games [20, 35, 36] that satisfy the following inequality:

$$
(a-b)^{T}(f(a)-f(b)) \leqslant 0
$$


where $a$ and $b$ are two different population states defined as $a=\left[a_{1}, \ldots, a_{i}, \ldots, a_{N}\right]^{T}$ and $b=\left[b_{1}, \ldots, b_{i}, \ldots, b_{N}\right]^{T}$ for all $i$ in action set of the game, the negative feedback interconnection with certain classes of evolutionary dynamic will converge to the set of $N E$ 's of the corresponding stable game. But to analyze this feedback interconnection system, we need stability analysis tools such as Lyapunov stability and passivity theorems. In the next chapter, basic tools needed for characterizing the convergence of the systems to $N E$ 's are provided. 


\section{Chapter 3}

\section{Stability and Passivity}

In this chapter, we provide background about stability analysis of dynamical systems. In Section 3.1, definitions of passivity, Lyapunov stability theorem and some basic lemmas are presented. Section 3.2 contains stability analysis of negative feedback interconnections of input-output dynamical systems. In Section 3.3, passivity of systems described by transfer functions are discussed. The definitions and theorems that will be used in this chapter and the next parts of this thesis are adapted from [25] and slightly modified where needed.

\subsection{Passivity and Lyapunov Stability}

Consider a dynamical system represented by

$$
\begin{aligned}
& \dot{x}=f(x, u), \\
& y=h(x, u),
\end{aligned}
$$

where $x \in \mathcal{X}$ is the state, $u \in \mathcal{U}$ is the input, $f: \mathcal{X} \times \mathcal{U} \longrightarrow \mathbb{R}^{n}$ is a locally Lipschitz vector field and $h: \mathcal{X} \times \mathcal{U} \longrightarrow \mathbb{R}^{p}$ is continuous with $f(0,0)=0$ and $h(0,0)=0$. We let $\mathcal{X} \subset \mathbb{R}^{n}$ and $\mathcal{U} \subset \mathbb{R}^{p}$ be the state space and the class of piecewise continuous input 
signal, respectively. The system has the same number of inputs $u$ and outputs $y$. The definition below introduces passive systems:

Definition 3.1 ([25], Definition 6.3, p.236). Consider the system of form (3.1). If there exists a continuously differentiable nonnegative function $L: \mathcal{X} \longrightarrow \mathbb{R}$ called the storage function such that $\forall(x, u) \in \mathcal{X} \times \mathcal{U}$

- $u^{T} y=\dot{L}=\frac{\partial L}{\partial x} f(x, u)$, then the system is lossless.

- $u^{T} y \geqslant \dot{L}$, the system is called passive.

- $u^{T} y \geqslant \dot{L}+\varphi(x)$ where $\varphi(x)>0 \quad \forall x \neq 0$, the system is strictly passive.

- $u^{T} y \geqslant \dot{L}+y^{T} \varphi_{1}(y), y^{T} \varphi_{1}(y)>0 \quad \forall y \neq 0$, the system is output strictly passive.

- $u^{T} y \geqslant \dot{L}+u^{T} \varphi_{2}(u), u^{T} \varphi_{2}(u)>0 \quad \forall u \neq 0$, the system is called input strictly passive.

By keeping the definition of passivity in mind, we present the following theorem and lemma to characterize the stability of dynamical systems in the form of (3.1).

Theorem 3.1 ([25], Theorem $4.1 \& 4.2$, p.114 \&124). Let $x=0$ be an equilibrium point for the system in the form of (3.1) and $\mathcal{X} \subset \mathbb{R}^{n}$ be a domain containing $x=0$. Let $\mathcal{V}: \mathcal{X} \longrightarrow \mathbb{R}$ be a continuously differentiable positive definite candidate Lyapunov function such that

$$
\begin{gathered}
\mathcal{V}(0)=0 \quad \text { and } \quad \mathcal{V}(x)>0 \quad \forall x \in \mathcal{X}-\{0\} \\
\dot{\mathcal{V}}(x) \leqslant 0 \quad \forall x \in \mathcal{X}
\end{gathered}
$$

then, $x=0$ is stable. Moreover, if

$$
\dot{\mathcal{V}}(x)<0 \quad \forall x \in \mathcal{X}-\{0\}
$$


then $x=0$ is asymptotically stable. Furthermore, if $\mathcal{X}=\mathbb{R}^{n}$ and

$$
\begin{aligned}
\|x\| & \rightarrow \infty \Longrightarrow \mathcal{V}(x) \rightarrow \infty, \quad \text { (Radially Unbounded) } \\
\dot{\mathcal{V}}(x)<0 \quad \forall x \neq 0 &
\end{aligned}
$$

then $x=0$ is globally asymptotically stable.

Proof: See [25], Theorem 4.1.

The lemma below relates Lyapunov stability to passive systems:

Lemma 3.1 (25], Lemma 6.7, p.243). Consider the system of form 3.1). The origin of $\dot{x}=f(x, 0)$ is asymptotically stable if the system is strictly passive with a positive definite storage function. Furthermore, if the storage function is radially unbounded, the origin will be globally asymptotically stable.

Proof: Suppose that the system in form (3.1) is strictly passive with a positive definite storage function $\mathcal{V}: \mathcal{X} \longrightarrow \mathbb{R}$. By Definition 3.1, we have

$$
\dot{\mathcal{V}}(x) \leqslant u^{T} y-\varphi(x)
$$

where $\varphi(x)$ is positive definite. If we let $u=0, \dot{\mathcal{V}}$ satisfies the inequality $\dot{\mathcal{V}} \leqslant-\varphi(x)<0$ $\forall x \in \mathcal{X}-\{0\}$. Therefore, by Theorem 3.1 , the origin is asymptotically stable and if the storage function is radially unbounded, $x=0$ is globally asymptotically stable.

Remark 3.1. In Lemma 3.1, the connection of passivity to Lyapunov stability has been captured. It is shown that, under some conditions, a storage function can be used as a Lyapunov function for the same system where the input is zero $u=0$. Due to the definition of passivity presented in Definition 3.1, positive semi-definiteness of the storage function is a requirement for the system under study. However, according to Theorem 3.1. Lyapunov functions are needed to be positive definite. Therefore, a stronger condition 


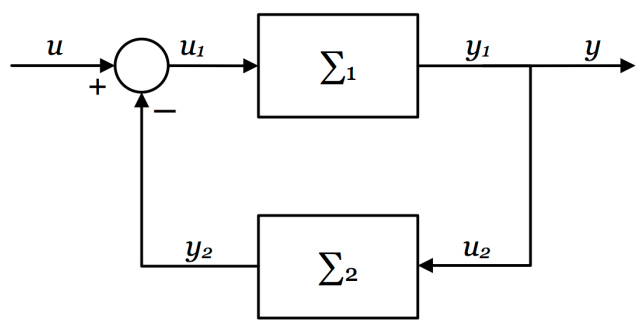

Figure 3.1: Negative feedback interconnection of the systems in the form of (3.1).

that is positive definiteness is assumed for the storage functions to be used as Lyapunov functions in Lemma 3.1 and later in this thesis.

In the next section, we connect input-output dynamical systems in the form of a negative feedback interconnection. A detailed discussion about the stability and passivity of the connection under some conditions is provided.

\subsection{Feedback Connection}

The two theorems below consider the passivity of the negative feedback interconnection of dynamical systems of form (3.1).

Theorem 3.2 ([25], Theorem 6.1, p.247). Consider the negative feedback interconnection of two time-invariant dynamical systems of form (3.1). The closed-loop system shown in Figure 3.1 is passive if $\Sigma_{1}$ and $\Sigma_{2}$ are passive with positive definite storage functions $L_{1}$ and $L_{2}$, respectively. Furthermore, if both of the systems are strictly passive, the negative feedback interconnection is strictly passive.

Moreover, if the interconnection is strictly passive, for $u=0$ the origin of the negative feedback interconnected system is asymptotically stable.

Proof: Consider the negative feedback interconnection between two systems $\Sigma_{1}$ and 
$\Sigma_{2}$ in the form of

$$
\Sigma_{1}:\left\{\begin{array}{l}
\dot{x_{1}}=f_{1}\left(x_{1}, u_{1}\right) \\
y_{1}=h_{1}\left(x_{1}, u_{1}\right)
\end{array} \quad, \quad \Sigma_{2}:\left\{\begin{array}{l}
\dot{x_{2}}=f_{2}\left(x_{2}, u_{2}\right) \\
y_{2}=h_{2}\left(x_{2}, u_{2}\right)
\end{array}\right.\right.
$$

where $u_{1}=u-y_{2}$ and $u_{2}=y_{1}$. Let $L_{1}\left(x_{1}\right)$ and $L_{2}\left(x_{2}\right)$ be the positive definite storage functions for $\Sigma_{1}$ and $\Sigma_{2}$, respectively. Consider the positive definite storage function $L(x)=L_{1}\left(x_{1}\right)+L_{2}\left(x_{2}\right)$ for the feedback interconnection system. From Figure 3.1 , by Definition 3.1, we have

$$
\dot{L}(x, u)=\dot{L_{1}}+\dot{L_{2}} \leqslant u_{1}^{T} y_{1}+u_{2}^{T} y_{2}=\left(u-y_{2}\right)^{T} y_{1}+y_{1}^{T} y_{2}=u^{T} y
$$

Therefore,

$$
\dot{L}(x) \leqslant u^{T} y
$$

and the negative feedback interconnected system is passive. Similarly, if both systems are strictly passive, we arrive at

$$
\dot{L}(x)=\dot{L}_{1}+\dot{L}_{2} \leqslant u^{T} y-\varphi\left(x_{1}, x_{2}\right)
$$

where $\varphi\left(x_{1}, x_{2}\right)=\varphi_{1}\left(x_{1}\right)+\varphi_{2}\left(x_{2}\right)$ and from Definition 3.1, it is positive definite. Thus, the feedback interconnection will be strictly passive.

Now if we let $u=0$, we get $\dot{L}(x)<0$, so by Lemma 3.1, the origin of the feedback interconnection of the two strictly passive systems is asymptotically stable.

In the next theorem, instead of connecting two systems, we connect three dynamical systems. The theorem below, which is a slightly modified version of Theorem 3.2 , confirms that the negative feedback interconnection of three systems, shown in Figure 3.2 , is passive if and only if all of the systems are passive. 


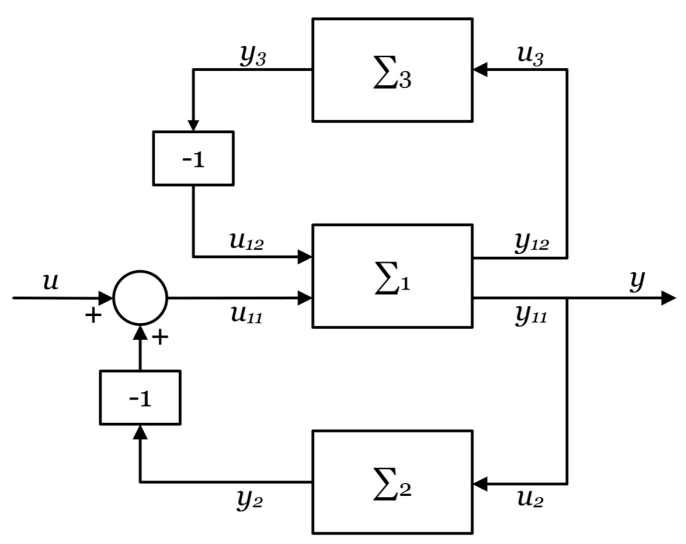

Figure 3.2: Feedback interconnection of three systems of form (3.1).

Theorem 3.3. Consider the feedback interconnection of three time-invariant dynamical systems of form (3.1). The closed-loop system shown in Figure 3.2 is strictly passive if all of the systems are strictly passive (or output strictly passive). Furthermore, for $u=0$ the origin of the system is asymptotically stable.

Proof: Consider the negative feedback interconnection of three systems $\Sigma_{1}, \Sigma_{2}$ and $\Sigma_{3}$ in the form of

$$
\Sigma_{i}:\left\{\begin{array}{l}
\dot{x_{i}}=f_{i}\left(x_{i}, u_{i}\right) \\
y_{i}=h_{i}\left(x_{i}, u_{i}\right)
\end{array} \quad, \quad i=1,2,3,\right.
$$

where for $\Sigma_{1}$, as shown in Figure 3.2, $u_{1}=\left[u_{11}, u_{12}\right]^{T}$ and $y_{1}=\left[y_{11}, y_{12}\right]^{T}$. Let $L_{1}\left(x_{1}\right)$, $L_{2}\left(x_{2}\right)$ and $L_{3}\left(x_{3}\right)$ be the positive definite storage functions for $\Sigma_{1}, \Sigma_{2}$ and $\Sigma_{3}$, respectively. Consider the positive definite storage function $L(x)=L_{1}\left(x_{1}\right)+L_{2}\left(x_{2}\right)+L_{3}\left(x_{3}\right)$ for the feedback interconnection system. Without loss of generality, consider that $\Sigma_{1}$ and $\Sigma_{2}$ are strictly passive and $\Sigma_{3}$ is output strictly passive, i.e., there exist functions $\varphi_{1}, \varphi_{2}$ and $\varphi_{3}$ such that $\varphi_{1}\left(x_{1}\right)>0, \varphi_{2}\left(x_{2}\right)>0$ and $y_{3}^{T} \varphi_{3}\left(y_{3}\right)>0, \forall x_{1}, x_{2}, y_{3} \neq 0$. So we get

$$
\dot{L}(x, u)=\dot{L_{1}}+\dot{L_{2}}+\dot{L_{3}} \leqslant u_{1}^{T} y_{1}+u_{2}^{T} y_{2}+u_{3}^{T} y_{3}-\varphi_{1}\left(x_{1}\right)-\varphi_{2}\left(x_{2}\right)-y_{3}^{T} \varphi_{3}\left(y_{3}\right) .
$$


From Figure 3.2 , we can see that $u_{11}=u-y_{2}, u_{12}=-y_{3}, u_{2}=y_{11}=y$ and $u_{3}=y_{12}$. Therefore, we have

$$
\begin{aligned}
\dot{L}(x, u) & \leqslant u_{11}^{T} y_{11}+u_{12}^{T} y_{12}+u_{2}^{T} y_{2}+u_{3}^{T} y_{3}-\varphi_{1}\left(x_{1}\right)-\varphi_{2}\left(x_{2}\right)-y_{3}^{T} \varphi_{3}\left(y_{3}\right) \\
& =\left(u-y_{2}\right)^{T} y_{11}-y_{3}^{T} y_{12}+y_{11}^{T} y_{2}+y_{12}^{T} y_{3}-\varphi_{1}\left(x_{1}\right)-\varphi_{2}\left(x_{2}\right)-y_{3}^{T} \varphi_{3}\left(y_{3}\right) \\
& =u^{T} y-\varphi\left(x_{1}, x_{2}, y_{3}\right),
\end{aligned}
$$

where $\varphi\left(x_{1}, x_{2}, y_{3}\right)=-\varphi_{1}\left(x_{1}\right)-\varphi_{2}\left(x_{2}\right)-y_{3}^{T} \varphi_{3}\left(y_{3}\right)$ and $y_{3}=h_{3}\left(x_{3}, u_{3}\right)$. Thus, $\dot{L}(x, u) \leqslant$ $u^{T} y-\varphi\left(x_{1}, x_{2}, x_{3}\right)$ and the feedback interconnection of the three systems is strictly passive. Now if we let $u=0$, we will get

$$
\dot{L}(x, 0) \leqslant-\varphi\left(x_{1}, x_{2}, x_{3}\right)<0 .
$$

So, by Lemma 3.1, it can be concluded that the origin of the feedback interconnected system is asymptotically stable.

Remark 3.2. Also in this system, since $\Sigma_{3}$ is output strictly passive, even if one of the systems has shortage of passivity, it could be compensated by the excess passivity of $\Sigma_{3}$. Therefore, it is guaranteed that the interconnection remains passive.

In this section and Section 3.1, we discussed passivity and stability analysis of the systems described by the state space model in equation (3.1). In the next section, we provide another definition of passivity for LTI systems.

\subsection{Positive Real Transfer Functions}

In this section, passivity of LTI systems in terms of transfer functions is discussed. Since finding storage functions is not an easy task, analyzing transfer functions of the LTI 
systems is more desirable for characterizing the passivity. For that purpose, consider the following definition and theorem adapted from [25].

Definition 3.2 ([25], Definition 6.4, p.237). A proper transfer function $G(s)$ of a SISO LTI system is called positive real if

- poles of $G(s)$ are in $\operatorname{Re}[s] \leqslant 0$,

- for all real $\omega$ for which $j \omega$ is not a pole of $G(s)$, the real part of $G(j \omega)$ is positive semi-definite, and

- any pure imaginary pole $j \omega$ is a simple pole and the residue transfer function $\lim _{s \rightarrow j \omega}(s-j \omega) G(s)$ is positive semi-definite Hermitian.

The transfer function $G(s)$ is called strictly positive real if $G(s-\varepsilon)$ is positive real for some $\varepsilon>0$.

Lemma 3.2 ([25], Lemma 6.4, p.241). The linear time-invariant system

$$
\begin{aligned}
& \dot{x}=A x+B u, \\
& y=C x+D u,
\end{aligned}
$$

with $G(s)=C(s I-A)^{-1} B+D$ is

- passive if $G(s)$ is positive real;

- strictly passive if $G(s)$ is strictly positive real.

Proof: See [25], Lemma 6.4.

\subsection{Summary}

In this chapter, we considered stability and passivity of dynamical systems as in equation (3.1). Also analysis of the negative feedback interconnection of these systems was 
discussed. After the background presented in Chapter 2 and Chapter 3 , now we turn to the main problem of this thesis. In the next chapter, an application of game theory to the water distribution system is presented. 


\section{Part II}

\section{Modeling and Stability Analysis}




\section{Chapter 4}

\section{Water Distribution System -}

\section{Modeling/Control Problem}

In this chapter, the application problem considered in this thesis is presented. The Water Distribution System (WDS) as an example of a resource allocation problem is introduced and a solution approach to the problem is suggested. This chapter is organized as follows. A motivation and the problem statement as well as a solution to the problem are provided in Sections 4.1-4.3. In Section 4.4, detailed formulation of the problem along with the standard notation is considered. Key contributions and materials are mainly taken from [17, 34, 36] and slightly modified where needed.

\subsection{Preview - Motivation}

Traditionally thinking, we consider population games as memoryless mappings from pop-

ulation state $p$ to payoffs $f(p)$ where the payoffs are static functions of strategy distribution. From this viewpoint, agents can obtain their payoffs by substituting their current strategy selections in the payoff functions and by utilizing an appropriate revision protocol, they can form the corresponding evolutionary dynamic in order to analyze the evolution of the game they are playing. 
As a well-behaved example of memoryless games, we can consider stable games (see Section 2.2.4), where the corresponding evolutionary dynamic, under certain conditions, converges to the $N E$ 's of the game. For instance, in [20, 35, 36], authors considered the EPT and the Smith dynamics and used the Lyapunov stability theorem to characterize the convergence and stability of the set of Nash equilibrium points of the stable games. It is demonstrated that the zero level set of the utilized Lyapunov function coincides with the set of the Nash equilibria of the game.

On the other hand, the authors of [17, 18] modeled evolutionary dynamics and the underlying games as input-output systems and connected them in the form of a negative feedback interconnection, shown in Figure 2.3 and 2.4, in order to employ passivity for characterizing the behavior of the game. In this setup, memoryless games are placed in $\Sigma_{1}$ and the evolutionary dynamics play the role of $\Sigma_{2}$ in the feedback loop. Then the authors proved that for zero input, a strictly passive interconnection will converge to the $N E$ 's of the underlying game. For a thorough discussion, the reader can refer to [36. Sections 7.1 and 7.2, p.237-264] where a connection between the zero level set of Lyapunov function and $N E$ 's of games has been established.

The idea in this thesis, is to extend these results to a more general class of games. As an extension from static population games, we assume that agents consider the games that have dynamics which means that the games are being played through dynamical systems. Here, we consider that evolutionary dynamics act on dynamically modified payoffs instead of static payoff functions. This modification can be interpreted as a coupling between a set of evolutionary dynamics and a game with dynamic dependencies.

As a result of this modification, we need to introduce new auxiliary states, other than the state vector of the mean dynamic reviewed in Chapter 2 , that capture the role of dynamically modified game. In this setup, dynamically modified games are mappings from strategy trajectories $p(t)$ to payoff trajectories $f(t)$. The figure below demonstrates the new configuration of evolutionary dynamics connected to dynamically modified games: 


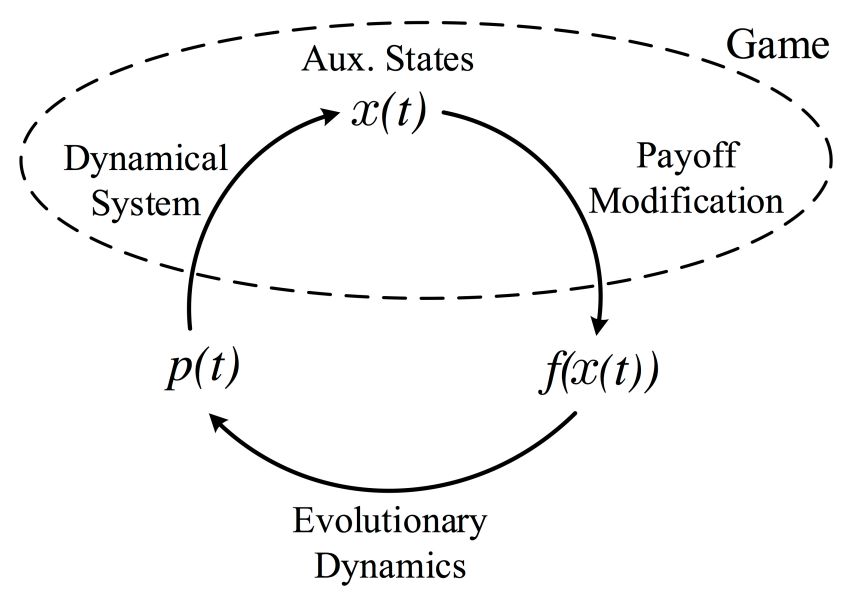

Figure 4.1: Connection of dynamical system of games and evolutionary dynamics.

where $x(t)$ and $f(x(t))$ are the state vector and the payoff vector of the dynamically modified game, respectively. In other words, modified games can be written as a dynamical system of form

$$
\dot{x}=F(x, p),
$$

where the function $F(x, p): \mathbb{R}^{N} \times \Delta \longrightarrow \mathbb{R}^{N}$ describes the dynamical model of the modified games. As can be seen, new payoffs are not necessarily functions of population state, i.e., agents gain auxiliary states of the dynamical system as a solution ${ }^{1}$ of equation (4.1) by playing the modified game and as a consequence, they need to define their new payoffs with respect to the dynamics of the game. Then they utilize a revision protocol that leads them to the standard evolutionary dynamic of the game they are playing.

As a typical example application, we consider the water distribution system. A WDS that consists of a number of storage tanks is employed to supply water to its costumers who need a certain amount and quality of service. We assume that one tank is available to each consumer. Based on demands of the consumers, each tank should have different characteristics to meet the expectations. Each consumer requires a specific amount of water stored in his tank and a certain pressure at his tank output. Therefore, in order to provide the pressure and the amount of needed water, we have to control the volume of

\footnotetext{
${ }^{1}$ To guarantee existence and uniqueness of the solution to equation 4.1 , it is required that $F(x, p)$ be Lipschitz continuous.
} 
water in each tank. We presume that there exists a total input flow of water as a resource to feed the tanks and fill them with water with respect to costumers' needs. Thus, if we assign a certain portion of the total input flow to each tank, we can control the amount of water in the tanks. This means that we are facing a resource allocation problem where the resource is total input flow of water that has to be allocated to the storage tanks.

We consider that a large number of artificial agents or players are supposed to take the responsibility of filling the tanks by allocating a certain amount of the resource to each tank. As the action set available to the population of agents, we assume that each tank is a possible choice for each agent. Therefore, if a player picks a tank, he dedicates a small portion of the resource to fill that tank. Thus, the more players choose a tank as their strategies, the more the selected tank is filled. This means that a WDS is considered as a population game that artificial players are to pick the tanks to fill as their strategies.

To formulate the allocation problem as a population game, we also need to assign a payoff to each tank, so agents can be attracted to fill the tanks. Then, by using a revision protocol, agents can model the evolution in time of the game they are playing as evolutionary dynamic in order to discuss the game play. Furthermore, since volume of the tanks varies with respect to time - because of time-varying input and output flows - a dynamical model is needed to describe the behavior of the tank system. So we can utilize the setup of Figure 4.1 where the tank system is the game with a dynamical system and the corresponding evolutionary dynamic is a controller coupled with the game to control its behavior.

In the next section, a detailed model of the tank system described in the form of a game as well as the corresponding evolutionary dynamic acting as a controller is provided. 


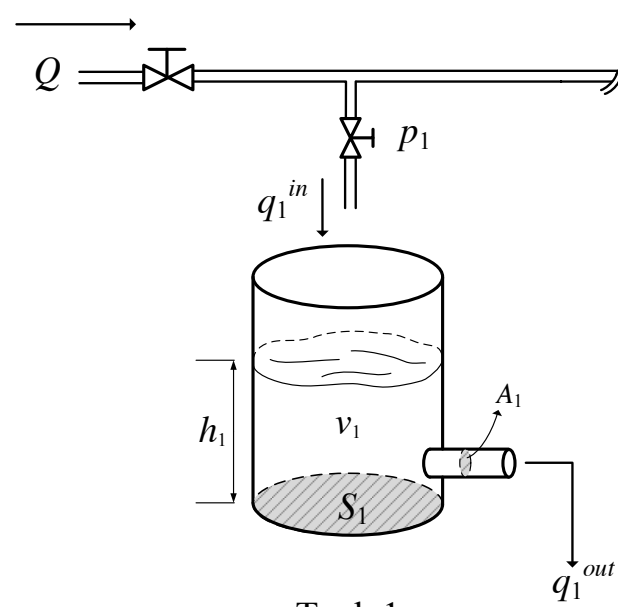

Tank 1

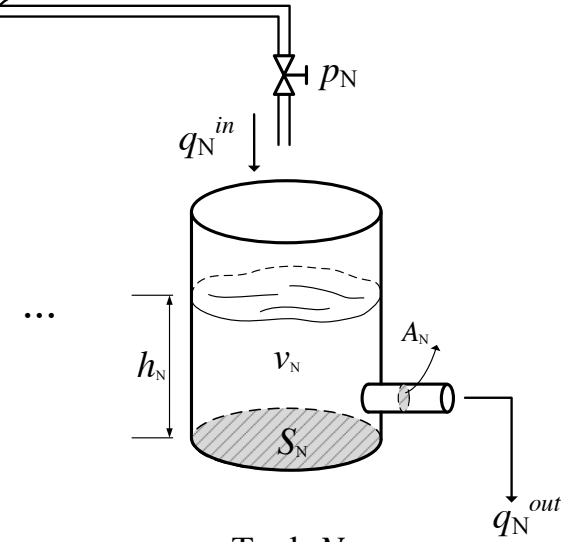

Tank $N$

Figure 4.2: Water Distribution System.

\subsection{Water Distribution System; $N$-Tank System}

In this section, we describe the model of a water distribution system. A WDS is used to supply water to all of its consumers and meet their demands. For this purpose, we consider a separate tank for each consumer. Based on needed pressure, capacity and the amount of water required for each consumer, tanks have different characteristics which should be considered in our model. Suppose that there is a set of $N$ different tanks for $N$ consumers in the system where a total available flow of water, denoted by $Q$, should be allocated to the tanks. We denote the input flow and the output flow of the $i$-th tank by $q_{i}^{\text {in }}$ and $q_{i}^{\text {out }}$, respectively. So the change of volume in each tank with respect to time is simply given by

$$
\frac{d v_{i}}{d t}=q_{i}^{i n}-q_{i}^{\text {out }}
$$

where $v_{i}$ is the volume of the $i$-th tank and $i=1,2, \ldots, N$.

As described in the previous section and shown in Figure 4.1, we propose to control the WDS via evolutionary dynamics as a centralized controller, which means that we have to formulate the WDS as a population game with dynamic dependencies as well as a controller based on the mean dynamic of the corresponding game under a given revision 
protocol.

In view of controlling the WDS by evolutionary dynamic, we assume a large number agents, who are employed to play the WDS game, have to allocate a certain amount of the total input flow of water $Q$ to each tank. So we consider each tank as a strategy for the players, i.e., agents choose different tanks to fill with water as their available set of strategies in this game, denoted $S=\{1, \ldots, N\}$. Also we assume that each tank is equipped with a control valve at its input pipe to be able to limit the flow of water. Since $p_{i}$ 's, which are the fractions of agents selecting the tanks, are proportions between 0 and 1 or from $0 \%$ to $100 \%$, they can be used as gates for the control valves to determine the percentage of how open or close the valves are to let water in the tanks. Therefore, $Q p_{i}$ will be the share of $i$-th tank from the total input water, i.e.,

$$
q_{i}^{i n}=Q p_{i}
$$

Now to model the output flow, for simplicity we assume that the tanks are being drained over the atmospheric pressure (gravity tank system [6, 34]), so the output flow, $q_{i}^{\text {out }}$, depends on the height of water stored in the tank and the transversal area of the output pipe, denoted by $h_{i}$ and $A_{i}$, respectively. Therefore, we can write the equation of output flow of the $i$-th tank as follows:

$$
q_{i}^{\text {out }}=A_{i} C_{i} \sqrt{2 g h_{i}}
$$

where $g$ is the acceleration due to the gravity and $C_{i}$ is the loss coefficient. If we assume that the transversal area of each tank, denoted by $S_{i}$, is constant and independent of $h_{i}$, we will have $v_{i}=h_{i} S_{i}$. So the equation of the output flow with respect to the volume of water in each tank can be rewritten as

$$
q_{i}^{\text {out }}=A_{i} C_{i} \sqrt{\frac{2 g v_{i}}{S_{i}}}
$$


Therefore, by using equations (4.2), (4.3) and (4.5), differential equations of the dynamical model of the WDS are given by

$$
\dot{v}_{i}=Q p_{i}-c_{i} \sqrt{v_{i}}
$$

where $c_{i}=A_{i} C_{i} \sqrt{2 g / S_{i}}$ is a parameter for each tank that is dependent on its output pipe and transversal area.

Now, we turn to controlling the $N$-tank system. In the next section, we introduce the Replicator Dynamic as a specified evolutionary dynamic to be the main controller of the WDS along with a secondary subsystem to dynamically control the total input flow.

\subsection{System Controllers}

According to the allocation problem and the suggested solution approach described in the previous sections, we consider designing a controller based on evolutionary dynamic to limit the input flow of each tank. Also we add another system to control the total input flow of the system $Q$. In the next parts, details of these systems are provided.

\subsubsection{Individual Input Flow Control}

As explained in Section 4.1, in order to analyze the behavior of the WDS as a game, we need to define payoff functions for the available strategies to get suitable controller based on evolutionary dynamic.

Recalling $v_{i}$ as the volume of water in the tanks, we use the following logistic type function (see [10]), which is proposed in [34]:

$$
f_{i}=-\frac{r_{i}}{v_{m_{i}}} v_{i}+r_{i}, \quad i=1, \ldots, N
$$

where $v_{m_{i}}$ is the maximum possible level of water in the $i$-th tank and $r_{i}$ is the maximum 
payoff that each strategy can get. In this configuration, the payoff function decreases as the volume of each tank gets closer to its maximum level, therefore, empty tanks are more attractive for the agents who are playing this game, i.e., more agents tend to play empty tanks. To model this behavior by differential equations of the Replicator Dynamic, first we calculate the average payoff according to the payoff functions introduced in equation (4.7)

$$
\bar{f}=\sum_{i=1}^{N} f_{i} p_{i}=\sum_{i=1}^{N} p_{i}\left(-\frac{r_{i}}{v_{m_{i}}} v_{i}+r_{i}\right)
$$

Now by using the proportional imitative revision protocol, shown in equation (2.9), we obtain the simplest evolutionary dynamic, the Replicator Dynamic, introduced in 2.11, as follows:

$$
\dot{p}_{i}=p_{i}\left(-\frac{r_{i}}{v_{m_{i}}} v_{i}+r_{i}-\sum_{j=1}^{N} p_{j}\left(-\frac{r_{j}}{v_{m_{j}}} v_{j}+r_{j}\right)\right)
$$

For simplicity, it is assumed that the maximum levels and the maximum payoffs of all tanks are the same, i.e., $v_{m_{i}}=v_{m}$ and $r_{i}=r$. So the Replicator equations become

$$
\dot{p}_{i}=-\frac{r}{v_{m}} p_{i}\left(v_{i}-\sum_{j=1}^{N} p_{j} v_{j}\right)
$$

Thus, by using this type of payoff function, the evolutionary dynamic will allocate less (more) resource to the tanks that have the payoff below (above) the average.

The Replicator Dynamic has to be interconnected to the $N$-tank system to control the input flow of each tank. But before illustrating this interconnection, we consider another subsystem that limits the total input flow of the WDS with respect to consumers' demands. Details of the secondary controller are provided in the next section.

\subsubsection{Total Input Flow Control}

In this section, we seek a model to dynamically control the total input flow of the system, $Q$, based on the consumers' demands and the desirable level of water in the tanks. This 
problem can be solved by the gradient descent method (see Appendix B.1).

In this problem, the objective is to minimize the consumption of water, so if we control the total input flow and, as a consequence, control the output flow of tanks, the goal is achieved. Therefore, controlling the consumption of water in the WDS turns into the problem of resource allocation optimization. We define a cost function with respect to $Q$ in order to control the total input flow and to maintain a satisfactory level of water in the tanks.

To minimize the consumption, the cost function is written as the difference between a convex pricing function $\mathcal{P}: \mathbb{R} \rightarrow \mathbb{R}$ and a strictly concave utility function $\mathcal{U}: \mathbb{R} \rightarrow \mathbb{R}$ in the following form:

$$
\mathcal{J}=\mathcal{P}-\mathcal{U}
$$

so the cost function is strictly convex. To define an appropriate utility function for our system, it is assumed that there is a limit on the total input flow for the long term run of the system. To clarify, we assume that instant overloads of $Q$ are allowed in the total input flow system but persistent overusages may cause damage to the system. So suppose $\hat{Q}$ is the maximum possible flow of water as the resource. Furthermore, clearly the input flow cannot be negative, hence

$$
0 \leqslant Q \leqslant \hat{Q}
$$

is the feasible interval of $Q$. So we define a utility function in the form of

$$
\mathcal{U}(Q)=\alpha\left(Q-\frac{Q^{2}}{2 \hat{Q}}\right)
$$

where $\alpha$ is a positive constant parameter. With this type of function, not only the property of strict concavity is satisfied, but also the desired maximum point of this function, which makes it positive, could be chosen in the desired interval, while the mentioned limit is not violated.

For the pricing function, we presume that the more input $(Q)$ the system gets, the 
higher the price will be. Also, we penalize having higher volume of water in the tanks. With this logic, we define the pricing function as follows:

$$
\mathcal{P}\left(Q, v_{1}, \ldots, v_{N}\right)=Q\left(\gamma+\beta \sum_{j=1}^{N} v_{j}\right),
$$

where $\gamma$ and $\beta$ are positive constant parameters and $v_{j}$ 's are the volumes of the tanks. Therefore, the minimization problem takes the form of

$$
\underset{Q}{\operatorname{minimize}} \mathcal{J}=Q\left(\gamma-\alpha+\beta \sum_{j=1}^{N} v_{j}\right)+\frac{\alpha}{2 \hat{Q}} Q^{2},
$$

where $\alpha$ and $\gamma$ are chosen such that $\alpha>\gamma$. We consider adjustments based on using negative gradient with respect to $Q$, i.e.,

$$
\dot{Q}=-k \frac{d \mathcal{J}}{d Q}=k\left(\alpha\left(1-\frac{Q}{\hat{Q}}\right)-\gamma-\beta \sum_{j=1}^{N} v_{j}\right)
$$

where $k>0$ is a constant (See Appendix B.1).

Together, the dynamical system of equation (4.16) and the evolutionary dynamic based controller, introduced in the previous section, control different parts of the WDS simultaneously to deliver a desirable behavior of the $N$-tank system to the consumers.

Next, we will combine the dynamics shown by equations (4.6), 4.10) and (4.16), to interconnect the water distribution system to the controllers.

\subsection{Feedback Interconnection - Standard Notation}

Consider the negative feedback interconnection of the tank system, the Replicator Dynamic and the total input flow controller in the form of Figure 4.3 , where the components $\Sigma_{1}, \Sigma_{2}$ and $\Sigma_{3}$ are identified as the tank system, the Replicator Dynamic and the total 


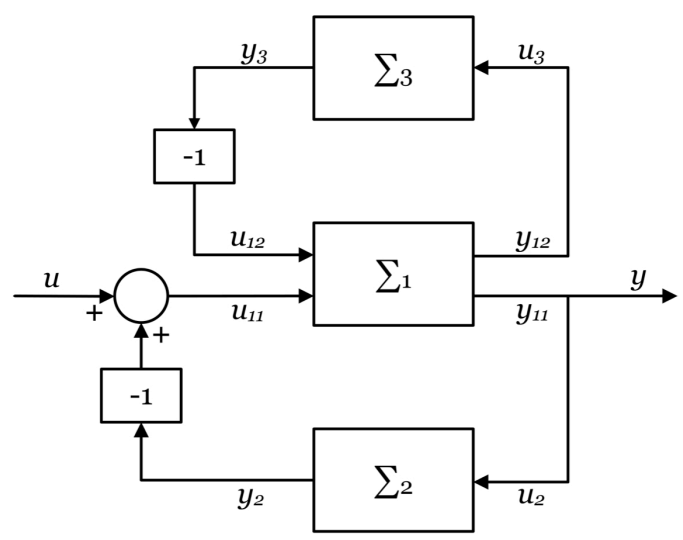

Figure 4.3: Feedback interconnection of the $N$-tank system with the evolutionary dynamic and the total input flow controllers.

input flow controller and are characterized by the state variables of $v=\left[v_{1}, \ldots, v_{N}\right]^{T}$ the volume of water in tanks, $p=\left[p_{1}, \ldots, p_{N}\right]^{T}$ the population state of the Replicator Dynamics and $Q$ the total input flow of the system, respectively. The outputs are $y_{1}=\left[y_{11}\right.$, $\left.y_{12}\right]^{T}=\left[v_{1}, \ldots, v_{N}, \Sigma_{i=1}^{N} v_{i}\right]^{T}, y_{2}=\left[-p_{1}, \ldots,-p_{N}\right]^{T}$ and $y_{3}=-Q$ and the external input of the system is $u$ which is ignored for now in deriving the system equations. From equations (4.6), (4.10) and 4.16), we get the interconnected system as follows:

$$
\begin{aligned}
& \Sigma_{1}:\left\{\begin{array}{l}
\dot{v}_{i}=Q p_{i}-c_{i} \sqrt{v_{i}} \\
y_{11 i}=v_{i}, \\
y_{12}=\sum_{j=1}^{N} v_{j}, \quad u_{12}=Q
\end{array}\right. \\
& \Sigma_{2}:\left\{\begin{array}{l}
\dot{p}_{i}=-\frac{r}{v_{m}} p_{i}\left(v_{i}-\sum_{j=1}^{N} p_{j} v_{j}\right) \\
y_{2 i}=-p_{i}, \quad u_{2 i}=v_{i}
\end{array} \quad i=1, \ldots, N .\right. \\
& \Sigma_{3}:\left\{\begin{array}{l}
\dot{Q}=k\left(\alpha\left(1-\frac{Q}{\hat{Q}}\right)-\gamma-\beta \sum_{j=1}^{N} v_{j}\right) \\
y_{3}=-Q, \quad u_{3}=\sum_{j=1}^{N} v_{j}
\end{array}\right.
\end{aligned}
$$

To calculate the equilibrium points $v_{i}^{*}, p_{i}^{*}$ and $Q^{*}$ for the zero input interconnected system 
$u=0$, which are found through an online optimization, we let the RHS of the differential equations, shown above, be zero:

$$
\begin{aligned}
k\left(\alpha\left(1-\frac{Q^{*}}{\hat{Q}}\right)-\gamma-\beta \sum_{j=1}^{N} v_{j}^{*}\right) & =0, \\
-\frac{r}{v_{m}} p_{i}^{*}\left(v_{i}^{*}-\sum_{j=1}^{N} p_{j}^{*} v_{j}^{*}\right) & =0, \\
Q^{*} p_{i}^{*}-c_{i} \sqrt{v_{i}^{*}} & =0 .
\end{aligned}
$$

From equation (4.19), for $p_{i}^{*} \neq 0$, we can simply see that the $v_{i}^{*}$ 's are equal since

$$
v_{i}^{*}-\sum_{j=1}^{N} v_{j}^{*} p_{j}^{*}=0 \Rightarrow v_{i}^{*}=\sum_{j=1}^{N} v_{j}^{*} p_{j}^{*}=v^{*}
$$

By summing the left hand side of equation 4.20 over $i$, we get

$$
Q^{*} \sum_{i=1}^{N} p_{i}^{*}-\sqrt{v^{*}} \sum_{i=1}^{N} c_{i}=0 \Rightarrow Q^{*}=C \sqrt{v^{*}}
$$

where $C=\sum_{i=1}^{N} c_{i}$. Also by substituting $Q^{*}$ in 4.20 , we can get $p_{i}^{*}$ as

$$
p_{i}^{*}=\frac{c_{i}}{C}
$$

Now by solving equation 4.18 and (4.22) for $Q^{*}$ and $v^{*}$, the solutions will be in the following form:

$$
\begin{aligned}
v^{*} & =\left(\frac{Q^{*}}{C}\right)^{2}, \\
Q^{*} & \left.=-\frac{\alpha C^{2}}{2 \beta N \hat{Q}}+C \sqrt{\left(\frac{\alpha C}{2 \beta N \hat{Q}}\right)^{2}+\frac{\alpha-\gamma}{\beta N}}>0 \quad \text { (Since } \alpha>\gamma\right), \\
Q^{*} & =-\frac{\alpha C^{2}}{2 \beta N \hat{Q}}-C \sqrt{\left(\frac{\alpha C}{2 \beta N \hat{Q}}\right)^{2}+\frac{\alpha-\gamma}{\beta N}}<0 \quad \text { (Not Acceptable). }
\end{aligned}
$$


Regarding the feasible interval of $Q$, shown in equation (4.12), around the equilibrium points $Q^{*}$ and $v_{j}^{*}$ s, we let the RHS of equation 4.16 be zero, so we obtain

$$
Q^{*}=\frac{\alpha-\gamma-\beta \sum_{j} v_{j}^{*}}{\alpha} \hat{Q}
$$

Since $\alpha, \gamma, \beta$ and $v_{j}^{*}$ s are all positive, if we choose the parameters and the desired equilibrium points in a way such that

$$
0 \leqslant \frac{\alpha-\gamma-\beta \sum_{j} v_{j}^{*}}{\alpha} \leqslant 1
$$

we get

$$
0 \leqslant Q^{*} \leqslant \hat{Q}
$$

which admits that the condition for $Q$, equation 4.12 , is satisfied for the equilibria.

For convenience in analyzing the system equations in the next chapters, we transform the current coordinate to error coordinates in standard notation. We write the equations in the standard form by letting

$$
\begin{aligned}
v=\left[v_{1}, \ldots, v_{N}\right]^{T} & =\left[x_{11}, \ldots, x_{1 N}\right]^{T}=x_{1}, \\
p=\left[p_{1}, \ldots, p_{N}\right]^{T} & =\left[x_{21}, \ldots, x_{2 N}\right]^{T}=x_{2}, \\
Q & =x_{3}, \\
v^{*}=x_{1}^{*}, \quad p^{*} & =x_{2}^{*}, \quad Q^{*}=x_{3}^{*} .
\end{aligned}
$$

To obtain the equations in the error coordinates, by considering equation (4.25), let

$$
\begin{aligned}
\Delta x_{1}=\left[\Delta x_{11}, \ldots, \Delta x_{1 N}\right]^{T}=x_{1}-x_{1}^{*} \mathbf{1} & =v-v^{*} \mathbf{1}=\left[v_{1}-v^{*}, \ldots, v_{N}-v^{*}\right]^{T}, \\
\Delta x_{2}=\left[\Delta x_{21}, \ldots, \Delta x_{2 N}\right]^{T}=x_{2}-x_{2}^{*} & =p-p^{*}=\left[p_{1}-p_{1}^{*}, \ldots, p_{N}-p_{N}^{*}\right]^{T}, \\
\Delta x_{3}=x_{3}-x_{3}^{*} & =Q-Q^{*}
\end{aligned}
$$


where $\Delta x_{1} \in \mathbb{R}^{N}, \Delta x_{2} \in \mathbb{R}^{N}, \Delta x_{3} \in \mathbb{R}$ and $\mathbf{1}=[1, \ldots, 1]^{T} \in \mathbb{R}^{N}$. Now with the new notations, the system equations become

$$
\begin{aligned}
& \Sigma_{1}:\left\{\begin{array}{l}
\Delta \dot{x}_{1 i}=\left(\Delta x_{3}+x_{3}^{*}\right)\left(\Delta x_{2 i}+x_{2 i}^{*}\right)-c_{i} \sqrt{\Delta x_{1 i}+x_{1}^{*}} \\
y_{11_{i}}=\Delta x_{1 i}, \\
u_{11_{i}}=\Delta x_{2 i}=\sum_{j=1}^{N} \Delta x_{1 j}, \quad u_{12}=\Delta x_{3}
\end{array}\right. \\
& \Sigma_{2}:\left\{\begin{array}{c}
\Delta \dot{x}_{2 i}=-\frac{r}{v_{m}}\left(\Delta x_{2 i}+x_{2 i}^{*}\right)\left(\Delta x_{1 i}-\sum_{j=1}^{N} \Delta x_{1 j}\left(\Delta x_{2 j}+x_{2 j}^{*}\right)\right) \\
u_{2 i}=-\Delta x_{2 i}, \quad \Delta x_{1 i}
\end{array}\right. \\
& \Sigma_{3}:\left\{\begin{array}{cc}
\Delta \dot{x}_{3}=k\left(\alpha\left(1-\frac{\Delta x_{3}+x_{3}^{*}}{\hat{Q}}\right)-\gamma-\beta \sum_{j=1}^{N}\left(\Delta x_{1 j}+x_{1}^{*}\right)\right) \\
y_{3}=-\Delta x_{3},
\end{array},\right.
\end{aligned}
$$

where $i=1, \ldots, N$. By rearranging equation (4.28), we get

$$
\Delta \dot{x}_{3}=k\left(-\frac{\alpha}{\hat{Q}} \Delta x_{3}-\beta \sum_{j=1}^{N} \Delta x_{1 j}\right)+k\left(\alpha\left(1-\frac{x_{3}^{*}}{\hat{Q}}\right)-\gamma-\beta \sum_{j=1}^{N} x_{1}^{*}\right)
$$

where the second term in the RHS of equation (4.29) is the equation of the equilibrium point 4.18 which is equal to zero, so only the first term remains in the equation of $\Sigma_{3}$, which gives a SISO LTI system

$$
\Sigma_{3}:\left\{\begin{array}{rl}
\Delta \dot{x}_{3} & =-\frac{k \alpha}{\hat{Q}} \Delta x_{3}-k \beta \sum_{j=1}^{N} \Delta x_{1 j} \\
u_{3} & =\sum_{j=1}^{N} \Delta x_{1 j} \\
y_{3} & =-\Delta x_{3}
\end{array} .\right.
$$

Due to the property of Lipschitz continuity as a requirement in mathematical analysis of dynamical systems and also physical restrictions of the WDS, we need to impose some 
limits on the corresponding differential equations of the system under study. If we consider $\Delta x=\left[\Delta x_{1}^{T}, \Delta x_{2}^{T}, \Delta x_{3}\right]^{T}$ as the state vector of the negative feedback interconnected system, we can define the following domain for the WDS and its controllers:

$$
\begin{aligned}
\mathcal{X}=\left\{\Delta x=\left[\Delta x_{1}^{T}, \Delta x_{2}^{T}, \Delta x_{3}\right]^{T}\right. & \in \mathbb{R}^{2 N+1}: \Delta x_{1 i}>-x_{1}^{*}, \\
\Delta x_{2}+x_{2}^{*} & \left.\in \mathbb{R}_{+}^{N}, \Delta x_{3}>-x_{3}^{*}, \quad \forall i=1, \ldots, N\right\},
\end{aligned}
$$

under which the physical interpretations of the equations for the WDS are meaningful and equations remain locally Lipschitz continuous. This domain remains the same in all parts of this thesis in the next sections.

In the next chapter, we consider a discussion about passivity and stability of the introduced systems. 


\section{Chapter 5}

\section{Stability Analysis}

In this chapter, we investigate the stability and convergence to the desired equilibria, derived in the previous section, of the water distribution system in connection with the two proposed controllers in Chapter 4. The objective is to show the stability of the negative feedback interconnected system using the passivity and the stability theorems presented in Chapter 2. In Section 5.1, first we characterize the WDS in connection with the Replicator Dynamic as the main controller. Then in Section 5.2, we discuss a potential problem due to the (NS) property that could be caused by using the Replicator Dynamic. Also we present a solution that improves this behavior and the stability characteristics of the interconnected system.

\subsection{The Replicator Dynamic}

In the previous chapter, we obtained the model of the interconnected three systems. The interconnected system consists of the WDS as the plant, the Replicator Dynamic as the main controller of the tank system and a secondary controller for the total input flow. In this section, we analyze the stability of the interconnected system. 


\subsubsection{Passivity of the Interconnected System}

Considering the WDS and the Replicator Dynamic introduced in Chapter 4, the theorem below investigates the convergence of the interconnected system to the desired equilibrium point.

Theorem 5.1. The negative feedback interconnection of $\Sigma_{1}, \Sigma_{2}$ and $\Sigma_{3}$, described by equations 4.26), 4.27) and 4.30, shown in Figure 4.3, is locally passive with a domain of $D \subset \mathcal{X}$. Furthermore, for $u=0$ the equilibrium point of the system is locally stable.

Proof: In this proof, Theorem 3.3 is applicable. However, we utilize another approach that only requires Theorem 3.2 . First, we prove the passivity of $\Sigma_{2}$ and $\Sigma_{3}$ and then to analyze the passivity of $\Sigma_{1}$, we combine the dynamics of $\Sigma_{1}$ and $\Sigma_{3}$. Consider $\Sigma_{2}$ and the following relative entropy type function as a storage function candidate

$$
L_{2}\left(\Delta x_{2}\right)=-\frac{v_{m}}{r} \sum_{j=1}^{N} x_{2 j}^{*} \ln \left(\frac{\Delta x_{2 j}+x_{2 j}^{*}}{x_{2 j}^{*}}\right)
$$

By applying Jensen's inequality to the ln function, we observe that

$$
L_{2}\left(\Delta x_{2}\right)=-\frac{v_{m}}{r} \sum_{j=1}^{N} x_{2 j}^{*} \ln \left(\frac{\Delta x_{2 j}+x_{2 j}^{*}}{x_{2 j}^{*}}\right) \geqslant-\frac{v_{m}}{r} \ln \left(\sum_{j=1}^{N} x_{2 j}^{*}\left(\frac{\Delta x_{2 j}+x_{2 j}^{*}}{x_{2 j}^{*}}\right)\right)=0,
$$

where $L_{2}\left(\Delta x_{2}\right)=0$ if and only if $\Delta x_{2}=0$. Thus, $L_{2}$ is a valid positive definite storage function and its derivative along the trajectories of the Replicator Dynamic, equation 4.27), is given by

$$
\begin{aligned}
\dot{L}_{2} & =-\frac{v_{m}}{r} \sum_{j=1}^{N} x_{2 j}^{*} \frac{\Delta \dot{x}_{2 j}}{\Delta x_{2 j}+x_{2 j}^{*}}=\sum_{j=1}^{N} x_{2 j}^{*}\left(\Delta x_{1 j}-\sum_{k=1}^{N} \Delta x_{1 k}\left(\Delta x_{2 k}+x_{2 k}^{*}\right)\right) \\
& =-\sum_{k=1}^{N} \Delta x_{1 k} \Delta x_{2 k}=-\left(\Delta x_{1}\right)^{T} \Delta x_{2}=u_{2}^{T} y_{2},
\end{aligned}
$$

which by Definition 3.1, shows that $\Sigma_{2}$ is lossless. 
Now consider the following positive definite storage function for $\Sigma_{3}$ :

$$
L_{3}\left(\Delta x_{3}\right)=\frac{1}{2 k \beta} \Delta x_{3}^{2}
$$

By taking the derivative along 4.30, we get

$$
\begin{aligned}
\dot{L}_{3} & =\frac{1}{k \beta} \Delta x_{3} \Delta \dot{x}_{3}=\frac{1}{k \beta} \Delta x_{3}\left(-\frac{k \alpha}{\hat{Q}} \Delta x_{3}-k \beta \sum_{j=1}^{N} \Delta x_{1 j}\right) \\
& =-\Delta x_{3} \sum_{j=1}^{N} \Delta x_{1 j}-\frac{\alpha}{\beta \hat{Q}} \Delta x_{3}^{2}=u_{3}^{T} y_{3}-\frac{\alpha}{\beta \hat{Q}} \Delta x_{3}^{2} .
\end{aligned}
$$

Clearly, by Definition 3.1, $\Sigma_{3}$ is output strictly passive. In the next part of the proof, first we prove that $\Sigma_{1}$ is passive from $u_{12}$ to $y_{12}$. For that purpose, consider the positive storage function $L_{12}$ as follows:

$$
L_{12}\left(\Delta x_{1}\right)=\frac{1}{2}\left(\sum_{j=1}^{N} \Delta x_{1 j}\right)^{2}
$$

By taking the derivative along (4.26), the result will be

$$
\begin{aligned}
\dot{L}_{12} & =\sum_{j=1}^{N} \Delta x_{1 j} \sum_{k=1}^{N} \Delta \dot{x}_{1 k} \\
& =\sum_{j=1}^{N} \Delta x_{1 j} \sum_{k=1}^{N}\left(\left(\Delta x_{3}+x_{3}^{*}\right)\left(\Delta x_{2 k}+x_{2 k}^{*}\right)-c_{k} \sqrt{\Delta x_{1 k}+x_{1}^{*}}\right) \\
& =\sum_{j=1}^{N} \Delta x_{1 j}\left(\Delta x_{3}+x_{3}^{*}-\sum_{k=1}^{N} c_{k} \sqrt{\Delta x_{1 k}+x_{1}^{*}}\right) \\
& =\Delta x_{3} \sum_{j=1}^{N} \Delta x_{1 j}+\sum_{j=1}^{N} \Delta x_{1 j}\left(x_{3}^{*}-\sum_{k=1}^{N} c_{k} \sqrt{\Delta x_{1 k}+x_{1}^{*}}\right) .
\end{aligned}
$$

Considering equation 4.22 in standard notations, we have $x_{3}^{*}=C \sqrt{x_{1}^{*}}=\sum_{k=1}^{N} c_{k} \sqrt{x_{1}^{*}}$. 
Therefore, we get

$$
\dot{L}_{12}=u_{12}^{T} y_{12}+\theta_{1}\left(\Delta x_{1}\right)
$$

where $\theta_{1}\left(\Delta x_{1}\right)=\sum_{j=1}^{N} \Delta x_{1 j} \sum_{k=1}^{N} c_{k}\left(\sqrt{x_{1}^{*}}-\sqrt{\Delta x_{1 k}+x_{1}^{*}}\right)$. There are three possible scenarios for $\theta_{1}\left(\Delta x_{1}\right)$ as follows:

1. $0 \leqslant x_{1 k}<x_{1}^{*}$ :

In this case, we have $\Delta x_{1 k}<0$, so $\sqrt{x_{1}^{*}}>\sqrt{\Delta x_{1 k}+x_{1}^{*}}$. Therefore, $\theta_{1}\left(\Delta x_{1}\right)<0$.

2. $x_{1 k}=x_{1}^{*}$ :

We have $\Delta x_{1 k}=0$, so $\theta_{1}\left(\Delta x_{1}\right)=0$.

3. $x_{1 k}>x_{1}^{*}$ :

Here we get $\Delta x_{1 k}>0$, then $\sqrt{x_{1}^{*}}<\sqrt{\Delta x_{1 k}+x_{1}^{*}}$. Thus, $\theta_{1}\left(\Delta x_{1}\right)<0$.

So with this negative definite function $\theta_{1}$, we get $\dot{L}_{12}=u_{12}^{T} y_{12}+\theta_{1}\left(\Delta x_{1}\right) \leqslant u_{12}^{T} y_{12}$. Thus, $\Sigma_{1}$ is strictly passive from the input $u_{12}$ to the output $y_{12}$. For the rest of the proof, some considerations are taken into account to complete the proof of the passivity of $\Sigma_{1}$.

We just proved that $\Sigma_{3}$ and the input-output pair $\left(u_{12}, y_{12}\right)$ of $\Sigma_{1}$ are both strictly passive, so their feedback interconnection, by Theorem 3.2 , is asymptotically stable. Then, without loss of generality, we concatenate $\Sigma_{3}$ and $\Sigma_{1}$ to get the augmented state vector of $\Delta x_{1}^{\prime}=\left[\Delta x_{11}, \ldots, \Delta x_{1 N}, \Delta x_{3}\right]^{T}$. Let us call the new system $\Sigma_{1}^{\prime}$ as shown in Figure 5.1. Now consider the following storage function for the new augmented system $\Sigma_{1}^{\prime}$ :

$$
\begin{aligned}
L_{1}^{\prime}\left(\Delta x_{1}^{\prime}\right) & =\frac{1}{2\left(\Delta x_{3}+x_{3}^{*}\right)} \sum_{j=1}^{N}\left(\Delta x_{1 j}\right)^{2}+d\left(L_{12}+L_{3}\right) \\
& =\frac{1}{2\left(\Delta x_{3}+x_{3}^{*}\right)} \sum_{j=1}^{N}\left(\Delta x_{1 j}\right)^{2}+\frac{d}{2}\left(\left(\sum_{j=1}^{N} \Delta x_{1 j}\right)^{2}+\frac{1}{k \beta} \Delta x_{3}^{2}\right)
\end{aligned}
$$

where $d>0$ is a constant. It is clear that $L_{1}^{\prime}\left(\Delta x_{1}^{\prime}\right)$ is non-negative and $L_{1}^{\prime}\left(\Delta x_{1}^{\prime}\right)=0$ if and only if $\Delta x_{1}^{\prime}=0$, i.e., $\Delta x_{1}=0$ and $\Delta x_{3}=0$. Therefore, it is a positive definite 


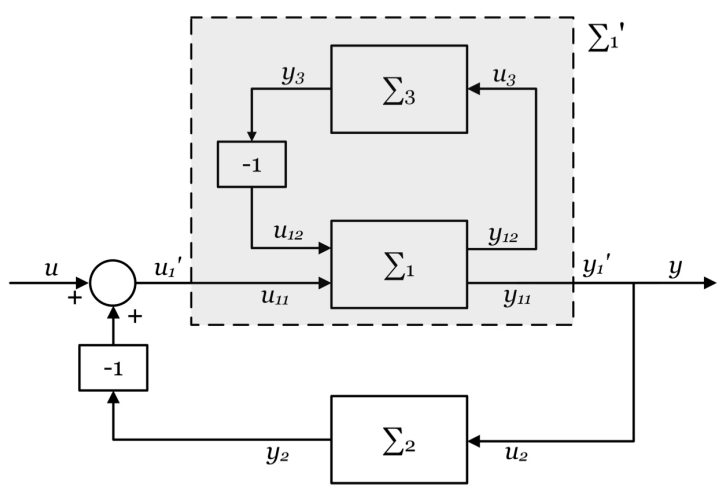

Figure 5.1: Feedback interconnection of the concatenated system $\Sigma_{1}^{\prime}$ and $\Sigma_{2}$.

storage function. The derivative along $(4.26$ and 4.30 will be the following:

$$
\begin{aligned}
\dot{L}_{1}^{\prime}= & \sum_{j=1}^{N} \frac{\partial L_{1}^{\prime}}{\partial \Delta x_{1 j}} \Delta \dot{x}_{1 j}+\frac{\partial L_{1}^{\prime}}{\partial \Delta x_{3}} \Delta \dot{x}_{3} \\
= & \frac{1}{\Delta x_{3}+x_{3}^{*}} \sum_{j=1}^{N} \Delta x_{1 j} \Delta \dot{x}_{1 j}-\frac{\Delta \dot{x}_{3}}{2\left(\Delta x_{3}+x_{3}^{*}\right)^{2}} \sum_{j=1}^{N}\left(\Delta x_{1 j}\right)^{2} \\
& +d\left(\sum_{j=1}^{N} \Delta x_{1 j} \sum_{k=1}^{N} \Delta \dot{x}_{1 k}+\frac{1}{k \beta}\left(\Delta x_{3} \Delta \dot{x}_{3}\right)\right)
\end{aligned}
$$

Now, by using equations (5.4) and (5.6) for the last term on the RHS of the equation above and also equations 4.26) and 4.30, we get

$$
\begin{aligned}
\dot{L}_{1}^{\prime}= & \sum_{j=1}^{N} \Delta x_{1 j}\left(\Delta x_{2 j}+x_{2 j}^{*}-\frac{1}{\Delta x_{3}+x_{3}^{*}} c_{j} \sqrt{\Delta x_{1 j}+x_{1}^{*}}\right)-\frac{\Delta \dot{x}_{3}}{2\left(\Delta x_{3}+x_{3}^{*}\right)^{2}} \sum_{j=1}^{N}\left(\Delta x_{1 j}\right)^{2} \\
& +d\left(\sum_{j=1}^{N} \Delta x_{1 j}\left(x_{3}^{*}-\sum_{k=1}^{N} c_{k} \sqrt{\Delta x_{1 k}+x_{1}^{*}}\right)-\frac{\alpha}{\beta \hat{Q}} \Delta x_{3}^{2}\right) .
\end{aligned}
$$

By rearranging the equations, we arrive at

$$
\begin{aligned}
\dot{L}_{1}^{\prime} & =\sum_{j=1}^{N} \Delta x_{1 j} \Delta x_{2 j}+\sum_{j=1}^{N} \Delta x_{1 j}\left(x_{2 j}^{*}-\frac{1}{\Delta x_{3}+x_{3}^{*}} c_{j} \sqrt{\Delta x_{1 j}+x_{1}^{*}}\right) \\
& -\frac{\Delta \dot{x}_{3}}{2\left(\Delta x_{3}+x_{3}^{*}\right)^{2}} \sum_{j=1}^{N}\left(\Delta x_{1 j}\right)^{2}+d\left(\sum_{j=1}^{N} \Delta x_{1 j}\left(x_{3}^{*}-\sum_{k=1}^{N} c_{k} \sqrt{\Delta x_{1 k}+x_{1}^{*}}\right)-\frac{\alpha}{\beta \hat{Q}} \Delta x_{3}^{2}\right) .
\end{aligned}
$$


From equation 4.26 and Figure 5.1, it is clear that $y_{1}^{\prime}=y_{11}=\left[\Delta x_{11}, \ldots, \Delta x_{1 N}\right]^{T}$ and $u_{1}^{\prime}=u_{11}=\left[\Delta x_{21}, \ldots, \Delta x_{2 N}\right]^{T}$. So we get

$$
\begin{aligned}
\dot{L}_{1}^{\prime}= & \left(u_{1}^{\prime}\right)^{T} y_{1}^{\prime}+\frac{1}{\Delta x_{3}+x_{3}^{*}} \sum_{j=1}^{N} \Delta x_{1 j}\left(x_{2 j}^{*} x_{3}^{*}-c_{j} \sqrt{\Delta x_{1 j}+x_{1}^{*}}\right)+\frac{\Delta x_{3}}{\Delta x_{3}+x_{3}^{*}} \sum_{j=1}^{N} \Delta x_{1 j} x_{2 j}^{*} \\
& -\frac{\Delta \dot{x}_{3}}{2\left(\Delta x_{3}+x_{3}^{*}\right)^{2}} \sum_{j=1}^{N}\left(\Delta x_{1 j}\right)^{2}+d\left(\sum_{j=1}^{N} \Delta x_{1 j}\left(x_{3}^{*}-\sum_{k=1}^{N} c_{k} \sqrt{\Delta x_{1 k}+x_{1}^{*}}\right)-\frac{\alpha}{\beta \hat{Q}} \Delta x_{3}^{2}\right) \\
= & \left(u_{1}^{\prime}\right)^{T} y_{1}^{\prime}+d\left(\theta_{1}+\theta_{2}\right)+\theta_{3}+\theta_{4},
\end{aligned}
$$

where

$$
\begin{aligned}
& \theta_{1}=\sum_{j=1}^{N} \Delta x_{1 j} \sum_{k=1}^{N} c_{k}\left(\sqrt{x_{1}^{*}}-\sqrt{\Delta x_{1 k}+x_{1}^{*}}\right) \\
& \theta_{2}=-\frac{\alpha}{\beta \hat{Q}} \Delta x_{3}^{2} \\
& \theta_{3}=\frac{1}{\Delta x_{3}+x_{3}^{*}} \sum_{j=1}^{N} \Delta x_{1 j}\left(x_{2 j}^{*} x_{3}^{*}-c_{j} \sqrt{\Delta x_{1 j}+x_{1}^{*}}\right), \\
& \theta_{4}=\frac{\Delta x_{3}}{\Delta x_{3}+x_{3}^{*}} \sum_{j=1}^{N} \Delta x_{1 j} x_{2 j}^{*}-\frac{\Delta \dot{x}_{3}}{2\left(\Delta x_{3}+x_{3}^{*}\right)^{2}} \sum_{j=1}^{N}\left(\Delta x_{1 j}\right)^{2} .
\end{aligned}
$$

Since $\theta_{1}$ is the same function shown in equation (5.7), it is strictly negative. Also, $\theta_{2}$ is clearly strictly negative. Therefore, for all $d>0, d\left(\theta_{1}+\theta_{2}\right)$ is strictly negative.

For $\theta_{3}$, by equations 4.22, 4.23 and 4.25 we can rewrite it to get the following form:

$$
\theta_{3}=\frac{1}{\Delta x_{3}+x_{3}^{*}} \sum_{j=1}^{N} \Delta x_{1 j} c_{j}\left(\sqrt{x_{1}^{*}}-\sqrt{\Delta x_{1 j}+x_{1}^{*}}\right) .
$$

Due to the feasible interval of $Q, \Delta x_{3}+x_{3}^{*}=x_{3}$ is positive, so with the same reasoning discussed for $\theta_{1}$ we can say that $\theta_{3}$ is also strictly negative. But for the last function, $\theta_{4}$, nothing can be stated about its sign, i.e., it could be either positive or negative. So recalling $\Delta x=\left[\Delta x_{1}^{T}, \Delta x_{2}^{T}, \Delta x_{3}\right]^{T}$ and considering the equations of $[5.10$, if

$$
\left(\exists d, \delta_{d}>0\right) \Delta x \in D_{\delta} \Longrightarrow\left|\theta_{4}\right|<\left|d\left(\theta_{1}+\theta_{2}\right)+\theta_{3}\right|
$$


where

$$
D_{\delta}=\left\{\Delta x=\left[\Delta x_{1}^{T}, \Delta x_{2}^{T}, \Delta x_{3}\right]^{T} \in \mathbb{R}^{2 N+1}:\|\Delta x\|_{2}<\delta_{d}\right\} \subset \mathcal{X}
$$

is called the Domain of Attraction (D.o.A), then

$$
d\left(\theta_{1}+\theta_{2}\right)+\theta_{3}+\theta_{4}<0
$$

and

$$
\dot{L}_{1}^{\prime} \leqslant\left(u_{1}^{\prime}\right)^{T} y_{1}^{\prime}-\varphi_{1}^{\prime}\left(\Delta x_{1}^{\prime}\right)
$$

where $\varphi_{1}^{\prime}\left(\Delta x_{1}^{\prime}\right)=d\left(\theta_{1}+\theta_{2}\right)+\theta_{3}+\theta_{4}<0$ and $\varphi_{1}^{\prime}(0)=0$. Hence, the concatenated system $\Sigma_{1}^{\prime}$ is strictly passive within $D_{\delta}$. Now, by considering the storage functions defined in equations (5.1) and (5.8), if we consider a storage function of form

$$
L=L_{1}^{\prime}+L_{2}
$$

for the negative feedback interconnection, shown in Figure 5.1, and take the derivative with respect to time, by Theorem 3.2 , we get

$$
\begin{aligned}
\dot{L}=\dot{L}_{1}^{\prime}+\dot{L}_{2} & \leqslant\left(u_{1}^{\prime}\right)^{T} y_{1}^{\prime}-\varphi_{1}^{\prime}\left(\Delta x_{1}^{\prime}\right)+u_{2}^{T} y_{2} \\
& =\left(u-y_{2}\right)^{T} y_{1}^{\prime}+\left(y_{1}^{\prime}\right)^{T} y_{2}-\varphi_{1}^{\prime}\left(\Delta x_{1}^{\prime}\right) \\
& =u^{T} y-\varphi_{1}^{\prime}\left(\Delta x_{1}^{\prime}\right) .
\end{aligned}
$$

This confirms that the negative feedback interconnection of Figure 5.1 is passive. However, the strict passivity of the whole system is not guaranteed, because the state vector of the Replicator Dynamics, $\Delta x_{2}$, does not appear in $\varphi_{1}^{\prime}\left(\Delta x_{1}^{\prime}\right)$. Therefore, nothing can be stated about the asymptotic stability of the Replicator Dynamic in converging to the desired equilibria $\left(\Delta x_{2}=0\right)$. Thus, the equilibrium point of the interconnected system is only stable (not asymptotically stable) for zero input $u=0$. 
Remark 5.1. As shown in the proof of Theorem 5.1, appropriate values of $d$ and $\delta_{d}$ are required for the system to admit the property of passivity. In Appendix A.3, criteria for choosing proper $d$ and $\delta_{d}$ and their effects on the system are characterized.

Remark 5.2. In [34, by a similar approach in the stability analysis of the WDS in connection to the Replicator Dynamic, it is shown that the Replicator Dynamic is lossless. Therefore, as explained in the proof of Theorem 5.1, the feedback interconnection of the WDS with the controller is not strictly passive and hence, asymptotic stability of the corresponding equilibrium is not guaranteed. In fact, under some conditions, the Replicator Dynamic fails in converging to the desired equilibrium points, so it makes the system not function properly. Thus, there is a need to improve the behavior of the controller of the WDS, which will be addressed in the next section.

In this section, we proved that the interconnected system is passive, so it is stable for the zero inputs. However, there exists a potential problem with the Replicator Dynamic that can cause undesirable convergence to non-Nash equilibrium points. In the next section, a solution to this problem is provided.

\subsection{Convergence to the Interior $N E$}

As we showed in the previous sections, the Replicator Dynamic as a controller for the water distribution system satisfies the passivity conditions and fits our problem framework. This model of the evolutionary dynamic is known to be the simplest to analyze and implement, however, it has a disadvantage which could cause some undesirable behaviors in the system. The problem occurs when the Replicator Dynamic admits non-Nash equilibrium points on the boundary of the simplex $\Delta$. For instance in our system, consider the equilibrium points given by equations 4.18, 4.19) and 4.20 and let $p_{j}^{*}=0$ for a $j \in S$. Clearly, by equation 4.19, $p_{j}^{*}$ is one of the equilibrium points of the Replicator 
Dynamic. Therefore, a set of the new equilibrium points will be the following:

$$
\left\{\begin{array}{l}
p_{j}^{*}=0 \Rightarrow v_{j}^{*}=0 \\
p_{i}^{*}=\frac{c_{i}}{\sum_{\substack{k=1 \\
k \neq j}}^{N} c_{k}} \Rightarrow v_{i}^{*}=\left(\frac{Q^{*}}{\sum_{k \neq j} c_{k}}\right)^{2}, \quad \begin{array}{l}
i=1, \ldots, N, \\
i \neq j
\end{array}
\end{array}\right.
$$

which is not desired because the $j$-th tank is empty. In Section 2.2 and in [36], it has been explained in detail that the Replicator Dynamic satisfies (PC) but fails (NS) so it can admit non-Nash equilibrium points. Therefore, to overcome this problem and to discard the undesirable equilibrium points, we utilize a new set of dynamics that satisfies both (PC) and (NS). As proved in Chapter 2, two dynamical models of population games that satisfy both conditions are the BNN dynamic and the Smith dynamic. In the next sections, these dynamics and their stability analysis are presented.

\subsubsection{The BNN Dynamic}

Considering the BNN dynamic shown in equation (2.21) and the payoff function defined in equation (4.7), in the standard notation, the BNN dynamic takes the form of

$$
\Delta \dot{x}_{2 i}=\left[\hat{f}_{i}\right]_{+}-\left(\Delta x_{2 i}+x_{2 i}^{*}\right) \sum_{j=1}^{N}\left[\hat{f}_{j}\right]_{+}
$$

where

$$
\hat{f}_{i}=\frac{r}{v_{m}}\left(\sum_{k=1}^{N}\left(\Delta x_{2 k}+x_{2 k}^{*}\right) \Delta x_{1 k}-\Delta x_{1 i}\right) .
$$

Now by putting equations 4.26, 4.30 and (5.17) together, we will have a new set of dynamics

$$
\underset{\text { Dynamic }}{\mathrm{BNN}}:\left\{\begin{array}{rl}
\Delta \dot{x}_{2 i} & =\left[\hat{f}_{i}\right]_{+}-\left(\Delta x_{2 i}+x_{2 i}^{*}\right) \sum_{j=1}^{N}\left[\hat{f}_{j}\right]_{+}, \\
y_{2 i} & =-\Delta x_{2 i}, \quad u_{2 i}=\Delta x_{1 i}
\end{array},\right.
$$


and

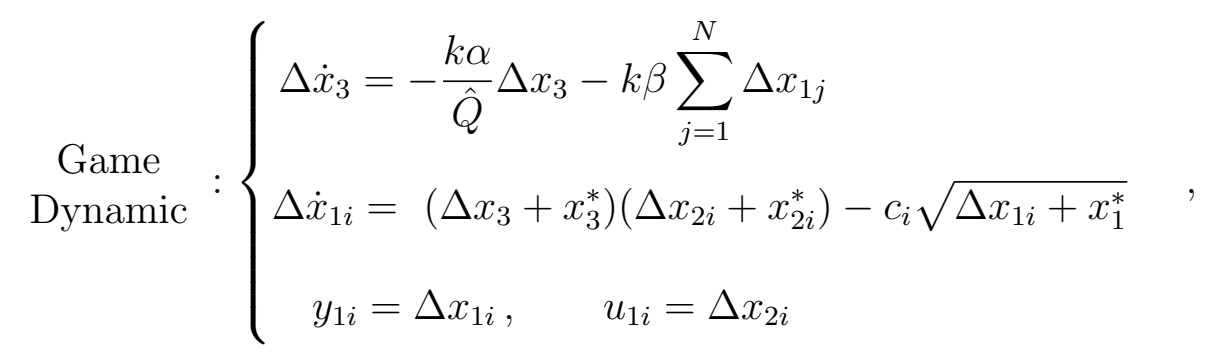

for $i=1, \ldots, N$. In this setup, the Replicator Dynamic has been replaced by the BNN dynamic as the evolutionary dynamic denoted by $\Sigma_{2}$. Also the game dynamic shown by the equation 5.20 is the concatenated system $\Sigma_{1}^{\prime}$ as shown in Figure 5.1 .

The following theorem considers stability characterization of the new system. The theorem below and Theorem 5.3 in the next part are taken from [17, 36] and are slightly modified.

Theorem 5.2. The origin of the system described by equations (5.19) and (5.20) is asymptotically stable.

Proof: Let $\mathcal{V}$ be the candidate Lyapunov function as follows

$$
\mathcal{V}\left(\Delta x_{1}, \Delta x_{2}, \Delta x_{3}\right)=\frac{v_{m}}{r} \sum_{i=1}^{N} \int_{0}^{\left(\Delta x_{3}+x_{3}\right)^{1 / 2} \hat{f}_{i}}[s]_{+} d s+\frac{1}{2} \sum_{i=1}^{N}\left(\Delta \dot{x}_{1 i}\right)^{2}+\frac{m}{2 k \beta}\left(\Delta \dot{x}_{3}\right)^{2}
$$

where $m$ is a positive constant. It is clear that the last two terms on the RHS of the above equation are positive definite. For the first term, consider

$$
z(w)=\int_{0}^{w}[s]_{+} d s
$$

where $[s]_{+}$is the projection defined in equation (2.10). By taking the derivative of $z(w)$ with respect to $w$, we get: $d z / d w=[w]_{+}$, which means that equation 5.22 is nondecreasing for $w>0$ and constant when $w \leqslant 0$. Since $z(w)$ is continuous due to the continuity and integrability of $[s]_{+}$and also the fact that $z(0)=0$, one can conclude 
that (5.22) remains zero for negative $w$. Therefore, the first term on the RHS of equation (5.21) is always non-negative. So the candidate function $\mathcal{V}(\Delta x)$ is positive, moreover, $\mathcal{V}(\Delta x)=0$ if and only if $\Delta x=\left[\Delta x_{1}^{T}, \Delta x_{2}^{T}, \Delta x_{3}\right]^{T}=0$. Thus, $\mathcal{V}(\Delta x)$ is a valid positive definite Lyapunov function. By taking the derivative of $\mathcal{V}$, we obtain

$$
\dot{\mathcal{V}}=\sum_{j=1}^{N} \frac{\partial \mathcal{V}}{\partial \Delta x_{1 j}} \Delta \dot{x}_{1 j}+\sum_{j=1}^{N} \frac{\partial \mathcal{V}}{\partial \Delta x_{2 j}} \Delta \dot{x}_{2 j}+\frac{\partial \mathcal{V}}{\partial \Delta x_{3}} \Delta \dot{x}_{3}
$$

The first term of equation (5.23) is given by

$$
\begin{aligned}
\sum_{j=1}^{N} \frac{\partial \mathcal{V}}{\partial \Delta x_{1 j}} \Delta \dot{x}_{1 j}= & \left(\Delta x_{3}+x_{3}^{*}\right) \sum_{j=1}^{N}\left[\left(\Delta x_{2 j}+x_{2 j}^{*}\right) \sum_{k=1}^{N}\left[\hat{f}_{k}\right]_{+}-\left[\hat{f}_{j}\right]_{+}\right] \Delta \dot{x}_{1 j} \\
& -\frac{1}{2} \sum_{j=1}^{N} c_{j} \frac{\left(\Delta \dot{x}_{1 j}\right)^{2}}{\sqrt{\Delta x_{1 j}+x_{1}^{*}}}-m \Delta \dot{x}_{3} \sum_{j=1}^{N} \Delta \dot{x}_{1 j} \\
= & -\left(\Delta x_{3}+x_{3}^{*}\right) \sum_{j=1}^{N} \Delta \dot{x}_{2 j} \Delta \dot{x}_{1 j}-\frac{1}{2} \sum_{j=1}^{N} c_{j} \frac{\left(\Delta \dot{x}_{1 j}\right)^{2}}{\sqrt{\Delta x_{1 j}+x_{1}^{*}}} \\
& -m \Delta \dot{x}_{3} \sum_{j=1}^{N} \Delta \dot{x}_{1 j} .
\end{aligned}
$$

The second term of equation (5.23) is the following:

$$
\begin{aligned}
\sum_{j=1}^{N} \frac{\partial \mathcal{V}}{\partial \Delta x_{2 j}} \Delta \dot{x}_{2 j} & =\left(\Delta x_{3}+x_{3}^{*}\right) \sum_{k=1}^{N}\left[f_{k}\right]_{+} \sum_{j=1}^{N} \Delta x_{1 j} \Delta \dot{x}_{2 j}+\sum_{j=1}^{N}\left(\Delta x_{3}+x_{3}^{*}\right) \Delta \dot{x}_{2 j} \Delta \dot{x}_{1 j} \\
& =-\frac{v_{m}}{r}\left(\Delta x_{3}+x_{3}^{*}\right) f^{T} \Delta \dot{x}_{2} \sum_{j=1}^{N}\left[\hat{f}_{j}\right]_{+}+\left(\Delta x_{3}+x_{3}^{*}\right) \sum_{j=1}^{N} \Delta \dot{x}_{2 j} \Delta \dot{x}_{1 j}
\end{aligned}
$$

And for the third term we have

$$
\frac{\partial \mathcal{V}}{\partial \Delta x_{3}} \Delta \dot{x}_{3}=\frac{v_{m}}{2 r} \Delta \dot{x}_{3} \sum_{j=1}^{N} \hat{f}_{j}\left[\hat{f}_{j}\right]_{+}+\Delta \dot{x}_{3} \sum_{j=1}^{N}\left(\Delta x_{2 j}+x_{2 j}^{*}\right) \Delta \dot{x}_{1 j}-m \frac{\alpha}{\beta \hat{Q}}\left(\Delta \dot{x}_{3}\right)^{2}
$$


Then, sum of all terms of (5.23) in terms of a quadratic form satisfies

$$
\begin{aligned}
\dot{\mathcal{V}}= & -\left[\begin{array}{ll}
\Delta \dot{x}_{3} & \Delta \dot{x}_{1}^{T}
\end{array}\right]\left[\begin{array}{cc}
\frac{m \alpha}{\beta \hat{Q}} & \frac{1}{2}\left(m \mathbf{1}-\left(\Delta x_{2}+x_{2}^{*}\right)\right)^{T} \\
\frac{1}{2}\left(m \mathbf{1}-\left(\Delta x_{2}+x_{2}^{*}\right)\right) & \frac{1}{2} \operatorname{diag}\left(\frac{c_{i}}{\sqrt{\Delta x_{1 i}+x_{1}^{*}}}\right)
\end{array}\right]\left[\begin{array}{c}
\Delta \dot{x}_{3} \\
\Delta \dot{x}_{1}
\end{array}\right] \\
& -\frac{v_{m}}{r}\left(\Delta x_{3}+x_{3}^{*}\right) f^{T} \Delta \dot{x}_{2} \sum_{j=1}^{N}\left[\hat{f}_{j}\right]_{+}+\frac{v_{m}}{2 r} \Delta \dot{x}_{3} \sum_{j=1}^{N} \hat{f}_{j}\left[\hat{f}_{j}\right]_{+} \\
= & \theta_{5}+\theta_{6}+\theta_{7}
\end{aligned}
$$

where

$$
\begin{aligned}
& \theta_{5}=-\frac{v_{m}}{r}\left(\Delta x_{3}+x_{3}^{*}\right) f^{T} \Delta \dot{x}_{2} \sum_{j=1}^{N}\left[\hat{f}_{j}\right]_{+}, \\
& \theta_{6}=\frac{v_{m}}{2 r} \Delta \dot{x}_{3} \sum_{j=1}^{N} \hat{f}_{j}\left[\hat{f}_{j}\right]_{+}, \\
& \theta_{7}=-\left[\begin{array}{ll}
\Delta \dot{x}_{3} & \Delta \dot{x}_{1}^{T}
\end{array}\right] \mathcal{A}\left[\begin{array}{c}
\Delta \dot{x}_{3} \\
\Delta \dot{x}_{1}
\end{array}\right],
\end{aligned}
$$

and

$$
\mathcal{A}=\left[\begin{array}{cc}
\frac{m \alpha}{\beta \hat{Q}} & \frac{1}{2}\left(m \mathbf{1}-\left(\Delta x_{2}+x_{2}^{*}\right)\right)^{T} \\
\frac{1}{2}\left(m \mathbf{1}-\left(\Delta x_{2}+x_{2}^{*}\right)\right) & \frac{1}{2} \operatorname{diag}\left(\frac{c_{i}}{\sqrt{\Delta x_{1 i}+x_{1}^{*}}}\right)
\end{array}\right]
$$

Due to the positive correlation, $\theta_{5}$ is negative definite but for $\theta_{6}$, the sign depends on $\Delta \dot{x}_{3}$. Also for the matrix $\mathcal{A}$ to be positive definite, we need that $m$ satisfies the inequality below (see Lemma A.1. Appendix A

$$
\frac{2 m \alpha}{\beta \hat{Q}}>\sum_{i=1}^{N} \frac{1}{c_{i}}\left(m-\left(\Delta x_{2 i}+x_{2 i}^{*}\right)\right)^{2} \sqrt{\Delta x_{1 i}+x_{1}^{*}} .
$$


By considering the above inequality to find an appropriate $m$ and using the similar techniques in deriving the D.o.A utilized in section 5.1.1, for the following inequality:

$$
\left|\theta_{6}\right|<\left|\theta_{5}+\theta_{7}\right|
$$

one can approximate the D.o.A for the system. Thus, within this D.o.A,

$$
\theta_{5}+\theta_{6}+\theta_{7}<0
$$

and

$$
\dot{\mathcal{V}}<0
$$

So, by Theorem 3.1, the origin of the system shown in equations (5.19) and (5.20) will be locally asymptotically stable.

\subsubsection{The Smith Dynamic}

Another set of dynamics that satisfies both (PC) and (NS) is the Smith dynamic. Recalling equation (2.25) for the Smith dynamic, we have

$$
\dot{p}_{i}=\sum_{j \in S} p_{j}\left[f_{i}(p)-f_{j}(p)\right]_{+}-p_{i} \sum_{j \in S}\left[f_{j}(p)-f_{i}(p)\right]_{+} .
$$

where by the payoff function shown in equation (4.7) in standard notations, the Smith dynamic takes the following form:

$$
\Delta \dot{x}_{2 i}=\frac{r}{v_{m}} \sum_{j=1}^{N}\left(\Delta x_{2 j}+x_{2 j}^{*}\right)\left[\Delta x_{1 j}-\Delta x_{1 i}\right]_{+}-\frac{r}{v_{m}}\left(\Delta x_{2 i}+x_{2 i}^{*}\right) \sum_{j=1}^{N}\left[\Delta x_{1 i}-\Delta x_{1 j}\right]_{+} .
$$


Now if we write the input-output model of the Smith dynamic as $\Sigma_{2}$ in Figure 5.1, we get:

$$
\underset{\text { Dymith }}{\text { Dynamic }}:\left\{\begin{aligned}
\Delta \dot{x}_{2 i} & =\sum_{j=1}^{N}\left(\Delta x_{2 j}+x_{2 j}^{*}\right)\left[f_{i}-f_{j}\right]_{+}-\left(\Delta x_{2 i}+x_{2 i}^{*}\right) \sum_{j=1}^{N}\left[f_{j}-f_{i}\right]_{+} \\
y_{2 i} & =-\Delta x_{2 i}, \quad u_{2 i}=\Delta x_{1 i}
\end{aligned}\right.
$$

where $i, j=1, \ldots, N$. The following theorem considers the stability of the feedback interconnection of the Game dynamics and the Smith dynamic, shown by equations (5.20) and (5.29), respectively.

Theorem 5.3. The origin of the negative feedback interconnection of two system described by equations (5.20) and (5.29) is asymptotically stable with the following candidate Lyapunov function:

$$
\mathcal{V}=\frac{v_{m}}{r} \sum_{i=1}^{N}\left(\Delta x_{2 i}+x_{2 i}^{*}\right)\left(\sum_{j=1}^{N} \int_{0}^{\left(\Delta x_{3}+x_{3}\right)^{1 / 2}\left(f_{j}-f_{i}\right)}[s]_{+} d s\right)+\frac{1}{2} \sum_{i=1}^{N}\left(\Delta \dot{x}_{1 i}\right)^{2}+\frac{m}{2 k \beta}\left(\Delta \dot{x}_{3}\right)^{2}
$$

The proof of Theorem 5.3 is presented in Appendix A.2.

\subsection{Summary}

In this section, the feedback interconnection of the WDS and the controllers along with the stability analysis were presented. Also the advantage of using the BNN and the Smith dynamics instead of the Replicator Dynamic that guarantees that the feedback interconnected system does not admit non-Nash equilibrium points, was discussed. But there still exists a problem that can affect the equilibrium points and the desired behavior of the system. That is when the system gets disturbed by an external input, i.e., when the input $u$ is not zero. This disturbance could be an extra water consumption or any kind of fluctuations in the input or output of the tanks that can make the system not 
converge to the desirable equilibrium points.

In the next section, we propose an extra subsystem, which highlights the role of the total input flow controller. This will augment controllers of the system and will reject certain kinds of disturbance as well as maintain the desired level of water in all of the tanks. 


\section{Chapter 6}

\section{Disturbance Rejection}

In this part, we assume that the WDS is being disturbed by external inputs. The objective is to reject the disturbance and force the system to converge to the desired equilibrium point while a disturbance is being applied to the system. First in Section 6.1, the model of a subsystem to be added to the system is introduced. Then in Section 6.2, the analysis is provided.

\subsection{Modeling}

In this section, we assume that the input $u$, shown in Figure 3.2 , plays the role of a disturbance to the system. This disturbance could be considered as an extra usage of water, a change in water consumption pattern or type of the demands or a problem in piping system which has to deliver water to the consumers. It could cause a difference between the actual and the desired volume in the tanks, so the main task is to reject the disturbance. In this problem, it is assumed that the disturbance is a step-like (constant) function that is applied to the system for a limited time interval.

One of the simplest and most widely used methods for disturbance rejection in industry is adding an integrator, a PI or a PID controller to the system with a feedback loop to measure the system's error. However, in our system, since the model has the feedback 
interconnection and the states are being analyzed in the error coordinate, we just need to add an integrator to the feedback loop of the gradient controller. So the controller measures the error and makes an appropriate input for the system to force the volume of each tank back to the desired level and maintain it. The new subsystem to be augmented is $\Sigma_{4}$ which is an integrator with gain of $k_{1}>0$. As can be seen from Figures 6.1 and 6.2. there is another feedback loop with the gain of $k_{2}>0$. This feedback loop is based on pole placement to move the pole of the integrator from the origin to the OLHP. In the following discussion, the passivity analysis of the new system is discussed.

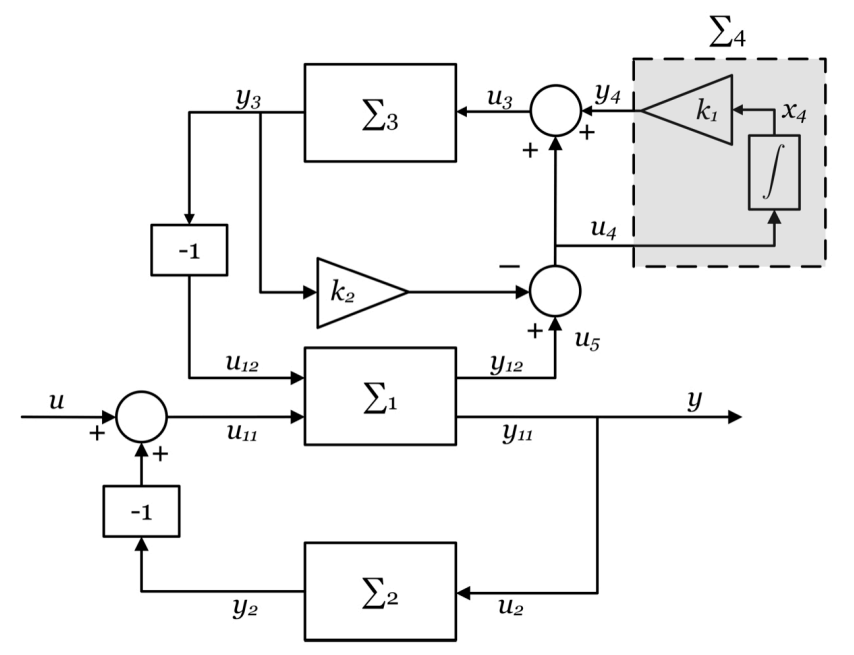

Figure 6.1: Added integrator to the feedback interconnected system.

Consider the upper feedback loop of the system that consists of the gradient controller and the integrator as shown in the following:

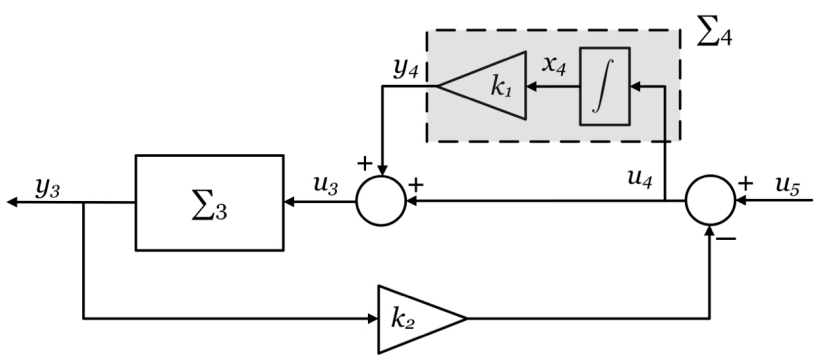

Figure 6.2: Integrator and the gradient controller, $\Sigma_{3}^{\prime}$. 
The corresponding equations for the subsystems represented above are

$$
\begin{aligned}
& \Sigma_{3}:\left\{\begin{array}{c}
\Delta \dot{x}_{3}=-\frac{k \alpha}{\hat{Q}} \Delta x_{3}-k \beta u_{3} \\
y_{3}=-\Delta x_{3}
\end{array}\right. \\
& \Sigma_{4}:\left\{\begin{array}{l}
\dot{x}_{4}=u_{4} \\
y_{4}=k_{1} x_{4}
\end{array}\right.
\end{aligned}
$$

where $x_{4}$ is the state, $u_{4}$ is the input and $y_{4}$ is the output of the integrator. Notice that since this subsystem is placed in a feedback loop and the input $u_{5}=y_{12}=\sum_{j}\left(\Delta x_{1 j}\right)$ is the sum of the states of the plant (see equation (4.26) and Figure 6.1), the input is zero when the interconnected system is at its equilibria. So we can conclude that the steady state of the integrator is also zero, i.e., $x_{4}^{*}=0$. Therefore, $\Delta x_{4}=x_{4}-x_{4}^{*}=x_{4}$ and

$$
\Sigma_{4}:\left\{\begin{array}{l}
\Delta \dot{x}_{4}=u_{4} \\
y_{4}=k_{1} \Delta x_{4}
\end{array}\right.
$$

Now we concatenate these two subsystems, $\Sigma_{3}$ and $\Sigma_{4}$, and call the augmented subsystem $\Sigma_{3}^{\prime}$. Before analyzing the passivity of these subsystems, let us rewrite the equations to get a second order LTI SISO system from input $u_{5}$ to output $y_{3}$. From Figure 6.2, clearly we get

$$
\begin{aligned}
& u_{4}=u_{5}-k_{2} y_{3}=u_{5}+k_{2} \Delta x_{3}, \\
& u_{3}=u_{4}+y_{4}=u_{5}+k_{2} \Delta x_{3}+k_{1} \Delta x_{4} .
\end{aligned}
$$

Therefore, the differential equations become

$$
\begin{aligned}
& \Delta \dot{x}_{3}=-\left(\frac{k \alpha}{\hat{Q}}+k \beta k_{2}\right) \Delta x_{3}-k \beta k_{1} \Delta x_{4}-k \beta u_{5}, \\
& \Delta \dot{x}_{4}=k_{2} \Delta x_{3}+u_{5}
\end{aligned}
$$


and $\Sigma_{3}^{\prime}$ will be of form

$$
\begin{aligned}
\Delta \dot{x}_{3}^{\prime} & =\left[\begin{array}{cc}
-\left(\frac{k \alpha}{\hat{Q}}+k \beta k_{2}\right) & -k \beta k_{1} \\
k_{2} & 0
\end{array}\right] \Delta x_{3}^{\prime}+\left[\begin{array}{c}
-k \beta \\
1
\end{array}\right] u_{5}, \\
y_{3} & =\left[\begin{array}{ll}
-1 & 0
\end{array}\right] \Delta x_{3}^{\prime},
\end{aligned}
$$

where $\Delta x_{3}^{\prime}=\left[\Delta x_{3}, \Delta x_{4}\right]^{T}$.

In the next section, stability of the augmented system is characterized.

\subsection{Analysis}

The theorem below considers the passivity characterization of $\Sigma_{3}^{\prime}$ :

Theorem 6.1. The linear time-invariant system (6.4) and 6.5) is passive from input $u_{5}$ to output $y_{3}$ if $k_{1} \leqslant \frac{k \alpha}{\hat{Q}}+k \beta k_{2}$. Furthermore, if $k_{1}<\frac{k \alpha}{\hat{Q}}+k \beta k_{2}$, then the system is strictly passive.

Proof: First, we calculate the transfer function of $\Sigma_{3}^{\prime}$ by

$$
G(s)=C(s I-A)^{-1} B
$$

where

$$
A=\left[\begin{array}{cc}
-\left(\frac{k \alpha}{\hat{Q}}+k \beta k_{2}\right) & -k \beta k_{1} \\
k_{2} & 0
\end{array}\right], \quad B=\left[\begin{array}{c}
-k \beta \\
1
\end{array}\right] \quad \text { and } \quad C=\left[\begin{array}{cc}
-1 & 0
\end{array}\right]
$$

So we get

$$
G(s)=\frac{k \beta\left(s+k_{1}\right)}{s^{2}+s\left(\frac{k \alpha}{\hat{Q}}+k \beta k_{2}\right)+k \beta k_{1} k_{2}} .
$$

Clearly, since all of the parameters are positive, poles of $\Sigma_{3}^{\prime}$ are in the OLHP. By letting $s=j \omega$ we get 


$$
\begin{aligned}
G(j \omega) & =\frac{k \beta\left(j \omega+k_{1}\right)}{(j \omega)^{2}+j \omega\left(\frac{k \alpha}{\hat{Q}}+k \beta k_{2}\right)+k \beta k_{1} k_{2}} \\
& =\frac{k \beta k_{1}\left(k \beta k_{1} k_{2}-\omega^{2}\right)+k \beta \omega^{2}\left(\frac{k \alpha}{\hat{Q}}+k \beta k_{2}\right)}{\left(k \beta k_{1} k_{2}-\omega^{2}\right)^{2}+\omega^{2}\left(\frac{k \alpha}{\hat{Q}}+k \beta k_{2}\right)^{2}}-j \frac{k \beta \omega^{3}+\frac{k^{2} \beta \alpha k_{1}}{\hat{Q}} \omega}{\left(k \beta k_{1} k_{2}-\omega^{2}\right)^{2}+\omega^{2}\left(\frac{k \alpha}{\hat{Q}}+k \beta k_{2}\right)^{2}} .
\end{aligned}
$$

Now to get $\operatorname{Re}[G(j \omega)]>0$ we should have

$$
k \beta k_{1}\left(k \beta k_{1} k_{2}-\omega^{2}\right)+k \beta \omega^{2}\left(\frac{k \alpha}{\hat{Q}}+k \beta k_{2}\right)>0 .
$$

By rewriting the inequality we get

$$
k^{2} \beta^{2} k_{1}^{2} k_{2}+k \beta \omega^{2}\left(\frac{k \alpha}{\hat{Q}}+k \beta k_{2}-k_{1}\right)>0 .
$$

So by considering

$$
k_{1}<\frac{k \alpha}{\hat{Q}}+k \beta k_{2}
$$

the real part of $G(j \omega)$ is positive definite. Thus, by Definition 3.2, the transfer function of $\Sigma_{3}^{\prime}$ is strictly positive real and by Lemma $3.2, \Sigma_{3}^{\prime}$ is strictly passive.

We just proved that the new subsystem, $\Sigma_{3}^{\prime}$, under condition 6.6 is strictly passive. So by the same method discussed in Chapter 5, by Theorem 3.3, we can say that all components of the feedback interconnection system $\Sigma_{1}, \Sigma_{2}$ and $\Sigma_{3}$, shown in Figure 6.1. are passive and hence the system is strictly passive within a D.o.A. Therefore, by lemma 3.1, the equilibrium point will be locally asymptotically stable for zero inputs.

\subsection{Summary}

This section presented an augmented subsystem to reject external disturbances to the system. This subsystem, which is an integrator, can make the error zero in converging 
to the desired $N E$ when a step-like disturbance is applied to the system. The stability analysis followed the same procedure discussed in the previous chapters by characterizing the passivity of the interconnected system and deriving a condition under which the system remains strictly passive.

In Chapter 5, it is illustrated that the system is asymptotically stable but not globally asymptotically stable. So we have to derive a D.o.A in which the system remains stable. In the next section, we show that by a modification on the system, we can achieve global stability. 


\section{Chapter 7}

\section{Global Convergence; Generalization}

This chapter presents a modification to the feedback interconnected system to achieve global convergence to the desirable NE's. Also an extension to the WDS system as a generalization to a certain class of population games is presented. In Section 7.1, the modified systems along with the global convergence analysis are provided. Next, in Section 7.2, a general class of population games of which the water distribution system is an example as well as a discussion about their stability and convergence properties is introduced.

\subsection{Globally Asymptotic Stability}

In this part, in order to get a globally asymptotically stable feedback interconnection of Figure 4.3, described by equations (4.26), 4.27) and (4.30), we reduce the degree of freedom in our system. This can be done by considering the total input flow of the system as a constant parameter $Q^{*}$ that can be externally tuned based on the demands and the desired level of water in the tanks. This parameter can be calculated by solving equations 4.18 - 4.20) for different values of $v^{*}$. Therefore, the system dynamics take 
the form of

$$
\begin{gathered}
\underset{\text { Game }}{\text { Dynamic }}:\left\{\begin{array}{c}
\Delta \dot{x}_{1 i}=Q^{*}\left(\Delta x_{2 i}+x_{2 i}^{*}\right)-c_{i} \sqrt{\Delta x_{1 i}+x_{1}^{*}} \\
y_{1 i}=\Delta x_{1 i}, \quad u_{1 i}=\Delta x_{2 i}
\end{array}\right. \\
\underset{\text { Dynamic }}{\text { Dynamic }}:\left\{\begin{array}{c}
\Delta \dot{x}_{2 i}=\left[\hat{f}_{i}\right]_{+}-\left(\Delta x_{2 i}+x_{2 i}^{*}\right) \sum_{j=1}^{N}\left[\hat{f}_{j}\right]_{+} \\
y_{2 i}=-\Delta x_{2 i}, \quad u_{2 i}=\Delta x_{1 i}
\end{array}\right.
\end{gathered}
$$

or

$$
\underset{\text { Dynith }}{\text { Dynamic }}:\left\{\begin{array}{rl}
\Delta \dot{x}_{2 i} & =\sum_{j=1}^{N}\left(\Delta x_{2 j}+x_{2 j}^{*}\right)\left[f_{i}-f_{j}\right]_{+}-\left(\Delta x_{2 i}+x_{2 i}^{*}\right) \sum_{j=1}^{N}\left[f_{j}-f_{i}\right]_{+} \\
y_{2 i} & =-\Delta x_{2 i}, \quad u_{2 i}=\Delta x_{1 i}
\end{array} .\right.
$$

where $f_{i}$ and $\hat{f}_{i}$ are defined in equations 4.7 and (5.18), respectively. These dynamical systems could be placed in the negative feedback interconnection, shown in the following figure

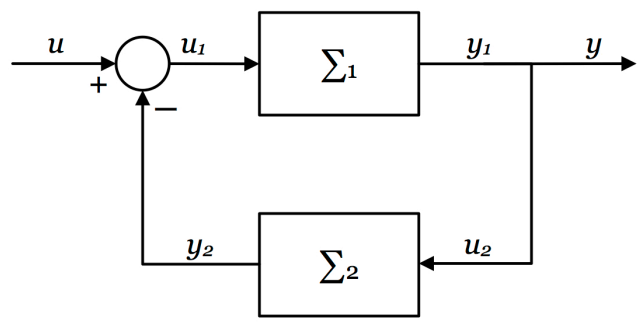

Figure 7.1: Negative feedback interconnection of the game and the evolutionary dynamic.

where $\Sigma_{1}$ is the game dynamic in form of equation (7.1) and $\Sigma_{2}$ could be either the BNN dynamic, equation (7.2), or the Smith dynamic, equation (7.3), as the evolutionary dynamic. In this configuration, we are looking for global convergence to the desirable $N E$ 's. Since the Replicator Dynamic, due to the (NS) property, admits non-Nash equilibrium points, we do not include pairwise proportional imitative dynamics in this set of evolutionary dynamics. Thus, we only use the BNN and the Smith dynamics to assure 
that the global convergence to the desired NE's is achievable. To show the passivity of the game dynamic and the evolutionary dynamic that are introduced by the equations (7.1), (7.2) and (7.3), we utilize a new approach adapted from [17, 18]. We reformulate our systems of the form (3.1) by

$$
\begin{aligned}
& \dot{u}=u_{e}, \\
& \dot{x}=f(x, u), \\
& y_{e}=\dot{y}=\nabla_{x} h(x, u) \dot{x}+\nabla_{u} h(x, u) \dot{u},
\end{aligned}
$$

and call them 'Extended Systems'. These systems have input and output of $u_{e}$ and $y_{e}$ that are the time derivatives of the original input and the output of the main system, respectively, and also a new state vector of $x_{e}=(x, u)$. Similarly, we can extend the definition of passivity for this system by finding a storage function $\mathcal{V}\left(x_{e}\right)$ such that the following holds:

$$
\dot{\mathcal{V}} \leqslant u_{e}^{T} y_{e}=\dot{u}^{T} \dot{y}
$$

This new configuration avails us to analyze the passivity and stability of the systems, introduced in equations $(7.2)$ and $(7.3)$, by using the existing Lyapunov functions found in [21, 35, 36] as storage functions. By using this approach, we can construct the extended evolutionary dynamic and extended WDS as the extended game dynamic defined by

$$
\begin{aligned}
\underset{\text { Extended }}{\text { Game Dynamic }}: & \left\{\begin{array}{l}
\Delta \dot{x}_{1 i}=Q^{*}\left(\Delta x_{2 i}+x_{2 i}^{*}\right)-c_{i} \sqrt{\Delta x_{1 i}+x_{1}^{*}} \\
\dot{u}_{1 i}=\Delta \dot{x}_{2 i}=u_{e_{1 i}} \\
y_{e_{1 i}}=\dot{y}_{1 i}=\Delta \dot{x}_{1 i}
\end{array}\right. \\
\underset{\text { BNN Dynamic }}{\text { Extended }} & :\left\{\begin{array}{l}
\Delta \dot{x}_{2 i}=\left[\hat{f}_{i}\right]_{+}-\left(\Delta x_{2 i}+x_{2 i}^{*}\right) \sum_{j=1}^{N}\left[\hat{f}_{j}\right]_{+} \\
\dot{u}_{2 i}=\Delta \dot{x}_{1 i}=u_{e_{2 i}} \\
y_{e_{2 i}}=\dot{y}_{2 i}=-\Delta \dot{x}_{2 i}
\end{array}\right.
\end{aligned}
$$


or

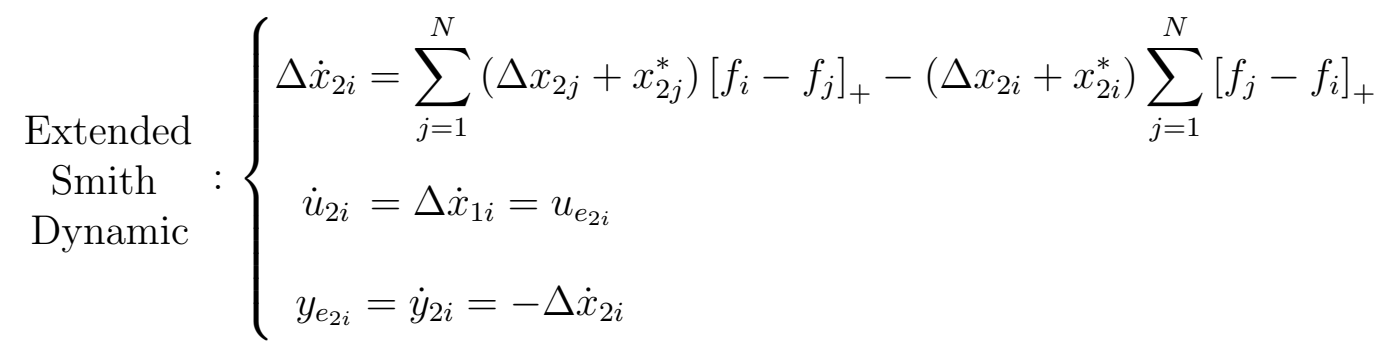

Theorems below, which are adapted from [17, 36] and are slightly modified, capture the passivity analysis of the the systems introduced by equations (7.6), (7.7) and (7.8).

Theorem 7.1. The extended BNN evolutionary dynamic, shown in equation (7.7), is a strictly passive mapping from the payoff trajectories to the strategy trajectories with the following positive definite storage function:

$$
\mathcal{V}_{1}\left(\Delta x_{1}, \Delta x_{2}\right)=\frac{v_{m}}{r} \sum_{j=1}^{N} \int_{0}^{\hat{f}_{j}}[s]_{+} d s
$$

Proof: By substituting $\hat{f}_{j}$, shown in (5.18), the storage function takes the form of

$$
\mathcal{V}_{1}\left(\Delta x_{1}, \Delta x_{2}\right)=\frac{v_{m}}{r} \sum_{j=1}^{N} \int_{0}^{\frac{r}{v_{m}}\left(\sum_{k=1}^{N}\left(\Delta x_{2 k}+x_{2 k}^{*}\right) \Delta x_{1 k}-\Delta x_{1 j}\right)}[s]_{+} d s
$$

By taking the derivative of the storage function along the trajectories, we get

$$
\begin{aligned}
\dot{\mathcal{V}}_{1} & =\sum_{j=1}^{N} \frac{\partial \mathcal{V}_{1}}{\partial \Delta x_{1 j}} \Delta \dot{x}_{1 j}+\sum_{j=1}^{N} \frac{\partial \mathcal{V}_{1}}{\partial \Delta x_{2 j}} \Delta \dot{x}_{2 j} \\
& =\sum_{j=1}^{N}\left[\left(\Delta x_{2 j}+x_{2 j}^{*}\right) \sum_{k=1}^{N}\left[\hat{f}_{k}\right]_{+}-\left[\hat{f}_{j}\right]_{+}\right] \Delta \dot{x}_{1 j}+\sum_{k=1}^{N}\left[\hat{f}_{k}\right]_{+} \sum_{j=1}^{N} \Delta x_{1 j} \Delta \dot{x}_{2 j} .
\end{aligned}
$$

We use equation $(7.2)$ for the first term of the above equation, so the derivative of $\mathcal{V}_{1}$ will be of form

$$
\dot{\mathcal{V}}_{1}=-\sum_{j=1}^{N} \Delta \dot{x}_{2 j} \Delta \dot{x}_{1 j}-\frac{v_{m}}{r} f^{T} \Delta \dot{x}_{2} \sum_{k=1}^{N}\left[\hat{f}_{k}\right]_{+}=-\Delta \dot{x}_{2}^{T} \Delta \dot{x}_{1}-\frac{v_{m}}{r} f^{T} \Delta \dot{x}_{2} \sum_{k=1}^{N}\left[\hat{f}_{k}\right]_{+}
$$


where due to non-negativity of $\left[f_{j}\right]_{+}$and the positive correlation property of BNN dynamics, the second term on the RHS of the above equation is negative definite. So

$$
\dot{\mathcal{V}}_{1}<u_{e_{2}}^{T} y_{e_{2}}
$$

which proves that the BNN dynamic is strictly passive.

Theorem 7.2. The extended Smith evolutionary dynamic, shown in equation (7.8), is a strictly passive mapping from the payoff trajectories to the strategy trajectories with the following positive definite storage function:

$$
\mathcal{V}_{2}\left(\Delta x_{1}, \Delta x_{2}\right)=\frac{v_{m}}{r} \sum_{i=1}^{N}\left(\Delta x_{2 i}+x_{2 i}^{*}\right) \sum_{j=1}^{N} \int_{0}^{f_{j}-f_{i}}[s]_{+} d s
$$

Proof: By taking the derivative of the storage function along the trajectories, we get

$$
\dot{\mathcal{V}}_{2}=\sum_{j=1}^{N} \frac{\partial \mathcal{V}_{2}}{\partial \Delta x_{1 j}} \Delta \dot{x}_{1 j}+\sum_{j=1}^{N} \frac{\partial \mathcal{V}_{2}}{\partial \Delta x_{2 j}} \Delta \dot{x}_{2 j}
$$

The first term of equation 7.12 is given by

$$
\begin{aligned}
\sum_{j=1}^{N} \frac{\partial \mathcal{V}_{2}}{\partial \Delta x_{1 j}} \Delta \dot{x}_{1 j} & =\sum_{j=1}^{N} \Delta \dot{x}_{1 j}\left(\left(\Delta x_{2 j}+x_{2 j}^{*}\right) \sum_{i=1}^{N}\left[f_{i}-f_{j}\right]_{+}-\sum_{i=1}^{N}\left(\Delta x_{2 i}+x_{2 i}^{*}\right)\left[f_{j}-f_{i}\right]_{+}\right) \\
& =-\sum_{j=1}^{N} \Delta \dot{x}_{2 j} \Delta \dot{x}_{1 j}=-\Delta \dot{x}_{2}^{T} \Delta \dot{x}_{1}=u_{e_{2}}^{T} y_{e_{2}}
\end{aligned}
$$

and the second term of equation $(7.12)$ is the following:

$$
\sum_{j=1}^{N} \frac{\partial \mathcal{V}_{2}}{\partial \Delta x_{2 j}} \Delta \dot{x}_{2 j}=\frac{v_{m}}{r} \sum_{j=1}^{N} \Delta \dot{x}_{2 j} \sum_{k=1}^{N} \int_{0}^{f_{k}-f_{j}}[s]_{+} d s
$$


where $\Delta \dot{x}_{2 j}$ is shown in equation 7.8 and $\int_{0}^{f_{k}-f_{j}}[s]_{+} d s$ is a non-decreasing function. By expanding the equations, the RHS of equation (7.13) will take the form of

$$
\begin{aligned}
& \sum_{j=1}^{N}\left(\sum_{i=1}^{N}\left(\Delta x_{2 i}+x_{2 i}^{*}\right) \rho_{i j}-\left(\Delta x_{2 j}+x_{2 j}^{*}\right) \sum_{i=1}^{N} \rho_{j i}\right) \sum_{k=1}^{N} \int_{0}^{f_{k}-f_{j}}[s]_{+} d s \\
= & \sum_{j=1}^{N} \sum_{i=1}^{N}\left(\Delta x_{2 i}+x_{2 i}^{*}\right) \rho_{i j} \sum_{k=1}^{N} \int_{0}^{f_{k}-f_{j}}[s]_{+} d s-\sum_{j=1}^{N} \sum_{i=1}^{N}\left(\Delta x_{2 j}+x_{2 j}^{*}\right) \rho_{j i} \sum_{k=1}^{N} \int_{0}^{f_{k}-f_{j}}[s]_{+} d s .
\end{aligned}
$$

Now by switching $j$ and $i$ in the second term of the above equation, we get

$$
\begin{aligned}
& \sum_{j=1}^{N} \sum_{i=1}^{N}\left(\Delta x_{2 i}+x_{2 i}^{*}\right) \rho_{i j} \sum_{k=1}^{N} \int_{0}^{f_{k}-f_{j}}[s]_{+} d s-\sum_{i=1}^{N} \sum_{j=1}^{N}\left(\Delta x_{2 i}+x_{2 i}^{*}\right) \rho_{i j} \sum_{k=1}^{N} \int_{0}^{f_{k}-f_{i}}[s]_{+} d s \\
= & \sum_{j=1}^{N} \sum_{i=1}^{N}\left(\Delta x_{2 i}+x_{2 i}^{*}\right)\left[f_{j}-f_{i}\right]_{+} \sum_{k=1}^{N}\left(\int_{0}^{f_{k}-f_{j}}[s]_{+} d s-\int_{0}^{f_{k}-f_{i}}[s]_{+} d s\right) .
\end{aligned}
$$

To evaluate the above equation, first observe that $\left[f_{j}-f_{i}\right]_{+}=0$ if $f_{j} \leqslant f_{i}$. But if $f_{j}>f_{i}$, then $\left[f_{j}-f_{i}\right]_{+}>0$ and $f_{k}-f_{j}<f_{k}-f_{i}$; since the integral in equations 7.13 and (7.14) is non-decreasing, it follows that

$$
\int_{0}^{f_{k}-f_{j}}[s]_{+} d s-\int_{0}^{f_{k}-f_{i}}[s]_{+} d s \leqslant 0 .
$$

Also equation 7.14 is zero if and only if $\left[\Delta x_{1}^{T}, \Delta x_{2}^{T}\right]^{T}=0$. Therefore, equation (7.13) is negative definite. Hence, summation of the two terms in equation (7.12) will satisfy

$$
\dot{\mathcal{V}}_{2}<u_{e_{2}}^{T} y_{e_{2}}
$$

which proves that the Smith dynamic is strictly passive.

Now, the theorem below shows the passivity of the extended water distribution system as the extended game. Thus, we can connect the extended game in a negative feedback interconnection to the extended evolutionary dynamic and obtain a strictly passive 
interconnection. Therefore, the theorems presented in Chapter 3 could be utilized to guarantee the convergence of the system to the desirable equilibrium points.

Theorem 7.3. The extended water distribution system as the extended population game dynamic, shown in equation (7.6), is a strictly passive mapping from the strategy trajectories to the payoff trajectories.

Proof: Consider the following positive definite storage function:

$$
\mathcal{V}_{3}=\frac{1}{2 Q^{*}} \sum_{j=1}^{N}\left(\Delta \dot{x}_{1 j}\right)^{2}
$$

By taking the derivative along the trajectories of the system, we obtain

$$
\begin{aligned}
\dot{\mathcal{V}}_{3} & =\sum_{j=1}^{N} \frac{\partial \mathcal{V}_{3}}{\partial \Delta x_{1 j}} \Delta \dot{x}_{1 j}+\sum_{j=1}^{N} \frac{\partial \mathcal{V}_{3}}{\partial \Delta x_{2 j}} \Delta \dot{x}_{2 j} \\
& =-\frac{1}{2 Q^{*}} \sum_{j=1}^{N} c_{j} \frac{\left(\Delta \dot{x}_{1 j}\right)^{2}}{\sqrt{\Delta x_{1 j}+x_{1}^{*}}}+\frac{1}{Q^{*}} \sum_{j=1}^{N} Q^{*} \Delta \dot{x}_{2 j} \Delta \dot{x}_{1 j}
\end{aligned}
$$

It is clear that the first term is strictly negative, so

$$
\begin{aligned}
\dot{\mathcal{V}}_{3} & =u_{e_{1}}^{T} y_{e_{1}}-\frac{1}{2 Q^{*}} \sum_{j=1}^{N} c_{j} \frac{\left(\Delta \dot{x}_{1 j}\right)^{2}}{\sqrt{\Delta x_{1 j}+x_{1}^{*}}} \\
& <u_{e_{1}}^{T} y_{e_{1}}
\end{aligned}
$$

which shows the extended game dynamic is strictly passive.

Now by considering a new storage function of the form

$$
\mathcal{V}_{\text {new }}=\mathcal{V}_{1}+\mathcal{V}_{3}
$$

or

$$
\mathcal{V}_{\text {new }}=\mathcal{V}_{2}+\mathcal{V}_{3}
$$


where $\mathcal{V}_{1}, \mathcal{V}_{2}$ and $\mathcal{V}_{3}$ are defined in equations (7.9), (7.11) and (7.17), respectively, by Theorem 3.2 , we can say that the negative feedback interconnection of the extended game dynamic and the extended evolutionary dynamic is strictly passive. Also since the storage function is radially unbounded, by Lemma 3.1, the equilibrium point is globally asymptotically stable for zero input $(u=0)$.

In the next section, this result is extended for a generalized class of extended dynamically modified population games.

\subsection{Affine Games}

In this section, a general class of dynamically modified population games that includes the WDS is presented. We show that this generalized class of games admits the property of strict passivity and hence, by Theorem 3.2 , its negative feedback interconnection with a set of strictly passive evolutionary dynamic, shown in Figure 7.1, converges to the Nash equilibrium points of the game, which is the desired asymptotically stable equilibrium point of the feedback interconnection system.

Consider a dynamically modified population game, presented in Chapter 4 and described by equation 4.1), in the form of

$$
\dot{x}=F(x, p),
$$

that is being controlled in a negative feedback interconnection, shown in Figure 7.1, by a set of strictly passive evolutionary dynamics, given by

$$
\dot{p}=V(p, f)
$$

where $V(p, f)$ is introduced in (2.8). In this configuration, $x \in \mathbb{R}^{N}$ is the state vector of the game and $p \in \Delta \subset \mathbb{R}^{N}$ is the population state. Since in the setup of dynamically modified game, payoff functions are defined with respect to the game state vector (i.e., 
$f=f(x)$ ), one can reconsider the notation of the evolutionary dynamic as follows

$$
\dot{p}=V(p, f(x))=V(p, x) .
$$

If we model the modified population game and the evolutionary dynamic as the extended input-output systems of form 7.4 , we arrive at

$$
\underset{\text { Extended }}{\text { Modified Game }}:\left\{\begin{array}{l}
\dot{x}=F(x, p) \\
u_{1}=\dot{p}, \quad y_{1}=\dot{x}
\end{array}\right.
$$

and

$$
\begin{gathered}
\text { Extended } \\
\text { Evolutionary Dynamic }
\end{gathered}:\left\{\begin{array}{l}
\dot{p}=V(p, x) \\
u_{2}=\dot{x}, \quad y_{2}=-\dot{p}
\end{array} .\right.
$$

Now we can utilize the configuration of Figure 4.1 and model the connection with a negative feedback interconnection of Figure 7.1 where the extended game and the extended evolutionary dynamic are denoted $\Sigma_{1}$ and $\Sigma_{2}$, respectively. The theorem below sums up the stability analysis of the feedback interconnection mentioned above.

Theorem 7.4. Suppose that a dynamically modified population game in the form of an extended input-output dynamical system is described by equation (7.24) where $F(x, p)$ : $\mathbb{R}^{N} \times \Delta \rightarrow \mathbb{R}^{N}$ is Lipschitz continuous. The negative feedback interconnection of the modified population game with a set of strictly passive evolutionary dynamics described by equation (7.25), shown in Figure 7.1, is strictly passive if

- $F$ is strictly decreasing in $x$, and

- F is linear, affine and strictly increasing in $p$.

Furthermore, for zero input of the interconnection, the origin of the feedback system is asymptotically stable. 
Proof: The first condition of being strictly decreasing means that $\nabla_{x} F<0$ and the second condition says that there exists a symmetric positive definite matrix $\mathcal{M}$, which is independent of $x$, such that $\nabla_{p} F=\mathcal{M}>0$. Thus, we can represent this type of game in the following form:

$$
F(x, p)=\mathcal{M} p+g(x)
$$

where $\nabla_{x} F=\nabla_{x} g<0$. Therefore, if we consider the following storage function for the population game:

$$
L(x, p)=\frac{1}{2} \dot{x}^{T} \mathcal{M}^{-1} \dot{x}=F(x, p)^{T} \mathcal{M}^{-1}(\mathcal{M} p+g(x))
$$

by taking the derivative along the trajectories we obtain

$$
\begin{aligned}
\dot{L} & =\nabla_{x} L(x, p) \dot{x}+\nabla_{p} L(x, p) \dot{p} \\
& =\dot{x}^{T} \mathcal{M}^{-1} \nabla_{x} F \dot{x}+\dot{x}^{T} \mathcal{M}^{-1} \nabla_{p} F \dot{p} \\
& =\dot{x}^{T} \mathcal{M}^{-1} \nabla_{x} g \dot{x}+\dot{x}^{T} \mathcal{M}^{-1} \mathcal{M} \dot{p} \\
& <\dot{x}^{T} \dot{p}=u_{1}^{T} y_{1} .
\end{aligned}
$$

The last inequality is due to the facts that $\nabla_{x} g<0$ and $\mathcal{M}^{-1}>0$. This proves that the dynamically modified game is strictly passive. So if we consider a set of strictly passive evolutionary dynamic of form 27.25 such as the BNN and Smith dynamics, shown in equation (7.7) or 7.8 that are proved to be strictly passive by Theorem 7.1 and Theorem 7.2 , we can conclude by Theorem 3.2 that the negative feedback interconnection of this type of modified games and the corresponding evolutionary dynamic is strictly passive and for zero inputs, the origin of the system is asymptotically stable. Moreover, if $g(x)$ is radially unbounded in $x$, then the storage function $L$ will be radially unbounded and the origin, by Lemma 3.1, will be globally asymptotically stable.

As we showed in the theorem above, any type of population game that could be 
represented in forms of 7.24 and $(7.26)$ is strictly passive and it is guaranteed that its solutions in a feedback interconnection with a set of strictly passive evolutionary dynamic globally converge to the Nash equilibrium of the game.

To sum up this part, consider again the WDS as an example of the population game with dynamic dependencies in standard notation. In this example, the game is presented as an extended input output model in equation (7.24), where

$$
F\left(\Delta x_{1}, \Delta x_{2}\right)=Q^{*}\left(\Delta x_{2 i}+x_{2 i}^{*}\right)-c_{i} \sqrt{\Delta x_{1 i}+x_{1}^{*}}
$$

It can be shown that

$$
\mathcal{M}=\nabla_{\Delta x_{2}} F=Q^{*} I_{N}>0
$$

where $I_{N}$ is the $N \times N$ identity matrix. Also it is immediate that

$$
\nabla_{\Delta x_{1}} F=\left[\begin{array}{ccc}
\nabla_{\Delta x_{11}} F & \ldots & 0 \\
\vdots & \ddots & \vdots \\
0 & \ldots & \nabla_{\Delta x_{1 N}} F
\end{array}\right]<0
$$

since $\nabla_{\Delta x_{1 j}} F<0$ for all $j=1, \ldots, N$ in the corresponding domain $\mathcal{X}$. Therefore, as it is shown in Theorem 7.3 , we can formulate the WDS equations in the form of equation (7.26) and show that it is strictly passive. Thus, it converges to the desired equilibrium points in connection with a strictly passive evolutionary dynamic.

\subsection{Summary}

We presented an extended version of the game dynamic and the evolutionary dynamic to show the passivity and global stability of their negative feedback interconnection. We also introduced a general class of games including the WDS and presented the analysis that confirmed this class of games in connection with the BNN or Smith evolutionary 
dynamics would converge to the $N E$ 's of the game.

In the next chapter, simulation results of the WDS with different classes of evolutionary dynamics are provided. 


\section{Part III}

\section{Simulation and Conclusion}




\section{Chapter 8}

\section{Simulation Results}

In this chapter, we present simulation results of the systems with different configurations discussed in Chapters 4-6. We begin with the Replicator Dynamic in Section 8.1 and show the results. In addition, we discuss the results of the BNN and Smith dynamics for the same parameters of the system. Then in Section 8.2, we present the problem of converging to non-Nash equilibrium points that could occur by using the Replicator Dynamic as the evolutionary dynamic. As a solution, we utilize the BNN and Smith dynamics to solve this problem. In Section 8.3, we discuss the reason why we employed a controller for the total input flow. Also some results about the system disturbance rejection are provided.

\subsection{The Replicator, BNN and Smith Dynamics}

We have used the simple model of the $N$-tank system where the output flows are under the atmospheric pressure. The number of tanks in this simulation is three and the parameters of the system are summarized in Table 8.1. In this section, we show the simulation results of the tank system in connection with the Replicator, BNN and Smith dynamics and the total input flow controller, illustrated in Figure 3.2. In these examples, initial conditions and the parameters remain the same. 


\begin{tabular}{|c|c||c|c|}
\hline Parameter & Value & Parameter & Value \\
\hline$v_{m}$ & $5000 \mathrm{~m}^{3}$ & $C_{1}$ & 1 \\
\hline$r$ & 1 & $C_{2}$ & 1 \\
\hline$S_{1}$ & $1024 \mathrm{~m}^{2}$ & $C_{3}$ & 1 \\
\hline$S_{2}$ & $729 \mathrm{~m}^{2}$ & $c_{1}$ & 0.0555 \\
\hline$S_{3}$ & $441 \mathrm{~m}^{2}$ & $c_{2}$ & 0.0483 \\
\hline$A_{1}$ & $0.3973 \mathrm{~m}^{2}$ & $c_{3}$ & 0.0622 \\
\hline$A_{2}$ & $0.2919 \mathrm{~m}^{2}$ & $g$ & $9.8 \mathrm{~m} / \mathrm{s}^{2}$ \\
\hline$A_{3}$ & $0.2919 \mathrm{~m}^{2}$ & $N$ & 3 \\
\hline \hline Parameter & Value & Parameter & Value \\
\hline$\alpha$ & 4.1350 & $k$ & 0.1 \\
\hline$\beta$ & 0.01 & $\hat{Q}$ & $4 \mathrm{~m}^{3} / \mathrm{s}$ \\
\hline$\gamma$ & 0.2 & & \\
\hline
\end{tabular}

Table 8.1: Tank system and total input flow controller parameters.

\begin{tabular}{|c|c|}
\hline Variable & Value \\
\hline$v(0)$ & {$[30,30,30]^{T} \mathrm{~m}^{3}$} \\
\hline$p(0)$ & {$[0.1,0.2,0.7]^{T}$} \\
\hline$Q(0)$ & $0.1 \mathrm{~m}^{3} / \mathrm{s}$ \\
\hline
\end{tabular}

Table 8.2: System initial condition 1.

Considering equations (4.23) and (4.24), we have $v^{*}=80, p^{*}=[0.3344,0.2912,0.3744]^{T}$ and $Q^{*}=1.4849$ as the desired equilibrium points of the system. In the next figures, we demonstrate the simulation results for the tank system in connection with three different sets of evolutionary dynamic. The first plots are for the Replicator Dynamic in which the system, for initial conditions in the corresponding D.o.A (see Section 5.1), converges 
to the desired equilibrium point:
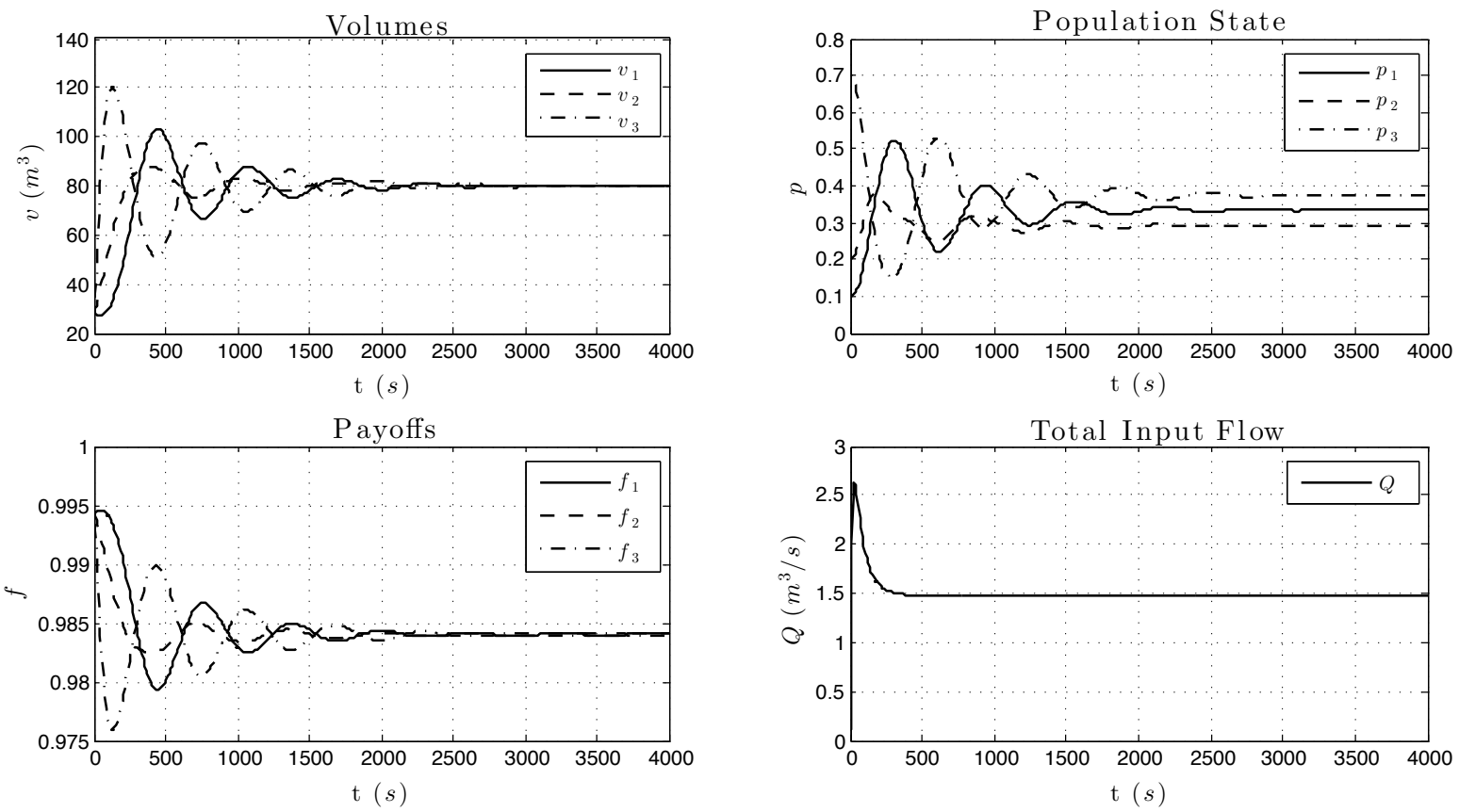

Figure 8.1: Tank system and the Replicator Dynamic, with initial condition 1.

where the Replicator Dynamic has driven the system toward the $N E$; The final volume of the tanks are the same and payoffs are the maximum available to the agents playing this game. Next, we present the results of the BNN dynamic:
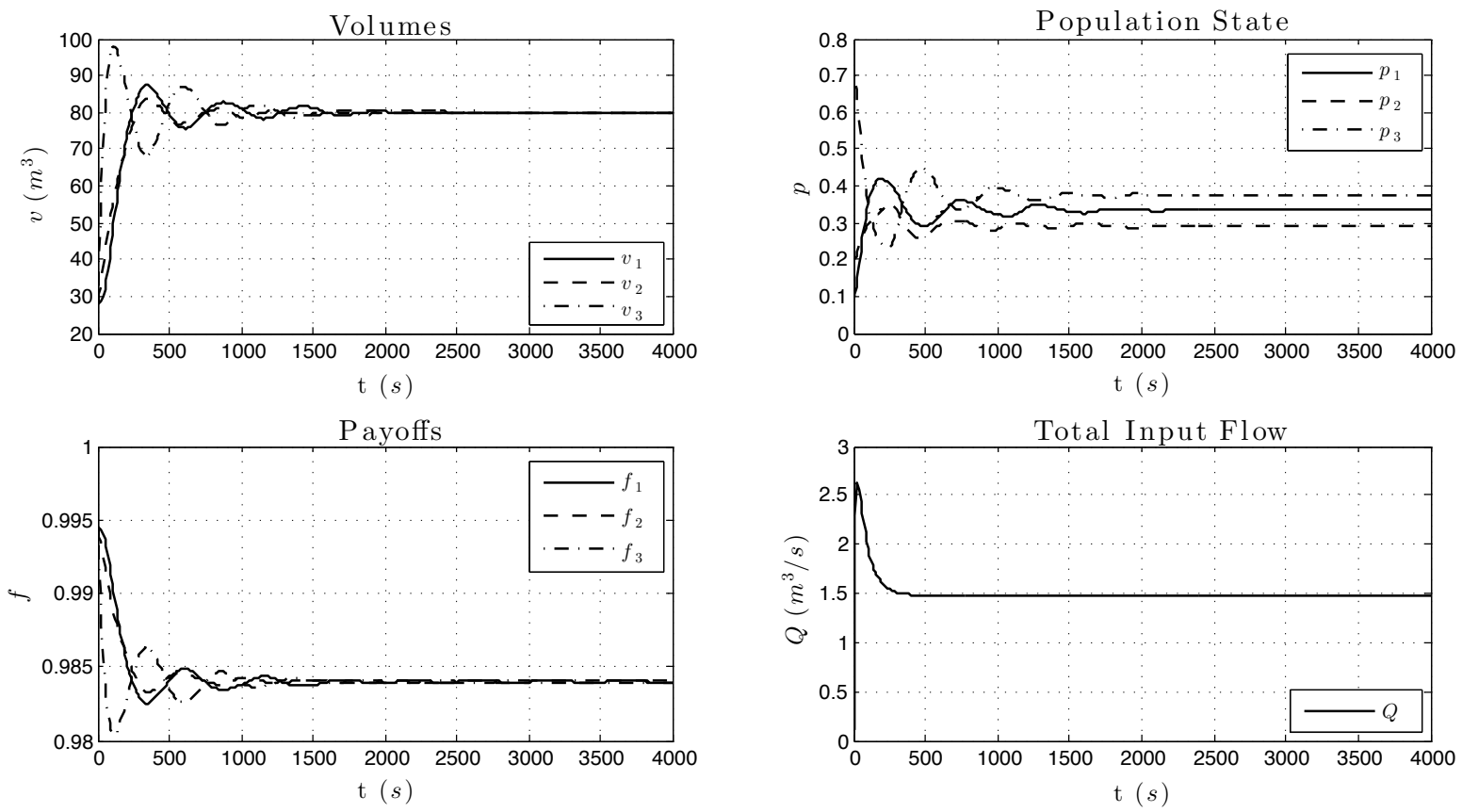

Figure 8.2: Tank system and the BNN dynamic, with initial condition 1. 
Also for the Smith dynamics we have:
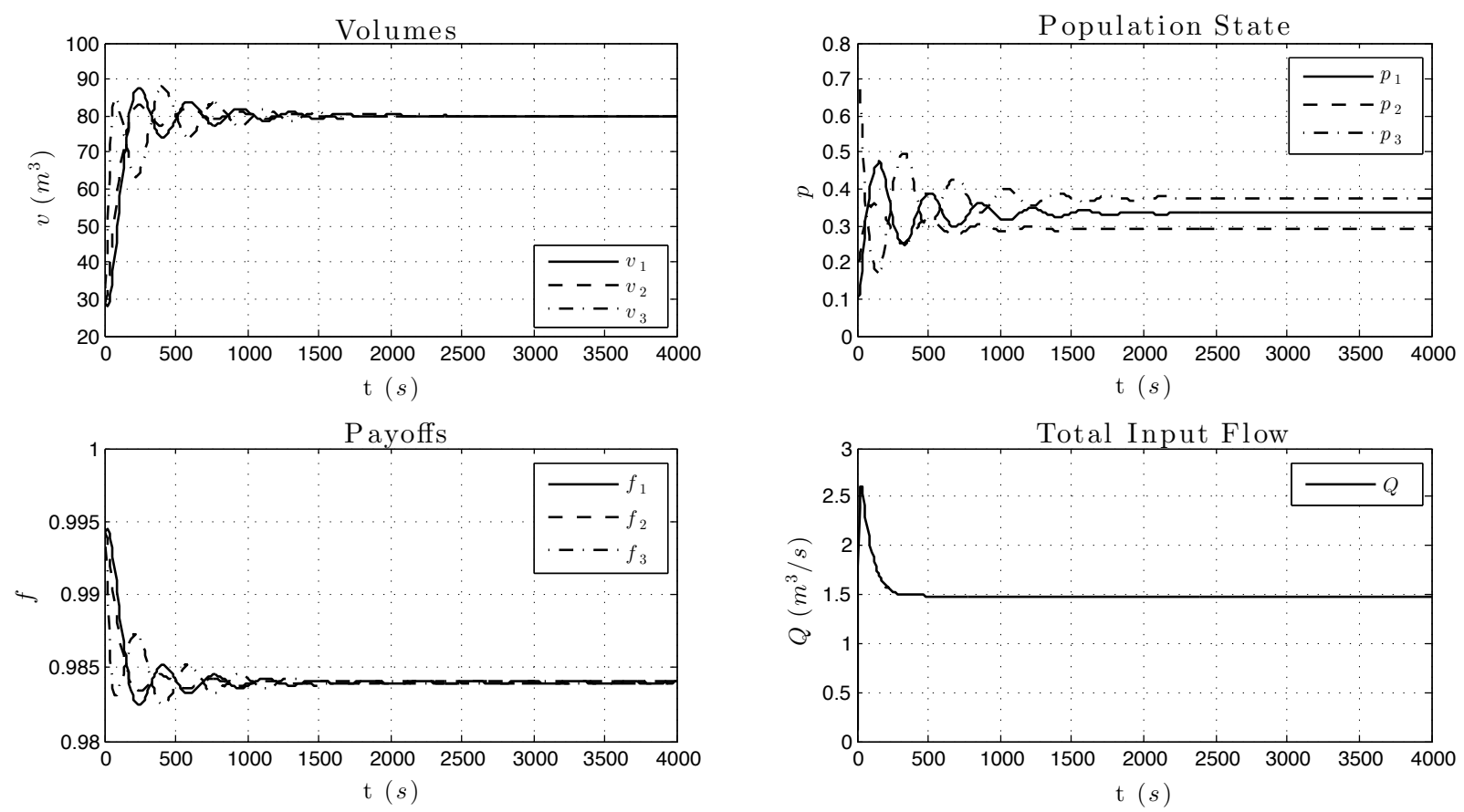

Figure 8.3: Tank system and the Smith dynamic, with initial condition 1.

As can be seen from the plots, the tank system in connection with the BNN or Smith dynamics is faster than the Replicator Dynamic in converging to the $N E$. In all of the results, the goal, which is converging to the desired $N E$, is achieved and the players are getting the maximum possible payoff in a way that all of them are satisfied with their payoffs. In the next section, we will see that by choosing different initial conditions, the Replicator Dynamic shows some unstable behaviour while the BNN and Smith dynamics preserve their desirable $N E$ seeking.

\subsection{Convergence to the Interior $N E$}

In this section, we change the initial conditions and run the simulations again. First, we plot the results of the negative feedback interconnection of the WDS with the Replicator Dynamic. Recalling from Chapter 2, we know that the Replicator Dynamics admits 
non-Nash equilibrium points. The plots below show the result of convergence of the Replicator Dynamics to the equilibrium points that are not the desired $N E$ 's. We change the initial conditions of the tank system from Table 8.2 to $v(0)=[1200,300,30]^{T}$ (initial $^{2}$ condition 2), we get:
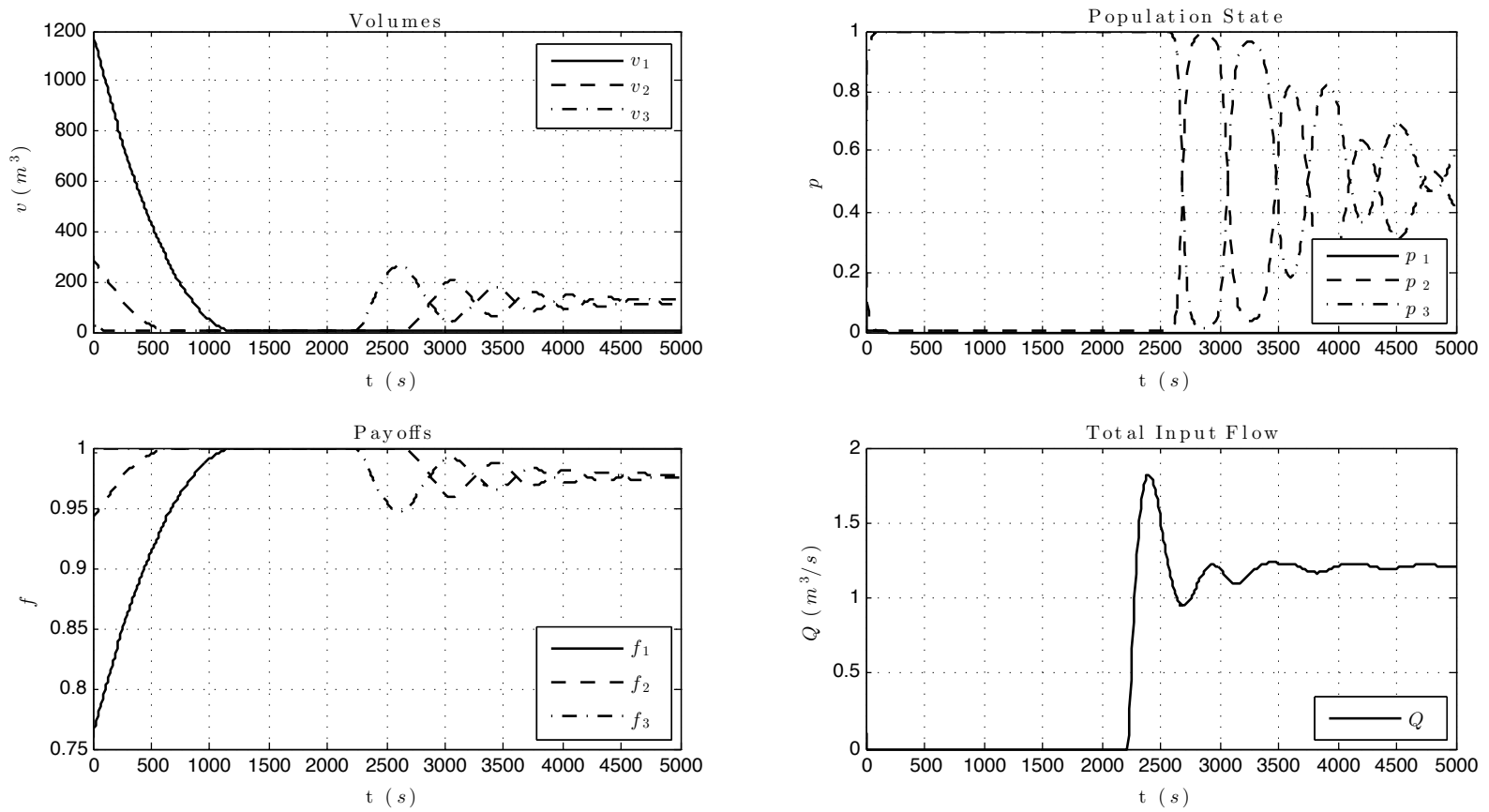

Figure 8.4: Tank system and the Replicator Dynamic, with initial condition 2.

It is clear from the plots that the first tank remains empty while the total input flow is being distributed between tanks 2 and 3. Furthermore, due to the payoff configuration, empty tanks have higher payoffs, therefore, it is desirable for the agents to switch from their strategies to tank 1 . However, since $p_{1}=0$ is one of the equilibrium points, the Replicator Dynamic will remain at this point. This means that the agents playing tank 2 and 3 are not satisfied with their choices. Since having an empty tank is not desired in this problem, we can conclude that due to the (NS) property, the Replicator Dynamic is not a suitable controller for our system. So we employ the BNN and Smith dynamics as alternatives to control the WDS. The following figures show the BNN and Smith dynamics results for the second set of initial conditions. 

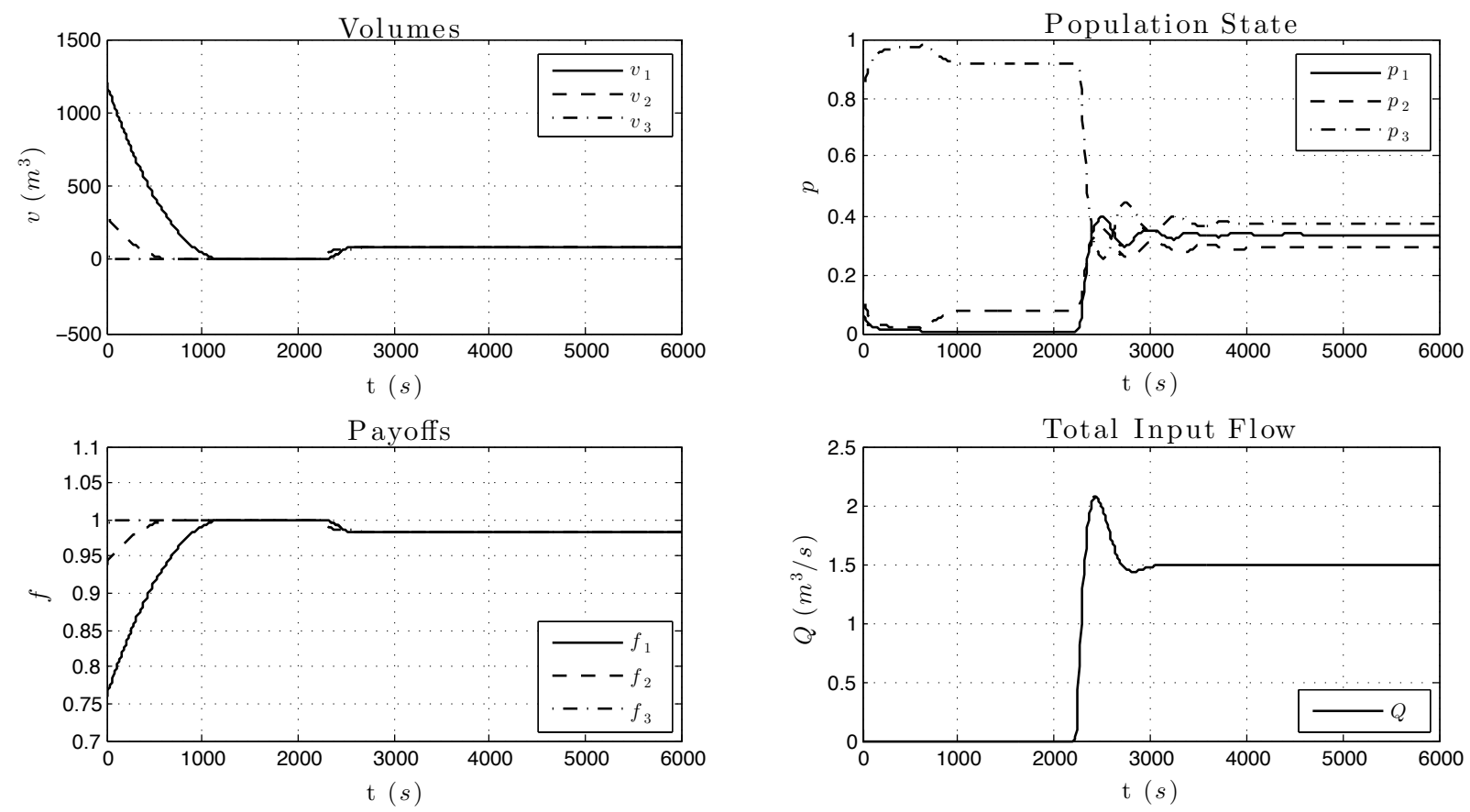

Figure 8.5: Tank system and the BNN dynamic, with initial condition 2.

The figures below illustrate the simulation results of the Smith dynamic to initial condition 2:
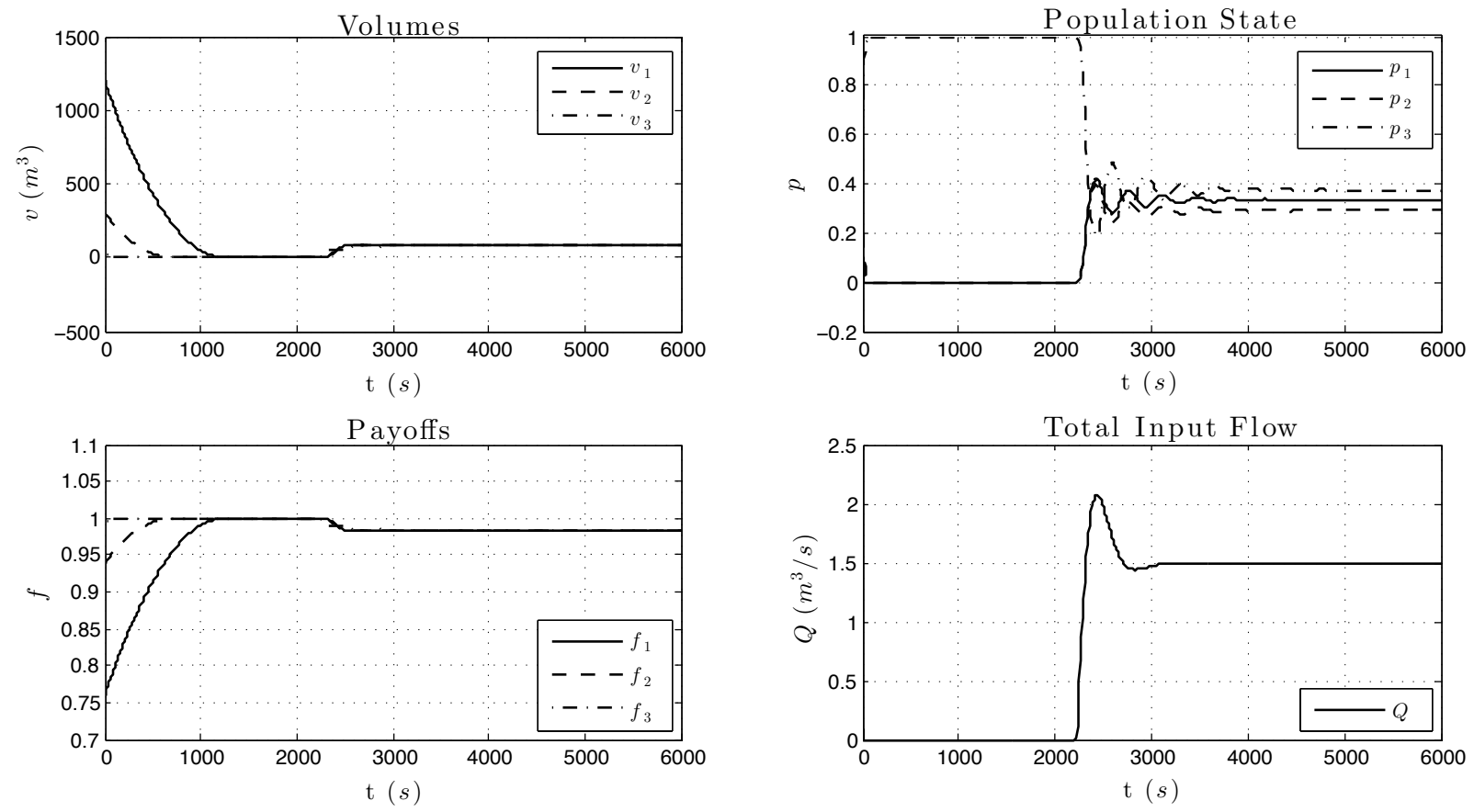

Figure 8.6: Tank system and the Smith dynamic, with initial condition 2. 
Figures 8.5 and 8.6 show that the problem of converging to non-Nash equilibrium points is solved by replacing the Replicator Dynamic with the BNN or Smith dynamic. It is illustrated in these figures that the system stabilizes and converges to the desirable $N E$ 's regardless of choosing different initial conditions.

In the next section, the effects of the total input flow controller on the system will be discussed.

\subsection{Disturbance Rejection}

In this part, we disturb the system with an external signal. We consider $u$, demonstrated in Figure 3.2, as an extra water consumption or malfunctioning in the water supply system; generally, any kinds of step-like function that can be applied to the WDS.

To analyze the behaviour of the system, first we neglect the dynamics of the total input flow controller and apply the disturbance. In fact, we assume that $Q$ is static. Next, we apply the disturbance while the dynamic of $Q$ (equation (4.30) ) is affecting the feedback system. And in the third case, the system is characterized when together, the integrator and the total input flow controller are controlling the system (see Chapter 6).

The following figure shows a simple step-like disturbance that will be applied to the system for the period of 3000 seconds:

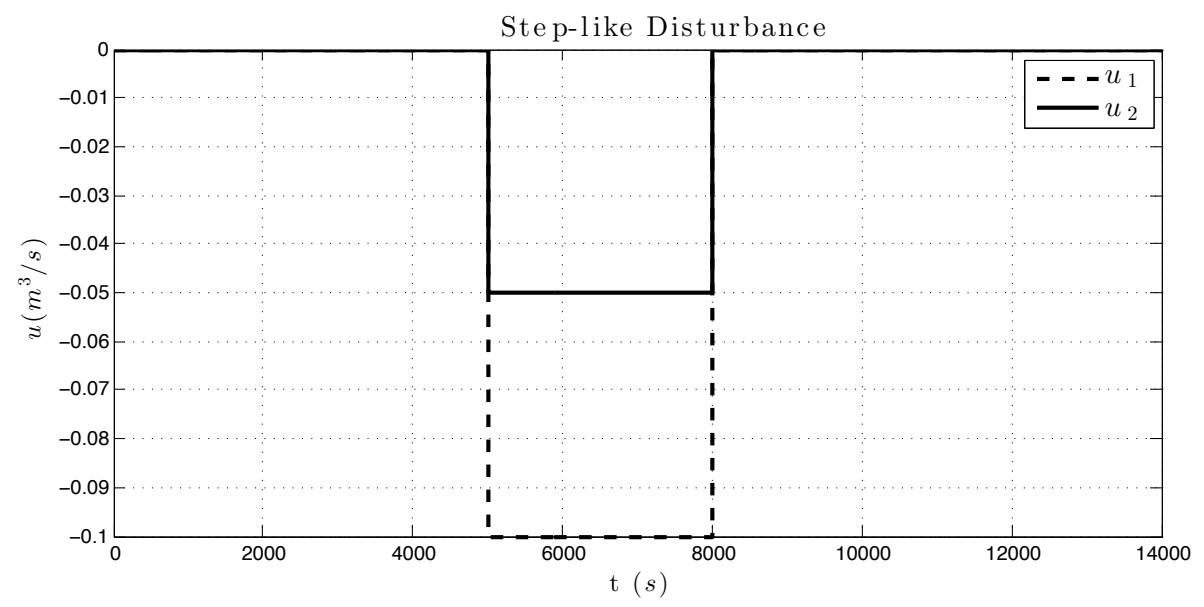

Figure 8.7: Step-like disturbance. 
We apply disturbances to the first and the second tanks but leave the third tank being drained under the atmospheric pressure to see the effects of the disturbance on nondisturbed tanks while others are disturbed. In the following figures, different responses of the tank system, which is connected to the BNN dynamic, to the disturbance are presented.

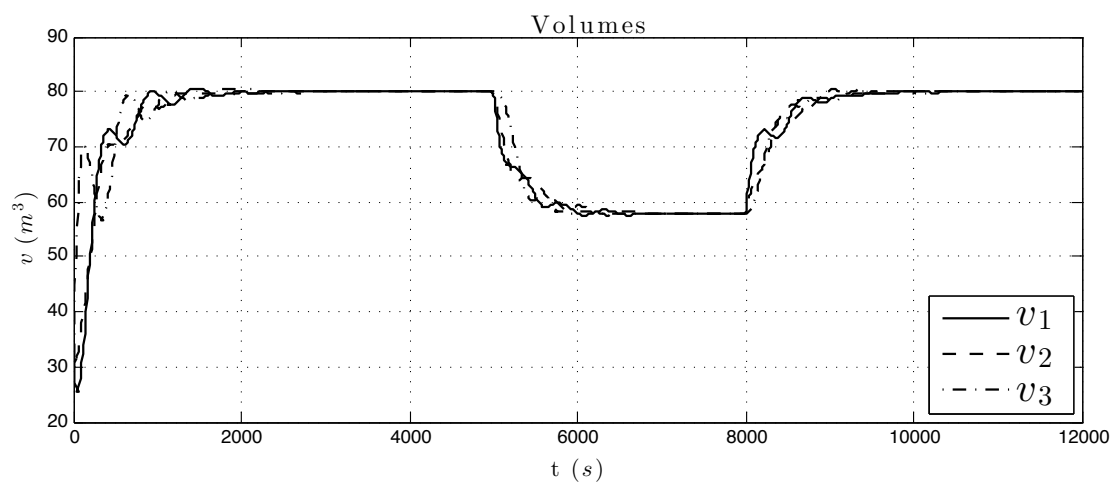

(a): Tank volumes with static Q.

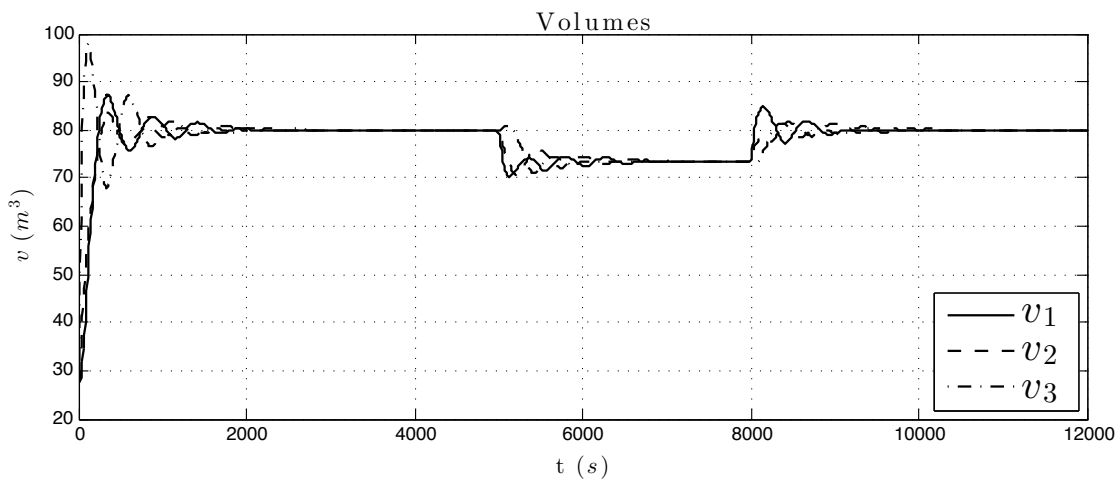

(b): Tank volumes with dynamic Q.

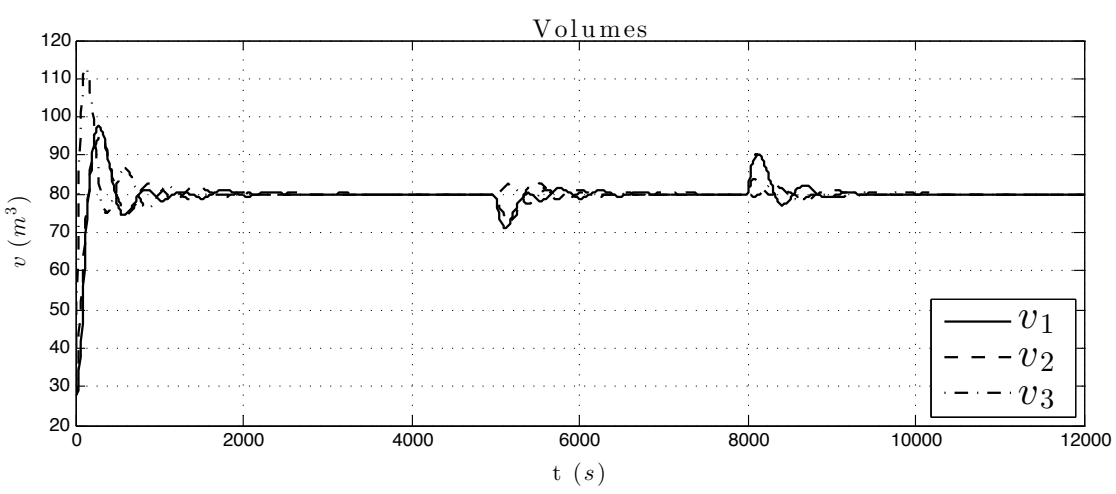

(c): Tank volumes with dynamic $\mathrm{Q}$ and the integrator.

Figure 8.8: Tank system and the BNN dynamic. 
In Figure 8.8 $\mathrm{a}$, it is assumed that $Q$ is a constant parameter while in Figure (b), $Q$ has dynamic which is described by equation 4.30). In Figure (c), an integrator, shown in Figure 6.1, is added to the system. It is clear that the response of the system in converging to the $N E$, even in the presence of the disturbance, has been dramatically improved. In the figures below, compensation of the system in supplying appropriate input flow is demonstrated.

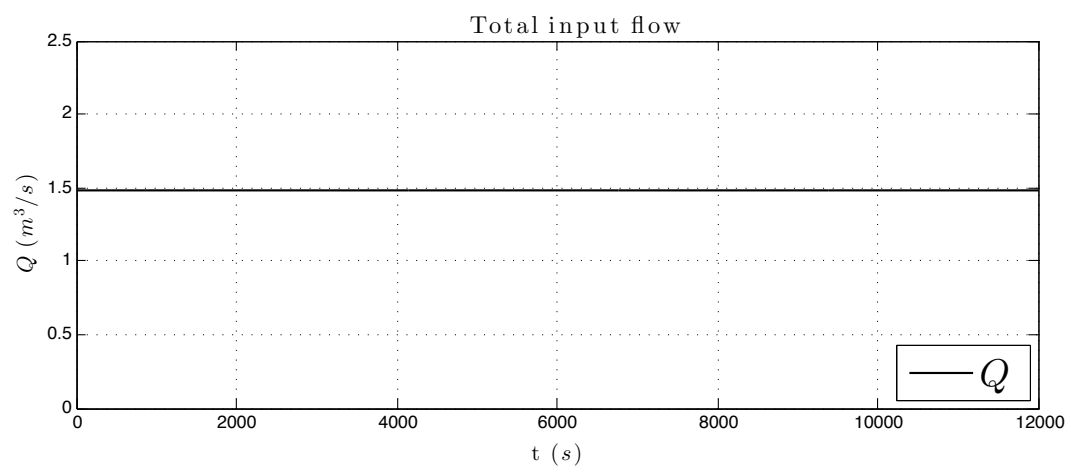

(a): Static Q.

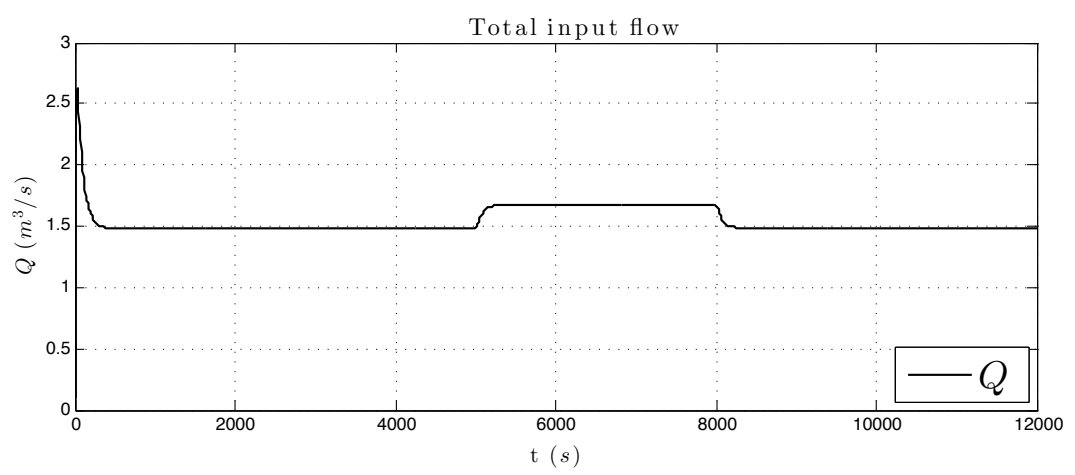

(b): Dynamic Q.

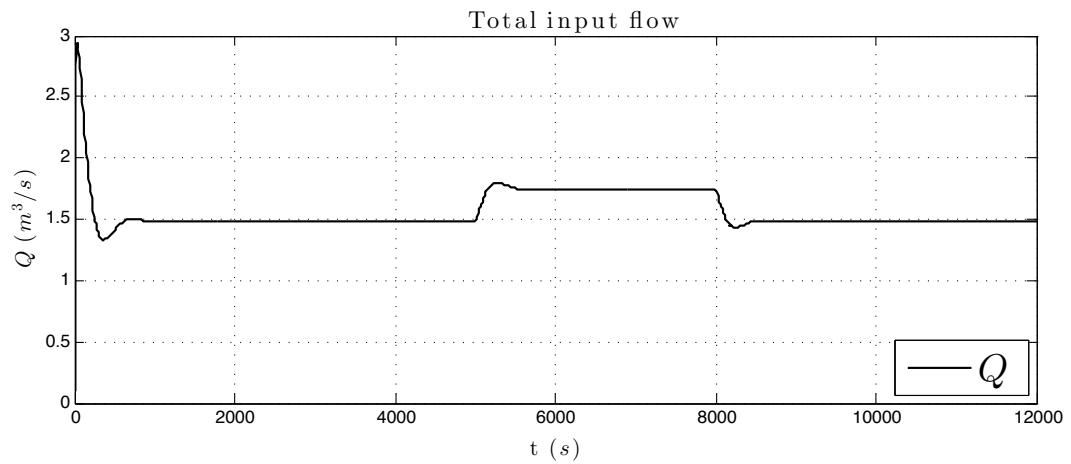

(c): Dynamic Q and the integrator.

Figure 8.9: Total input flow of the tank system in connection with the BNN dynamic. 
In the following plots, the simulation results of the WDS and the Smith dynamic with the same configuration used in Figure 8.8 have been provided.

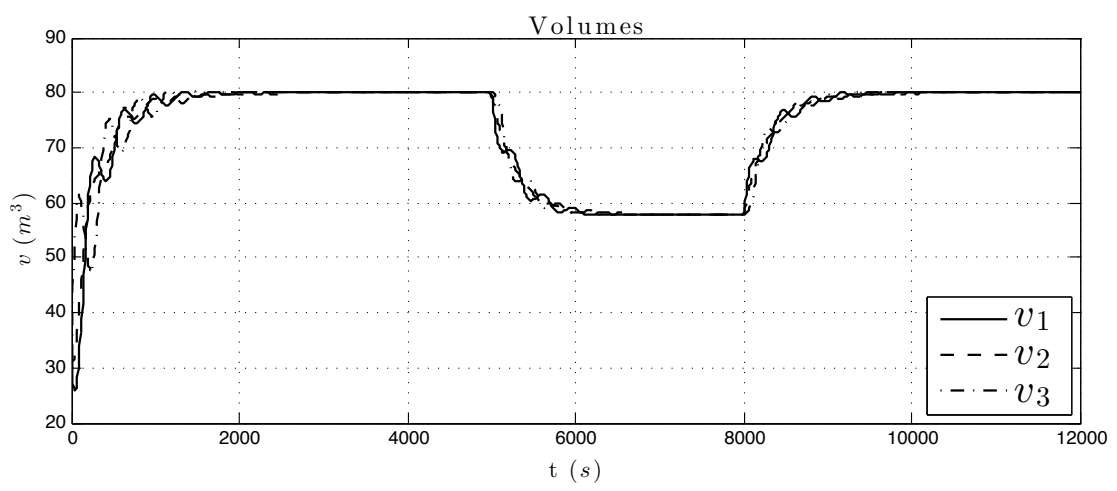

(a): Tank volumes with static Q.

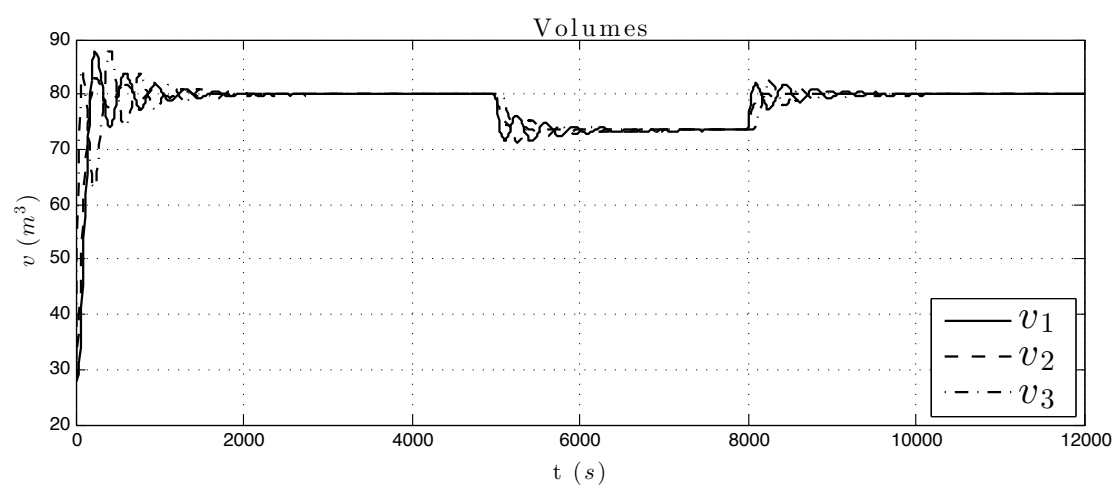

(b): Tank volumes with dynamic Q.

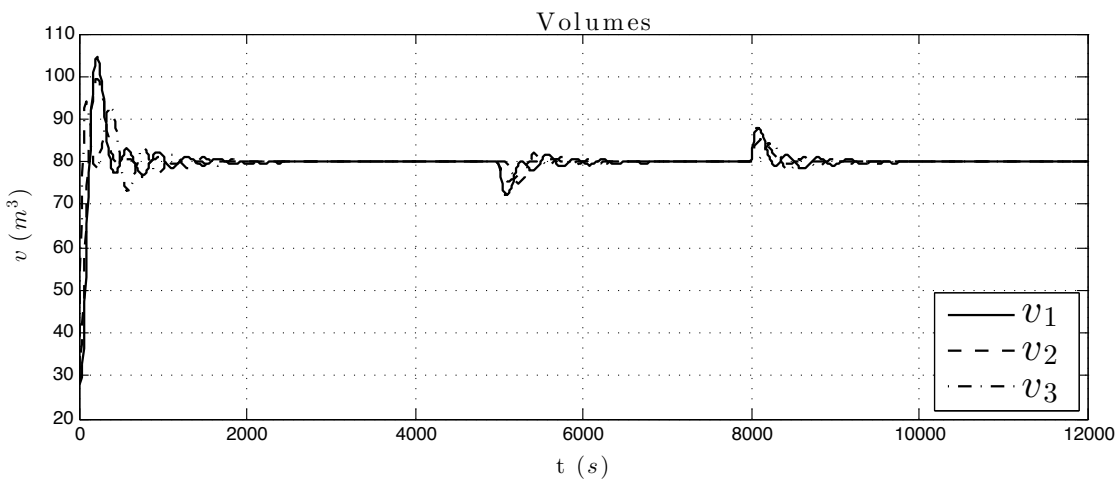

(c): Tank volumes with dynamic $\mathrm{Q}$ and the integrator.

Figure 8.10: Tank system and the Smith dynamic.

Next we present a more realistic output flow of the tanks which could be represented by the following plot: 


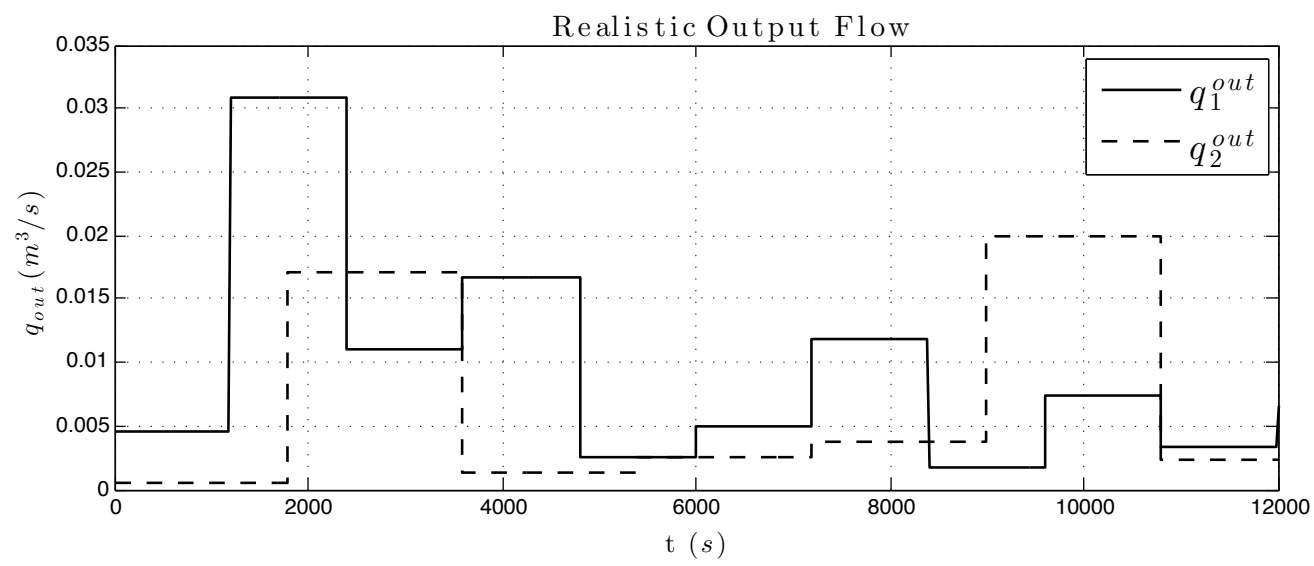

Figure 8.11: Stochastic rectangular disturbance pulses.

where the output flow of each tank is stochastic rectangular pulses. In fact, we consider changes in the pattern of the consumers' demands. Below, the response of the system to this type of disturbance is presented.

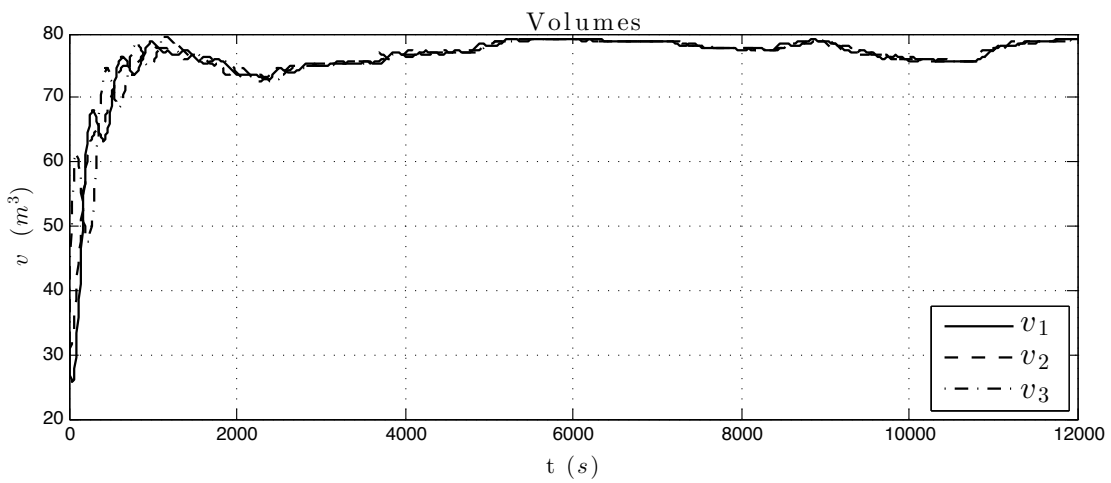

(a): Tank volumes with static Q.

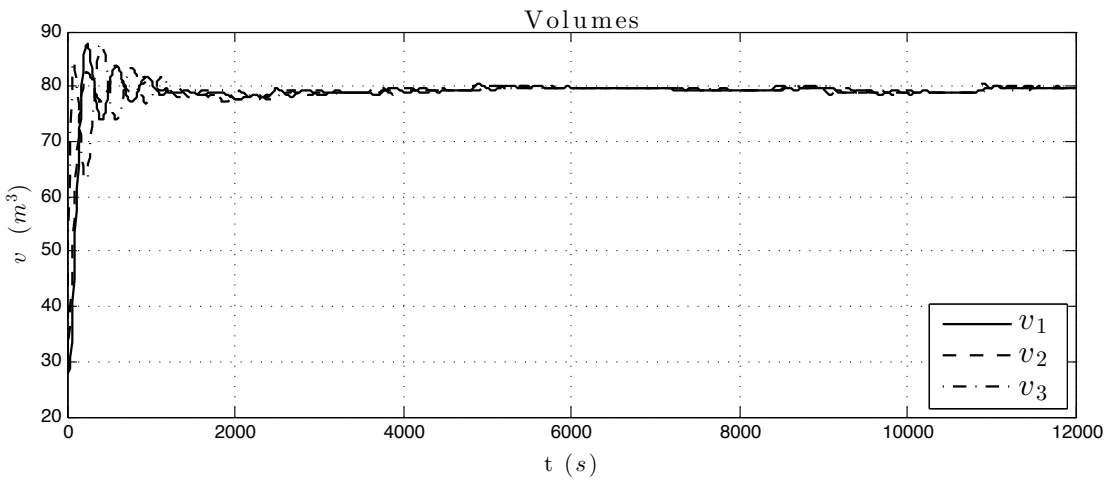

(b): Tank volumes with dynamic Q. 


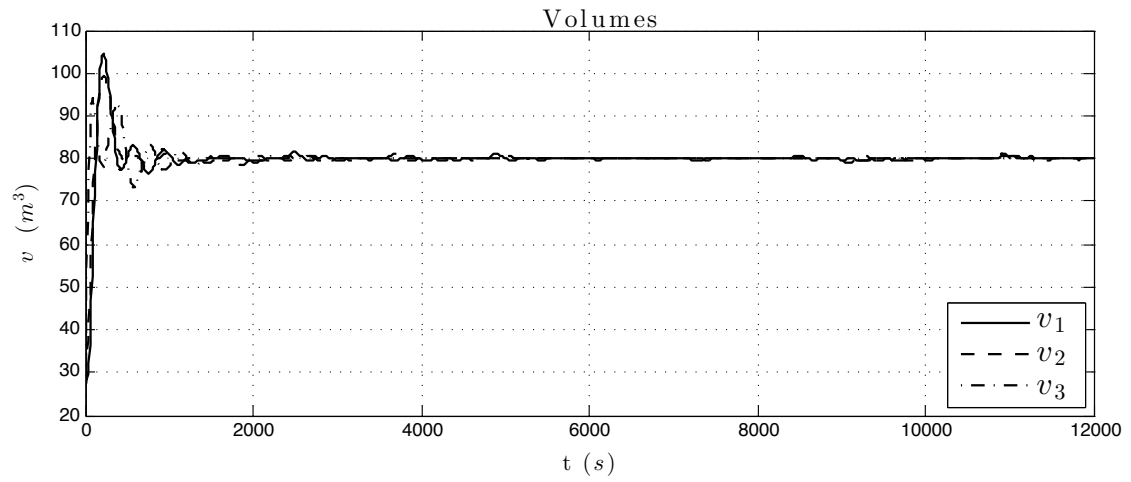

(c): Tank volumes with dynamic $\mathrm{Q}$ and the integrator.

Figure 8.12: Tank system and the Smith dynamic.

It is clear that the system with the integrator is robust to step-like disturbances. 


\section{Chapter 9}

\section{Conclusions}

\subsection{Concluding Remarks}

In this thesis, we considered control and optimization of distribution and supply of resources in resource allocation problems by using an evolutionary game-theoretic approach. This broad class of allocation problem that can include distribution of labor, water, fuel, energy, etc. has become a challenging problem for engineers from a simple problem of labor allocation to a complicated power distribution in wireless and optical networks.

The game-theoretic approach of evolutionary dynamics was first initiated by Nicanor Quijano in [29, 30], where the authors used the Replicator Dynamics for energy distribution, as well as [33, 34] in which a water distribution system was controlled by evolutionary dynamics. These works inspired us to use different classes of the evolutionary dynamics to solve the allocation problems; we considered the water distribution system as an example of a resource distribution problem to extend the existing results of [33, 29, 30, 34].

First, we formulated the WDS as a population game by defining strategies and associ-

ating a payoff function to each strategy. Then we used the Replicator Dynamic to control 
the game. Furthermore, we added the total input flow controller to enhance the behavior of the system. After interconnecting the system in a negative feedback interconnection, we characterized the stability of the system and presented sufficient conditions to ensure the stability of the negative feedback interconnection of the systems under study.

Second, after describing a potential problem of non-Nash convergence which could occur by using the Replicator Dynamic, we considered better-behaved revision protocols that led us to different classes of evolutionary dynamics with different properties. These dynamics are identified as the BNN and Smith dynamics that discard the non-Nash equilibrium points. Next, we turned to rejecting possible disturbances to the system, i.e., we looked for a subsystem to be added to the WDS to make the interconnection converge to the desirable Nash equilibria. For that, we formed and added an integrator to the total input flow controller of the system. With the added subsystem, the WDS became robust to step-like disturbances. This made the system converge to desired equilibrium points even while a disturbance was affecting the system.

As the last part of this thesis, to achieve globally asymptotic stability, we discarded the total input flow controller and only considered the BNN and Smith dynamics. In other words, we considered that total input flow as a constant that could be tuned externally. Furthermore, we extended the class of the population game that included the WDS. It was further demonstrated that under some conditions, the generalized class of the population games would globally converge to the desirable Nash equilibrium points.

Finally, we simulated the WDS with different controllers, the Replicator Dynamic, BNN and Smith dynamics. The problem of non-Nash convergence was shown and the solution was presented as well. Also the effects of the total input flow controller on the system responses were reviewed. Furthermore, a more realistic type of the output was examined and the results were illustrated. 


\subsection{Future Work}

Some issues remain open in thesis that we will address and suggest a solution approach for future research in resource allocation problems. In the following, the directions of the future work are provided:

- Resource allocation problems with strictly limited resources:

In these problems, resources are limited and overflows and underflows in resource usage are not allowed. For example in the WDS, unlike the assumptions of this thesis, neither the long term run overflows nor the instant overflows are allowed. To model the constraints of the resource, optimization methods for constrained problems can be utilized. Two general methods of indirect penalty approach and the Lagrangian pricing approach can be used to consider these constraints in the model of the total input flow controller.

- Limited access to provide necessary information to the controllers:

In these cases, we consider that full information about the strategies and the agents of the population game is not available to the controllers. Initial works have shown that the evolutionary dynamic along with the total input flow controller in the water distribution system still shows stable behavior despite having a lack of data. Also, having errors in providing essential information can be modeled in this case.

- Uncontrollable flow of the resource in distribution problems:

Consider the WDS when the total input flow $Q$, is not controllable, i.e., it is a fixed parameter. In this case, if the resource is more than the needed amount, the evolutionary dynamic will fail in delivering a desirable behavior to the system. One of the solutions to this problem is defining an artificial strategy with a specific payoff function. By associating appropriate payoff to the artificial strategy, the evolutionary dynamic will be able to dedicate the extra resource to the new strategy and make the WDS converge to the desired equilibrium points. Similarly, the 
problem of shortage of resources could also be investigated. 


\section{Part IV}

Appendices 


\section{Appendix A}

\section{Mathematical Theorems and Proofs}

\section{A.1 Positive Definite Symmetric Matrices}

Lemma A.1. The following $(N+1) \times(N+1)$ symmetric matrix:

$$
\mathcal{M}=\left[\begin{array}{cccc}
a & b_{1} & \ldots & b_{N} \\
b_{1} & c 1 & \ldots & 0 \\
\vdots & \vdots & \ddots & \vdots \\
b_{N} & 0 & \ldots & c_{N}
\end{array}\right]
$$

is positive definite if and only if $\operatorname{det} \mathcal{M}>0$, i.e.,

$$
a>\sum_{i=1}^{N} \frac{b_{i}^{2}}{c_{i}}
$$

Proof: In a positive definite matrix, all of the principal leading minors are strictly positive definite. Consider the principal leading minors of the matrix $\mathcal{M}$ as follows:

$$
M_{1}=a, \quad M_{2}=\operatorname{det}\left[\begin{array}{cc}
a & b_{1} \\
b_{1} & c_{1}
\end{array}\right], \ldots, \quad M_{N+1}=\operatorname{det} \mathcal{M}
$$


By calculating the above principal leading minors, it can simply be seen that if the following holds for the last minor:

$$
\operatorname{det} \mathcal{M}=M_{N+1}=a \prod_{i=1}^{N} c_{i}-\sum_{j=1}^{N}\left(b_{j}^{2} \prod_{k=1 \neq j}^{N} c_{k}\right)>0
$$

then all other minors are positive definite and the proof is immediate.

\section{A.2 Proof of Theorem 5.3}

Consider the following storage function that is introduced in Theorem 5.3 ;

$$
\mathcal{V}=\frac{v_{m}}{r} \sum_{i=1}^{N}\left(\Delta x_{2 i}+x_{2 i}^{*}\right)\left(\sum_{j=1}^{N} \int_{0}^{\left(\Delta x_{3}+x_{3}\right)^{1 / 2}\left(f_{j}-f_{i}\right)}[s]_{+} d s\right)+\frac{1}{2} \sum_{i=1}^{N}\left(\Delta \dot{x}_{1 i}\right)^{2}+\frac{m}{2 k \beta}\left(\Delta \dot{x}_{3}\right)^{2}
$$

Arguments in Section 5.2.1 for equation 5.22 prove that the above storage function is positive definite. By taking the derivative along the trajectories of equation (5.29), we get

$$
\dot{\mathcal{V}}=\sum_{j=1}^{N} \frac{\partial \mathcal{V}}{\partial \Delta x_{1 j}} \Delta \dot{x}_{1 j}+\sum_{j=1}^{N} \frac{\partial \mathcal{V}}{\partial \Delta x_{2 j}} \Delta \dot{x}_{2 j}+\frac{\partial \mathcal{V}}{\partial \Delta x_{3}} \Delta \dot{x}_{3}
$$

The first term of equation A.1 is given by

$$
\begin{aligned}
\sum_{j=1}^{N} \frac{\partial \mathcal{V}}{\partial \Delta x_{1 j}} \Delta \dot{x}_{1 j}= & \left(\Delta x_{3}+x_{3}^{*}\right) \sum_{j=1}^{N}\left[\left(\Delta x_{2 j}+x_{2 j}^{*}\right) \sum_{i=1}^{N} \rho_{j i}-\sum_{i=1}^{N}\left(\Delta x_{2 i}+x_{2 i}^{*}\right) \rho_{i j}\right] \Delta \dot{x}_{1 i} \\
& -\frac{1}{2} \sum_{j=1}^{N} c_{j} \frac{\left(\Delta \dot{x}_{1 j}\right)^{2}}{\sqrt{\Delta x_{1 j}+x_{1}^{*}}}-m \Delta \dot{x}_{3} \sum_{j=1}^{N} \Delta \dot{x}_{1 j} \\
= & -\left(\Delta x_{3}+x_{3}^{*}\right) \sum_{j=1}^{N} \Delta \dot{x}_{2 j} \Delta \dot{x}_{1 j}-\frac{1}{2} \sum_{j=1}^{N} c_{j} \frac{\left(\Delta \dot{x}_{1 j}\right)^{2}}{\sqrt{\Delta x_{1 j}+x_{1}^{*}}}-m \Delta \dot{x}_{3} \sum_{j=1}^{N} \Delta \dot{x}_{1 j} .
\end{aligned}
$$


The second term of equation A.1 is the following:

$\sum_{j=1}^{N} \frac{\partial \mathcal{V}}{\partial \Delta x_{2 j}} \Delta \dot{x}_{2 j}=\frac{v_{m}}{r} \sum_{i=1}^{N} \Delta \dot{x}_{2 i}\left(\sum_{j=1}^{N} \int_{0}^{\left(\Delta x_{3}+x_{3}\right)^{1 / 2}\left(f_{j}-f_{i}\right)}[s]_{+} d s\right)+\sum_{j=1}^{N}\left(\Delta x_{3}+x_{3}^{*}\right) \Delta \dot{x}_{2 j} \Delta \dot{x}_{1 j}$

where the first term of the above equation is negative definite due to the discussion for equation 7.13$)$. And for the third term we have

$$
\begin{aligned}
\frac{\partial \mathcal{V}}{\partial \Delta x_{3}} \Delta \dot{x}_{3}= & \frac{v_{m}}{2 r} \Delta \dot{x}_{3} \sum_{j=1}^{N}\left(\Delta x_{2 j}+x_{2 j}^{*}\right) \sum_{i=1}^{N}\left(f_{i}-f_{j}\right)\left[f_{i}-f_{j}\right]_{+} \\
& +\Delta \dot{x}_{3} \sum_{j=1}^{N}\left(\Delta x_{2 j}+x_{2 j}^{*}\right) \Delta \dot{x}_{1 j}-m \frac{\alpha}{\beta \hat{Q}}\left(\Delta \dot{x}_{3}\right)^{2} .
\end{aligned}
$$

Then, the sum of all terms of A.1 in terms of quadratic form satisfies

$$
\begin{aligned}
\dot{\mathcal{V}}= & -\left[\begin{array}{ll}
\Delta \dot{x}_{3} & \Delta \dot{x}_{1}^{T}
\end{array}\right] \mathcal{A}\left[\begin{array}{c}
\Delta \dot{x}_{3} \\
\Delta \dot{x}_{1}
\end{array}\right]+\frac{v_{m}}{r} \sum_{i=1}^{N} \Delta \dot{x}_{2 i}\left(\sum_{j=1}^{N} \int_{0}^{\left(\Delta x_{3}+x_{3}\right)^{1 / 2}\left(f_{j}-f_{i}\right)}[s]_{+} d s\right) \\
& +\frac{v_{m}}{2 r} \Delta \dot{x}_{3} \sum_{j=1}^{N}\left(\Delta x_{2 j}+x_{2 j}^{*}\right) \sum_{i=1}^{N}\left(f_{i}-f_{j}\right)\left[f_{i}-f_{j}\right]_{+} \\
= & \theta_{8}+\theta_{9}+\theta_{10}
\end{aligned}
$$

where $\mathcal{A}$ is the same matrix as in equation 5.25 and

$$
\begin{aligned}
\theta_{8} & =\frac{v_{m}}{r} \sum_{i=1}^{N} \Delta \dot{x}_{2 i}\left(\sum_{j=1}^{N} \int_{0}^{\left(\Delta x_{3}+x_{3}\right)^{1 / 2}\left(f_{j}-f_{i}\right)}[s]_{+} d s\right), \\
\theta_{9} & =\frac{v_{m}}{2 r} \Delta \dot{x}_{3} \sum_{j=1}^{N}\left(\Delta x_{2 j}+x_{2 j}^{*}\right) \sum_{i=1}^{N}\left(f_{i}-f_{j}\right)\left[f_{i}-f_{j}\right]_{+}, \\
\theta_{10} & =-\left[\begin{array}{ll}
\Delta \dot{x}_{3} & \Delta \dot{x}_{1}^{T}
\end{array}\right] \mathcal{A}\left[\begin{array}{c}
\Delta \dot{x}_{3} \\
\Delta \dot{x}_{1}
\end{array}\right] .
\end{aligned}
$$

Arguments in [20] and also in Section 7.1 for equation (7.13) of this thesis, establish that $\theta_{8}$ is negative definite while $\theta_{9}$ depends on the sign of $\Delta \dot{x}_{3}$. And for the last term, 
$\theta_{10}$ is negative definite if and only if the condition presented in equation (5.26) holds. Therefore, by following the same procedure utilized in Theorem 5.2 , one can find a D.o.A such that

$$
\left|\theta_{9}\right|<\left|\theta_{8}+\theta_{10}\right|
$$

Thus,

$$
\dot{\mathcal{V}}=\theta_{8}+\theta_{9}+\theta_{10}<0
$$

and the negative feedback interconnection of the game dynamic and the Smith dynamic is locally asymptotically stable.

\section{A.3 Domain of Attraction}

Choosing different values of $d$, utilized in the proof of Theorem 5.1, could change the Domain of Attraction (D.o.A) and as a consequence, the stability characteristics of the feedback interconnected system. In the following discussion, using different values of $d$ are analyzed and the domain of attraction is derived at the end of this part. First, we rewrite the equations of 5.10 to get the following form:

$$
\begin{aligned}
& \theta_{1}=\left(\Delta x_{1}\right)^{T} \mathbb{I} m, \\
& \theta_{2}=-\frac{\alpha}{\beta \hat{Q}} \Delta x_{3}^{2}, \\
& \theta_{3}=\frac{1}{\Delta x_{3}+x_{3}^{*}}\left(\Delta x_{1}\right)^{T} m, \\
& \theta_{4}=\frac{\Delta x_{3}}{\Delta x_{3}+x_{3}^{*}}\left(\Delta x_{1}\right)^{T} x_{2}^{*}-\frac{\Delta \dot{x}_{3}}{2\left(\Delta x_{3}+x_{3}^{*}\right)^{2}}\left(\Delta x_{1}\right)^{T} \Delta x_{1},
\end{aligned}
$$


where $m=\left[m_{1}, \ldots, m_{N}\right]^{T}$ and $m_{i}=c_{i}\left(\sqrt{x_{1}^{*}}-\sqrt{\Delta x_{1 i}+x_{1}^{*}}\right)$ and $\mathbb{I}$ is an all-ones $N \times N$ matrix. Now by substituting (4.30) in the equation of $\theta_{4}$, we get

$$
\theta_{4}=\frac{\Delta x_{3}}{\Delta x_{3}+x_{3}^{*}}\left(\Delta x_{1}\right)^{T} x_{2}^{*}+\left(\frac{k \alpha}{2 \hat{Q}} \frac{\Delta x_{3}}{\left(\Delta x_{3}+x_{3}^{*}\right)^{2}}+\frac{k \beta}{2} \frac{\left(\Delta x_{1}\right)^{T} \mathbf{1}}{\left(\Delta x_{3}+x_{3}^{*}\right)^{2}}\right)\left(\Delta x_{1}\right)^{T} \Delta x_{1}
$$

By taking the Euclidean norm of both sides of equations A.4 and (A.5), and using the Cauchy-Schwarz inequality 1 , we get

$$
\begin{gathered}
\left|\theta_{1}\right| \leqslant\left\|\Delta x_{1}\right\|_{2}\|\mathbb{I}\|_{2}\|m\|_{2}, \\
\left|\theta_{2}\right|=\frac{\alpha}{\beta \hat{Q}}\left|\Delta x_{3}\right|^{2} \\
\left|\theta_{3}\right| \leqslant \frac{1}{\left|\Delta x_{3}+x_{3}^{*}\right|}\left\|\Delta x_{1}\right\|_{2}\|m\|_{2}, \\
\left|\theta_{4}\right| \leqslant \frac{\left|\Delta x_{3}\right|}{\left|\Delta x_{3}+x_{3}^{*}\right|}\left\|\Delta x_{1}\right\|_{2}\left\|x_{2}^{*}\right\|_{2}+\left(\frac{k \alpha}{2 \hat{Q}} \frac{\left|\Delta x_{3}\right|}{\left|\Delta x_{3}+x_{3}^{*}\right|^{2}}+\frac{k \beta}{2} \frac{\left\|\Delta x_{1}\right\|_{2}\|\mathbf{1}\|_{2}}{\left|\Delta x_{3}+x_{3}^{*}\right|^{2}}\right)\left\|\Delta x_{1}\right\|_{2}^{2} .
\end{gathered}
$$

For simplicity in notations, we drop the index of 2 from the Euclidean norms. Let us consider the following argument for $\|m\|$ :

$$
\begin{aligned}
\|m\|^{2} & =\sum_{j=1}^{N} m_{j}^{2}=\sum_{j=1}^{N} c_{j}^{2}\left(2 x_{1}^{*}+\Delta x_{1 j}-2 \sqrt{x_{1}^{*}\left(\Delta x_{1 j}+x_{1}^{*}\right)}\right) \\
& \leqslant \sum_{j=1}^{N} c_{j}^{2}\left(2 x_{1}^{*}+\Delta x_{1 j}\right)=\left[\begin{array}{lll}
c_{1}^{2} & \ldots & c_{N}^{2}
\end{array}\right]^{T}\left(2 x_{1}^{*} \mathbf{1}+\Delta x_{1}\right) \\
& \leqslant 2 x_{1}^{*}\left(c_{1}^{2}+\cdots+c_{N}^{2}\right)+\left\|\left[\begin{array}{lll}
c_{1}^{2} & \ldots & c_{N}^{2}
\end{array}\right]\right\| \cdot\left\|\Delta x_{1}\right\| \\
& =2 x_{1}^{*} \bar{c}+\overline{\bar{c}}\left\|\Delta x_{1}\right\| .
\end{aligned}
$$

So we have

$$
\|m\| \leqslant \sqrt{2 x_{1}^{*} \bar{c}+\bar{c}\left\|\Delta x_{1}\right\|} \leqslant \sqrt{2 x_{1}^{*} \bar{c}}+\sqrt{\overline{\bar{c}}\left\|\Delta x_{1}\right\|},
$$

where $\bar{c}=c_{1}^{2}+\cdots+c_{N}^{2}$ and $\overline{\bar{c}}=\left\|\left[\begin{array}{lll}c_{1}^{2} & \ldots & c_{N}^{2}\end{array}\right]\right\|$.

\footnotetext{
${ }^{1}$ Cauchy-Schwarz inequality: $|x \cdot y| \leqslant\|x\|_{2}\|y\|_{2}$.
} 
Now by using (A.8) in equations of A.6 and A.7 and simplifying them, the inequality of $\theta_{1}$ will be of form

$$
\begin{aligned}
\left|\theta_{1}\right| & \leqslant\left\|\Delta x_{1}\right\|\|\mathbb{I}\|\|m\| \leqslant N\left\|\Delta x_{1}\right\|\left(\sqrt{2 x_{1}^{*} \bar{c}}+\sqrt{\overline{\bar{c}}\left\|\Delta x_{1}\right\|}\right) \\
& =a_{1}\left\|\Delta x_{1}\right\|+a_{2}\left\|\Delta x_{1}\right\|^{3 / 2}
\end{aligned}
$$

where $a_{1}=N \sqrt{2 x_{1}^{*} \bar{c}}$ and $a_{2}=N \sqrt{\overline{\bar{c}}}$. Notice that $\|\mathbb{I}\|=N$. For $\theta_{3}$, the inequality takes the following form:

$$
\begin{aligned}
\left|\theta_{3}\right| & \leqslant \frac{1}{\left|\Delta x_{3}+x_{3}^{*}\right|}\left\|\Delta x_{1}\right\|\|m\| \leqslant \frac{1}{\left|\Delta x_{3}+x_{3}^{*}\right|}\left\|\Delta x_{1}\right\|\left(\sqrt{2 x_{1}^{*} \bar{c}}+\sqrt{\overline{\bar{c}}\left\|\Delta x_{1}\right\|}\right) \\
& =\frac{1}{N\left|\Delta x_{3}+x_{3}^{*}\right|}\left(a_{1}\left\|\Delta x_{1}\right\|+a_{2}\left\|\Delta x_{1}\right\|^{3 / 2}\right) .
\end{aligned}
$$

By following the same procedure for $\theta_{4}$, we get the last inequality as follows:

$$
\left|\theta_{4}\right| \leqslant a_{3} \frac{\left|\Delta x_{3}\right|}{\left|\Delta x_{3}+x_{3}^{*}\right|}\left\|\Delta x_{1}\right\|+a_{4} \frac{\left|\Delta x_{3}\right|}{\left|\Delta x_{3}+x_{3}^{*}\right|^{2}}\left\|\Delta x_{1}\right\|^{2}+a_{5} \frac{1}{\left|\Delta x_{3}+x_{3}^{*}\right|^{2}}\left\|\Delta x_{1}\right\|^{3} .
$$

In the above inequality $a_{3}=\left\|x_{2}^{*}\right\|, a_{4}=\frac{k \alpha}{2 \hat{Q}}$ and $a_{5}=\frac{\sqrt{3}}{2} k \beta$. We also let $a_{6}=\frac{\alpha}{\beta \hat{Q}}$. Now going back to the inequality in equation (5.12) and considering the following inequality:

$$
d\left|\theta_{2}\right|-d\left|\theta_{1}\right|-\left|\theta_{3}\right| \leqslant d\left|\theta_{1}+\theta_{2}\right|-\left|\theta_{3}\right| \leqslant\left|d\left(\theta_{1}+\theta_{2}\right)+\theta_{3}\right|,
$$

if an appropriate $d$ is chosen such that

$$
\left|\theta_{4}\right|<d\left(\left|\theta_{2}\right|-\left|\theta_{1}\right|\right)-\left|\theta_{3}\right|
$$

obviously equation 5.12 will hold. By rewriting the above inequality we get

$$
\left|\theta_{4}\right|+\left|\theta_{3}\right|<d\left(\left|\theta_{2}\right|-\left|\theta_{1}\right|\right) .
$$


Recalling equation (A.6) for $\theta_{2}$ and equations A.9, A.10 and A.11, the above inequality takes the form of

$$
\begin{aligned}
\frac{1}{N}\left(a_{1}\left\|\Delta x_{1}\right\|\right. & \left.+a_{2}\left\|\Delta x_{1}\right\|^{3 / 2}\right)+\frac{\left\|\Delta x_{1}\right\|^{2}}{\left|\Delta x_{3}+x_{3}^{*}\right|}\left(a_{4}\left|\Delta x_{3}\right|+a_{5}\left\|\Delta x_{1}\right\|\right) \\
& +a_{3}\left|\Delta x_{3}\right|\left\|\Delta x_{1}\right\|<d\left|\Delta x_{3}+x_{3}^{*}\right|\left(a_{6}\left|\Delta x_{3}\right|^{2}-a_{1}\left\|\Delta x_{1}\right\|-a_{2}\left\|\Delta x_{1}\right\|^{3 / 2}\right) .
\end{aligned}
$$

Simplification in the above inequality leads us to the following

$$
\|\Delta x\|_{2}<\delta_{d}
$$

that indicates the D.o.A $\left(D_{\delta}\right)$. This inequality implies that the D.o.A could be affected by selecting different values for $d$. So if we choose a proper $d$ such that equation A.13) holds for a $\delta_{d}$ for the solutions of the system in the corresponding D.o.A, then the condition stated in equation 5.12 will be met. Therefore, equation 5.14 will be true. 


\section{Appendix B}

\section{Convex Optimization}

\section{B.1 Gradient Method}

In this section, we briefly review the gradient algorithm as one of the optimization methods in convex optimization.

Recent advances in the application of the convex optimization in industry, such as [24], have motivated a new look at classical optimization methods based on motion along the gradient [16]. In a simple optimization problem of form

$$
\underset{x}{\operatorname{minimize}} \mathcal{J}(x)
$$

we look for a point that minimizes $\mathcal{J}(x)$. The function $\mathcal{J}(x)$ could be considered as a cost function defined based on the parameters and variables of the system under study. We assume that the cost function is strictly convex in $x$, therefore, it can be shown that by moving in the opposite direction of the gradient of the cost function, i.e.,

$$
\dot{x}=\frac{d x}{d t}=-k \frac{d \mathcal{J}(x)}{d x},
$$


where $k$ is a positive constant, the solutions converge to a point where $\mathcal{J}(x)$ is minimized. This method is called the gradient algorithm that has been developed for a continuous-time domain. In other word, by solving the differential equation of (B.2), the solution arrives at the minimum of the corresponding cost function. The convergence and stability analysis of this method has been established in [15, 16, 23] where, for instance, Krasovskii's method is utilized to find an appropriate Lyapunov function. The reader can refer to [7, 9] for a thorough discussion around this topic in the convex optimization. 


\section{Bibliography}

[1] T. Alpcan, T. Başar, R. Srikant, and E. Altman, "CDMA uplink power control as a noncooperative game," Wireless Networks, vol. 8, no. 6, pp. 659-670, 2002.

[2] E. Altman and Z. Altman, "S-modular games and power control in wireless networks," IEEE Transactions on Automatic Control, vol. 48, no. 5, pp. 839-842, 2003.

[3] E. Altman, T. Basar, and R. Srikant, "Nash equilibria for combined flow control and routing in networks: Asymptotic behavior for a large number of users," IEEE Transactions on Automatic Control, vol. 47, no. 6, pp. 917-930, 2002.

[4] E. Altman, T. Boulogne, R. El-Azouzi, T. Jiménez, and L. Wynter, "A survey on networking games in telecommunications," Computers 83 Operations Research, vol. 33, no. 2, pp. 286-311, 2006.

[5] L. Araujo, H. Ramos, and S. Coelho, "Pressure control for leakage minimisation in water distribution systems management," Water Resources Management, vol. 20, no. 1, pp. 133-149, 2006.

[6] J. Bao and P. Lee, Process control: the passive systems approach. Springer, 2007.

[7] D. P. Bertsekas, Nonlinear programming. Athena Scientific, Nashua, 1999.

[8] P. R. Bhave and R. Gupta, "Optimal design of water distribution networks for fuzzy demands," Civil Engineering and Environmental Systems, vol. 21, no. 4, pp. $229-245,2004$. 
[9] S. P. Boyd and L. Vandenberghe, Convex optimization. Cambridge University Press, 2004.

[10] N. F. Britton, Essential mathematical biology. Springer, 2003.

[11] D. Broad, G. C. Dandy, and H. R. Maier, "Water distribution system optimization using metamodels," Journal of Water Resources Planning and Management, vol. 131, no. 3, pp. 172-180, 2005.

[12] L. M. Camarinha-Matos and F. J. Martinelli, "Application of machine learning in water distribution networks assisted by domain experts," Journal of Intelligent and Robotic Systems, vol. 26, no. 3-4, pp. 325-352, 1999.

[13] D. Famolari, N. Mandayam, D. Goodman, and V. Shah, "A new framework for power control in wireless data networks: Games, utility, and pricing," in Wireless Multimedia Network Technologies. Springer, 2002, pp. 289-309.

[14] X. Fan, T. Alpcan, M. Arcak, T. Wen, and T. Başar, "A passivity approach to game-theoretic CDMA power control," Automatica, vol. 42, no. 11, pp. 1837-1847, 2006.

[15] D. Feijer and F. Paganini, "Krasovskii's method in the stability of network control," in American Control Conference, 2009. ACC'09. IEEE, 2009, pp. 3292-3297.

[16] — "Stability of primal-dual gradient dynamics and applications to network optimization," Automatica, vol. 46, no. 12, pp. 1974-1981, 2010.

[17] M. J. Fox and J. S. Shamma, "Population games, stable games, and passivity," Games, vol. 4, no. 4, pp. 561-583, 2013.

[18] M. Fox and J. Shamma, "Population games, stable games, and passivity," in 2012 IEEE 51st Annual Conference on Decision and Control (CDC), Dec 2012, pp. 74457450. 
[19] J. Hofbauer, "Deterministic evolutionary game dynamics," Evolutionary Game Dynamics, pp. 61-79, 2011.

[20] J. Hofbauer and W. H. Sandholm, "Stable games and their dynamics," Journal of Economic Theory, vol. 144, no. 4, pp. 1665-1693, 2009.

[21] J. Hofbauer and K. Sigmund, Evolutionary games and population dynamics. Cambridge University Press, 1998.

[22] R. Johari and J. N. Tsitsiklis, "Efficiency loss in a network resource allocation game," Mathematics of Operations Research, vol. 29, no. 3, pp. 407-435, 2004.

[23] H. U. K. Arrow, L. Hurwicz, Studies in Linear and Non-Linear Programming. Stanford University Press, Stanford, California, 1958.

[24] F. P. Kelly, A. K. Maulloo, and D. K. Tan, "Rate control for communication networks: shadow prices, proportional fairness and stability," Journal of the Operational Research society, pp. 237-252, 1998.

[25] H. K. Khalil, Nonlinear systems, 3rd ed. Prentice Hall Upper Saddle River, 2002.

[26] L. Libman and A. Orda, "The designer's perspective to atomic noncooperative networks," IEEE/ACM Transactions on Networking, vol. 7, no. 6, pp. 875-884, 1999.

[27] K. Lója, J. Szigeti, and T. Cinkler, "Inter-domain routing in multiprovider optical networks: game theory and simulations," in Next Generation Internet Networks, 2005. IEEE, 2005, pp. 157-164.

[28] P. Marbach and R. Berry, "Downlink resource allocation and pricing for wireless networks," in INFOCOM 2002. Twenty-First Annual Joint Conference of the IEEE Computer and Communications Societies. Proceedings. IEEE, vol. 3. IEEE, 2002, pp. $1470-1479$. 
[29] G. Obando, A. Pantoja, and N. Quijano, "Building temperature control based on population dynamics," IEEE Transactions on Control Systems Technology, vol. 22, no. 1, pp. 404-412, 2014.

[30] G. D. Obando, N. Quijano, and A. D. Pantoja, "Population dynamics applied to building energy efficiency," in 2011 50th IEEE Conference on Decision and Control and European Control Conference (CDC-ECC). IEEE, 2011, pp. 4777-4782.

[31] L. Pavel, "A noncooperative game approach to osnr optimization in optical networks," IEEE Transactions on Automatic Control, vol. 51, no. 5, pp. 848-852, 2006.

[32] — Game theory for control of optical networks. Springer, 2012.

[33] E. Ramírez-Llanos and N. Quijano, "Analysis and control for the water distribution problem," CDC, pp. 4030 - 4035, 122010.

[34] — - "A population dynamics approach for the water distribution problem," International Journal of Control, vol. 83, no. 9, pp. 1947-1964, 2010.

[35] W. H. Sandholm, "Excess payoff dynamics and other well-behaved evolutionary dynamics," Journal of Economic Theory, vol. 124, no. 2, pp. 149-170, 2005.

[36] — - Population games and evolutionary dynamics. MIT press, 2010.

[37] C. U. Saraydar, N. B. Mandayam, and D. Goodman, "Pricing and power control in a multicell wireless data network," IEEE Journal on Selected Areas in Communications, vol. 19, no. 10, pp. 1883-1892, 2001.

[38] C. R. Schneiter, Y. Y. Haimes, D. Li, and J. H. Lambert, "Capacity reliability of water distribution networks and optimum rehabilitation decision making," Water Resources Research, vol. 32, no. 7, pp. 2271-2278, 1996.

[39] P. D. Taylor and L. B. Jonker, "Evolutionary stable strategies and game dynamics," Mathematical biosciences, vol. 40, no. 1, pp. 145-156, 1978. 
[40] J. W. Weibull, Evolutionary game theory. MIT press, 1997.

[41] H. Yaïche, R. R. Mazumdar, and C. Rosenberg, "A game theoretic framework for bandwidth allocation and pricing in broadband networks," IEEE/ACM Transactions on Networking (TON), vol. 8, no. 5, pp. 667-678, 2000.

[42] R. D. Yates, "A framework for uplink power control in cellular radio systems," IEEE Journal on Selected Areas in Communications, vol. 13, no. 7, pp. 1341-1347, 1995.

[43] A. C. Zecchin, A. R. Simpson, H. R. Maier, and J. B. Nixon, "Parametric study for an ant algorithm applied to water distribution system optimization," IEEE Transactions on Evolutionary Computation, vol. 9, no. 2, pp. 175-191, 2005. 